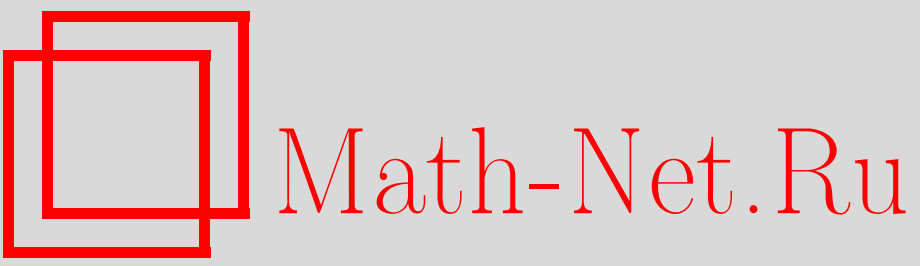

С. Б. Стечкин, А. Ю. Попов, Асимптотическое распределение простых чисел в среднем, УМH, 1996, том 51, выпуск 6, 21-88

DOI: https://doi.org/10.4213/rm1018

Использование Общероссийского математического портала Math-Net.Ru подразумевает, что вы прочитали и согласны с пользовательским соглашением

http://www . mathnet.ru/rus/agreement

Параметры загрузки:

IP : 3.95 .254 .165

26 апреля 2023 г., 02:15:52 


\section{АСИМПТОТИЧЕСКОЕ РАСПРЕДЕЛЕНИЕ ПРОСТЫХ ЧИСЕЛ В СРЕДНЕМ}

С.Б. СТЕчКИН, А. Ю. Попов

\section{СОДЕРЖАНИЕ}

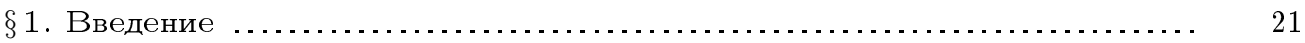

$\S 2$. Формулировки результатов ... . . . . . . . . . . . . . . . . . . . . . . . . . . 24

$\S 3$. Доказательства теорем 1-3 и их следствий ... . . . . . . . . . . . . . . . 36

§4. Доказательства теорем 4-14 и их следствий ................... . . 47

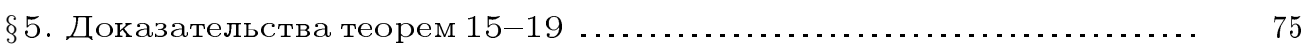

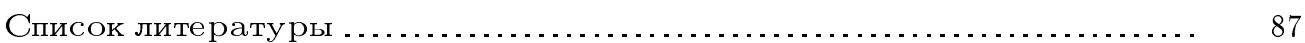

\section{$\S$ 1. Введение}

Пусть

$$
\begin{gathered}
\pi(x)=\sum_{p \leqslant x} 1, \quad \text { li } x=\int_{2}^{x}(\ln t)^{-1} d t, \\
\Lambda(n)= \begin{cases}\ln p, & n=p^{m}, \\
0 & \text { в противном случае },\end{cases} \\
\psi(x)=\sum_{n \leqslant x} \Lambda(n), \quad \psi_{0}(x)=\frac{\psi(x+0)+\psi(x-0)}{2} .
\end{gathered}
$$

Буквой $p$, как обычно, здесь обозначены простые числа.

Исследование поведения функции $\pi(x)$ является одной из центральных задач аналитической теории чисел. После того, как Адамар [1] и Валле-Пуссен [2] независимо доказали асимптотический закон

$$
\pi(x) \sim \frac{x}{\ln x} \sim \operatorname{li} x \quad(x \rightarrow+\infty),
$$

получение возможно лучших оценок сверху и снизу для модуля разности $P(x)=$ $\pi(x)-\operatorname{li} x$ стало одним из главных направлений исследований в теории распределения 
простых чисел. Отметим сразу же, что обычно изучают функцию $R(x)=\psi(x)-x$ или же $R_{0}(x)=\psi_{0}(x)-x$. Последнее оказьвается технически проше. Переход от $R(x)$ к $P(x)$ осушествляется с помошью известных соотношений [3, с. 124-125]

$$
\begin{aligned}
& \pi_{1}(x)=\sum_{2 \leqslant n \leqslant x} \frac{\Lambda(n)}{\ln n}=\frac{\psi(x)}{\ln x}+\int_{2}^{x} \frac{\psi(t) d t}{t \ln ^{2} t} \\
& \pi_{1}(x)=\pi(x)+\sum_{2 \leqslant m \leqslant \log _{2} x} \frac{\pi\left(x^{1 / m}\right)}{m},
\end{aligned}
$$

откуда немедленно вытекает, что

$$
P(x)=\frac{R(x)}{\ln x}+\int_{2}^{x} \frac{R(t) d t}{t \ln ^{2} t}-\frac{\sqrt{x}}{\ln x}+O\left(\frac{\sqrt{x}}{\ln ^{2} x}\right) .
$$

Равенство (1) показывает, что если $|R(x)|$ не слишком мал, то $|P(x)|$, грубо говоря, "в $\ln x$ раз меньше" чем $R(x)$.

В 1899 году Валле-Пуссен [4] доказал, что

$$
R(x)=O\left(x \exp \left\{-c_{1} \sqrt{\ln x}\right\}\right) .
$$

(Через $c_{1}, c_{2}, c_{3}$ и т. д. мы будем обозначать некоторые положительные постоянные.) Наилучшая по порядку из известных в настоящее время оценок сверху $|R(x)|$ (см. [5]-[9]) имеет вид

$$
R(x)=O\left(x \exp \left\{-c_{2}(\ln x)^{0.6}(\ln \ln x)^{-0.2}\right\}\right) \quad(x \geqslant 20) .
$$

Для ее доказательства потребовались очень глубокие и сложные теоремы об оценках тригонометрических сумм. Именно они и позволили получить неравенство

$$
|\zeta(\sigma+i t)| \leqslant c_{3}(\ln |t|)^{2 / 3}, \quad \sigma \geqslant 1-c_{4}(\ln |t|)^{-2 / 3}, \quad|t|>t_{0} .
$$

А уже из (3) с помощью методов, разработанных еще Валле-Пуссеном, можно вывести, что при $|t|>t_{0}$

$$
\zeta(\sigma+i t) \neq 0, \quad \sigma>1-c_{5}(\ln |t|)^{-2 / 3}(\ln \ln |t|)^{-1 / 3} .
$$

Последняя оценка дает (2). Подобные доказательства имеются в книге [10], а более полное изложение истории вопроса - в [8], см. также обзор [11].

Не желая умалять достоинства результата (2), отметим, что он носит “достаточный" характер и по сушеству лишь отражает имеюшиеся на сегодняшний день оценки сверху вещественных частей нулей дзета-функции Римана. Давно известно, что чем лучше оценка сверху для действительных частей нулей $\zeta(s)$, тем лучше и оценка сверху для $|R(x)|$ (а значит, ввиду (1) и для $|P(x)|$ ). Например, справедливо соотношение

$$
\limsup _{x \rightarrow+\infty} \frac{\ln |R(x)|}{\ln x}=\limsup _{x \rightarrow+\infty} \frac{\ln |P(x)|}{\ln x}=\Theta,
$$

где $\Theta=\sup \{\operatorname{Re} s \mid \zeta(s)=0\}$. 
Соотношение (4) показьвает, что в действительности наибольший рост функций $|R(x)|$ и $|P(x)|$ определяется величиной $\Theta$. Но, несмотря на усилия многих математиков, до сих пор про число $\Theta$ не известно ничего, кроме тривиального неравенства $1 / 2 \leqslant \Theta \leqslant 1$. У тверждение “ $\Theta=1 / 2$ ” составляет гипотезу Римана. С другой стороны, даже возможность $\Theta=1$ пока не исключена.

Обратим внимание на то обстоятельство, что в (4) нельзя заменить lim sup на lim. Это подтверждается теоремой Шмидта [12]

$$
\begin{aligned}
& R(x)=\Omega_{ \pm}\left(x^{\Theta-\varepsilon}\right), \quad(\forall \varepsilon>0) \\
& P(x)=\Omega_{ \pm}\left(x^{\Theta-\varepsilon}\right),
\end{aligned}
$$

и безусловными оценками Литтлвуда [13]

$$
\begin{aligned}
& R(x)=\Omega_{ \pm}(\sqrt{x} \ln \ln \ln x), \\
& P(x)=\Omega_{ \pm}\left(\sqrt{x}(\ln x)^{-1} \ln \ln \ln x\right) .
\end{aligned}
$$

Таким образом, функции $R(x)$ и $P(x)$ принимают “большие” по абсолютной величине как положительные, так и отрицательные значения, бесконечно много раз меняют знак и, следовательно, в окрестностях точек смены своего знака $R(x)$ и $P(x)$ по модулю "достаточно малы".

Имеем также [14]

$$
R(x)=O\left(x^{\Theta} \ln ^{2} x\right), \quad P(x)=O\left(x^{\Theta} \ln x\right) .
$$

Оценки (5)-(7) представляют из себя уточнение грубого соотношения (4).

Примечательно, что и после усреднения $R(x)$ функция $R_{1}(x)=\int_{0}^{x} R(u) d u$ остается колеблющейся. При этом упомянутые смены знака $R(x)$ не дают существенной интерференции. Имеем [15], [16, с. 120]

$$
\begin{aligned}
& R_{1}(x)=O\left(x^{1+\Theta}\right) \\
& R_{1}(x)=\Omega_{ \pm}\left(x^{1+\Theta-\varepsilon}\right) \quad(\forall \varepsilon>0) \\
& R_{1}(x)=\Omega_{ \pm}\left(x^{3 / 2}\right) .
\end{aligned}
$$

Правда, если справедлива гипотеза Римана, то между $O$-оценками и $\Omega$-оценками для $R_{1}(x)$ уже нет зазора, и аналог "феномена Литтлвуда" (неограниченность $R(x) / \sqrt{x}$ ) для функции $R_{1}(x)$ отсутствует: $R_{1}(x)=O\left(x^{3 / 2}\right)$. Но происходит это, в основном, не за счет компенсации в результате интегрирования положительных и отрицательных значений $R(x)$, а потому, что “литтлвудовские всплески" встречаются относительно редко. Это показывает теорема Крамера [17]

$$
\int_{1}^{x} R^{2}(u) u^{-1} d u=O(x) \quad(\text { если } \Theta=1 / 2)
$$

или же

$$
\left.\int_{1}^{x} R^{2}(u) d u \leqslant c_{6} x^{2} \quad \text { (если } \Theta=1 / 2\right) .
$$


Насколько нам известно, оценки (8)-(10) и асимптотика

$$
\Theta=1 / 2 \Longrightarrow \int_{1}^{x} R^{2}(u) u^{-2} d u \sim k \ln x, 0<k<+\infty(x \rightarrow+\infty),
$$

найденная в [18], составляют основные результаты о средних значениях функции $R(x)$, полученные ранее.

Наша работа посвящена изучению различного рода средних от функции $R(x)$. Выводятся оценки сверху и снизу для интегралов от функции $R(x)$ и степеней ее модуля. Соответствуюшие неравенства доказьваются и для $P(x)$. Основным нашим достижением мы считаем то, что нам удалось $\Omega$-оценки функций

$$
|P(u)|, \quad|R(u)|, \quad R^{+}(u)=\max (0, R(u)), \quad R^{-}(u)=-\min (0, R(u))
$$

заменить правильными по порядку оценками снизу от их интегралов по "не слишком длинным" отрезкам (от $x$ до $A x, A$ - постоянная). Доказаны как условные, так и безусловные (т.е. не зависящие от возможного значения $\Theta$ ) оценки. В качестве иллюстрации приведем безусловные оценки снизу.

Существуют положительные постоянные $x_{0}, A>1$ такие, что при всех $x>x_{0}$ справедливы неравенства

$$
\begin{array}{ll}
\int_{x}^{2 x}|R(u)| d u \geqslant \frac{x^{3 / 2}}{200}, & \int_{x}^{2 x}|P(u)| d u \geqslant \frac{x^{3 / 2}}{\ln x} \\
\int_{x}^{A x} R^{+}(u) d u \geqslant x^{3 / 2}, & \int_{x}^{A x} R^{-}(u) d u \geqslant x^{3 / 2} .
\end{array}
$$

Приведенный результат можно усилить, не производя сенсации, только за счет постоянного множителя при $x^{3 / 2}$ в правых частях неравенств. Ведь если бы даже для какой-нибудь последовательности $x_{n} \rightarrow+\infty$ было доказано, что

$$
\int_{x_{n}}^{A x_{n}}|R(u)| d u \geqslant \sqrt{c_{6} A^{3}} x^{3 / 2}
$$

$\left(c_{6}\right.$ - постоянная неравенства $\left.(10)\right)$, то гипотеза Римана была бы опровергнута. Действительно, в предположении справедливости гипотезы Римана из (10) находим

$$
\begin{aligned}
\int_{x_{n}}^{A x_{n}}|R(u)| d u & <\int_{1}^{A x_{n}}|R(u)| d u \leqslant\left(\int_{1}^{A x_{n}} R^{2}(u) d u\right)^{1 / 2}\left(\int_{1}^{A x_{n}} d u\right)^{1 / 2} \\
& \leqslant \sqrt{c_{6}\left(A x_{n}\right)^{2}} \sqrt{A x_{n}-1}<\left(A x_{n}\right)^{3 / 2} \sqrt{c_{6}} .
\end{aligned}
$$

Несколько слов о структуре работы. В $\S 2$ формулируются и обсуждаются полученные результаты. $\S \S 3-5$ посвящены их доказательствам.

\section{§ 2. Формулировки результатов}

ТЕОРема 1. Существует абсолютная әффективная постоянная $c_{7}$ такая, что при всех $b \geqslant 1$ и $x \geqslant 1$ справедливо неравенство

$$
\int_{1}^{x}|R(u)|^{b} d u \leqslant\left(c_{7} b\right)^{2 b} x^{1+b \Theta} .
$$


Обсудим содержание теоремы 1 . Пусть $b=1$. Тогда

$$
\int_{1}^{x}|R(u)| d u \leqslant c_{8} x^{1+\Theta}
$$

Из неравенства (2.2) вытекает, что результат от интегрирования $|R(u)|$ по отрезку $[1, x]$ не больше, чем от интегрирования $2 c_{8} u^{\Theta}$. То есть $R(u)$ “в среднем есть $O\left(u^{\Theta}\right)$ ", причем постоянные $c_{7}$ и $c_{8}$ - абсолютные, они не зависят от возможного значения $\Theta$. Более того, и $|R(u)|^{b}$ “в среднем есть $O\left(u^{b \Theta}\right)$ " при любом $b>1$. Теорема Крамера (10) содержится в теореме 1 при $\Theta=1 / 2, b=2$. Отметим, что при $\Theta=1 / 2$ ввиду (6) теорема 1 не следует из абсолютных оценок для функции $R$.

На случай $\Theta>1 / 2$ нам удалось асимптотически улучшить известное соотношение (7).

TeOpema 2. Если $\Theta>1 / 2$, mo

$$
|R(x)| \leqslant c_{9}(\Theta) x^{\Theta} .
$$

Постоянная $c_{9}$ здесь зависит от возможного значения $\Theta$. Если же $\Theta$ недостижимо, т.е. $\zeta(\Theta+i t) \neq 0$ при любом $t \in \mathbb{R}$, то

$$
R(x)=o\left(x^{\Theta}\right) \quad(x \rightarrow+\infty) .
$$

Нам не удалось доказать неравенство (3.2) с абсолютной постоянной $c_{9}$. Поэтому даже если гипотеза Римана не верна и мы бы знали о значении $\Theta$ только то, что $\Theta>1 / 2$, то и в этом случае теорема 1 не могла бы быть непосредственно вьведена из $(3.2)$, поскольку постоянная $c_{7}$ является абсолютной.

Заметим также, что если вдруг окажется (вопреки ожиданиям многих ученых!), что $\Theta=1$, то теоремы 1 и 2 станут тривиальньми и малосодержательными.

Приведем несколько следствий из сформулированных результатов. Положим

$$
\Delta(u)=R(u) u^{-\Theta} .
$$

СЛЕДСТВИЕ 1. При любъх $b \geqslant 1 u x \geqslant 1$

$$
x^{-1} \int_{1}^{x}|\Delta(u)|^{b} d u=O(1) .
$$

Постоянная в символе $O$ зависит только от $b$.

СлЕДСТВИЕ 2. Если $\lambda \leqslant\left(e c_{7}\right)^{-1}, m o$

$$
x^{-1} \int_{1}^{x} \exp \{\lambda \sqrt{|\Delta(u)|}\} d u=O(1) .
$$

Постоянная в символе $O$ абсолютная; она не зависит от возможного значения $\Theta$. Подчеркнем еше раз, что если $\Theta=1 / 2$, то согласно теореме Литтлвуда

$$
\Delta(x)=\Omega_{ \pm}(\ln \ln \ln x) .
$$

Таким образом, если верна гипотеза Римана, то оценки $R(u)$ в интегральных метриках принципиально отличаются от оценок максимума модуля этой функции. Те значения $u$, при которых $|R(u)| / \sqrt{u}$ велик, являются в некотором смысле исключениями из правила. Оценку меры больших значений $|R(u)| / \sqrt{u}$ дает 
СлеДСтвИЕ 3. Пусть справедлива гипотеза Римана. Обозначим

$$
\mathfrak{M}(V, x)=\operatorname{mepa}\{u \in[x, 2 x]|| R(u) \mid>\sqrt{u} V(u)\},
$$

где $V$ - некоторая неубьвающая функиия, стремящаяся $\kappa+\infty$ при $x \rightarrow+\infty$. Тогда

$$
\mathfrak{M}(V, x)=O\left(x \exp \left\{-c_{10} \sqrt{V(x)}\right\}\right)
$$

Заметим, что из следствия 3 несложно вывести известную теорему [19]: если $\Theta=1 / 2$, mo

$$
R(u)=O\left(\sqrt{u} \ln ^{2} u\right) .
$$

Усилить ее, однако, не удается. Это получилось бы, если бы мы могли в (1.2) заменить $\left(c_{7} b\right)^{2 b}$ на $(o(b))^{2 b}$.

СледСТВИЕ 4. Существует абсолютная постоянная $c_{11}$ такая, что при любыx $b \geqslant 1 u x \geqslant 4$

$$
\int_{2}^{x}|P(u)|^{b} d u \leqslant\left(c_{11} b\right)^{2 b} x^{1+b \Theta}(\ln x)^{-b} .
$$

СЛЕДСТВИЕ 5. Если $\Theta>1 / 2, m o$

$$
|P(x)| \leqslant c_{12}(\Theta) x^{\Theta} / \ln x .
$$

Если $\Theta$ недостижимо, то

$$
P(x)=o\left(x^{\Theta} / \ln x\right) \quad(x \rightarrow+\infty) .
$$

Из следствия 1, в свою очередь, вытекает

СЛЕДСТВИЕ 6. При любих $q \geqslant 1 u x \geqslant e$

$$
\int_{1}^{x}|\Delta(u)|^{q} u^{-1} d u=O(\ln x)
$$

Постоянная в $О$ зависит от $q$, но не от $\Theta$.

В частном случае $q=2$ в предположении справедливости гипотезы Римана Крамер [18] доказал утверждение более сильное, чем следствие 6:

$$
\int_{1}^{x} R^{2}(u) u^{-2} d u \sim k \ln x
$$

где $k=\sum_{\rho}\left(\nu_{\rho} /|\rho|\right)^{2}$, где $\nu_{\rho}-$ кратность нуля $\rho$, а $\sum_{\rho}$ понимается как сумма по всем различным нетривиальным нулям $\rho$ дзета-функции Римана. ${ }^{1}$

Нам удалось найти обобшение этого результата Крамера.

\footnotetext{
${ }^{1}$ Напомним, что нетривиальными называются все нули $\zeta(s)$, лежащие в полосе $0<\operatorname{Re} s<1$. Остальные нули являются простыми, имеют вид $\{-2 n \mid n \in \mathbb{N}\}$ и называются тривиальными. Все найденные к настоящему времени нетривиальные нули $\zeta(s)$ - простые, и до сих пор неизвестно, существуют ли у $\zeta(s)$ кратные нули.
} 
ТЕОРема 3. Если $b \in \mathbb{N}$, то существует предел

$$
\mathscr{B}(b)=\lim _{x \rightarrow+\infty}(\ln x)^{-1} \int_{1}^{x}(\Delta(u))^{b} u^{-1} d u
$$

әде

$$
\begin{gathered}
\mathscr{B}(b)=(-1)^{b} \sum_{\substack{\operatorname{Im}\left(\sum_{k=1}^{b} \rho^{(k)}\right)=0 \\
\operatorname{Re} \rho^{(k)}=\Theta, 1 \leqslant k \leqslant b}} \prod_{k=1}^{b}\left(\rho^{(k)}\right)^{-1} . \\
\end{gathered}
$$

Суммирование в ряде $(7.2)$ ведется по всем наборам $\left(\rho^{(1)}, \ldots, \rho^{(b)}\right)$ нетривиальных нулей $\zeta(s)$, для которых $\operatorname{Im}\left(\sum_{k=1}^{b} \rho^{(k)}\right)=0$, a $\operatorname{Re} \rho^{(k)}=\Theta, 1 \leqslant k \leqslant b$. Каждый нуль $\rho^{(k)}$ встречается в качестве $k$-й компоненты набора столько раз, какова его кратность. Доказано, что ряд (7.2) абсолютно сходится. Если $\Theta$ недостижимо, то сумма (7.2) пуста и $\mathscr{B}(b)=0$.

В случае $b=2$ имеем

$$
\begin{gathered}
\mathscr{B}(2)=\sum_{\substack{\operatorname{Im}\left(\rho^{(1)}+\rho^{(2)}\right)=0 \\
\operatorname{Re} \rho^{(1)}=\operatorname{Re} \rho^{(2)}=\Theta}} \frac{1}{\rho^{(1)} \rho^{(2)}}=\sum_{\substack{\rho-\text { различны } \\
\operatorname{Re} \rho=\Theta}} \nu_{\rho}^{2}|\rho|^{-2}, \\
\mathscr{B}(2)=\lim _{x \rightarrow+\infty}(\ln x)^{-1} \int_{1}^{x} R^{2}(u) u^{-1-2 \Theta} d u .
\end{gathered}
$$

Тем самым получается более сильная теорема, чем упомянутая теорема Крамера, поскольку здесь не делается никаких предположений о величине $\Theta$.

Заметим, что $\mathscr{B}(1)=0$. Сумма пуста, так как $\operatorname{Im} \rho \neq 0$. Авторам не известно, пуста или нет сумма $\mathscr{B}(2 r+1), r \in \mathbb{N}$, даже для достижимого $\Theta$. С другой стороны, из симметричности нулей $\zeta(s)$ относительно действительной оси вытекает, что если $\Theta$ достижимо, то $\mathscr{B}(2 r)>0$ при любых $r \in \mathbb{N}$.

Перейдем к оценкам снизу интегралов от $|R(x)|, R^{+}(x), R^{-}(x)$. Напомним, что

$$
R^{+}(x)=\max (0, R(x)), \quad R^{-}(x)=-\min (0, R(x)) .
$$

Центральньм результатом этого раздела являются формулируемые ниже теоремы 4 и 5.

ТЕОрема 4. Существует, вообще говоря неэффективная, постоянная $c_{13}$ такая, что при любом $x>c_{13}$ выполняется неравенство

$$
\int_{x}^{2 x}|R(u)| d u>\frac{x^{3 / 2}}{200}
$$


ТЕОРема 5. Существуют, вообще говоря неэффективные, положительные постоянные $c_{14}$ и A такие, что при любом $x>c_{14}$ выполняются неравенства

$$
\begin{aligned}
& \int_{x}^{A x} R^{+}(x) d x>x^{3 / 2}, \\
& \int_{x}^{A x} R^{-}(x) d x>x^{3 / 2} .
\end{aligned}
$$

В случае $\Theta=1 / 2$ утверждение теоремы 5 справедливо с $A=212$.

Как отмечалось во введении, если справедлива гипотеза Римана, то неравенства теорем 4 и 5 можно усилить только за счет постоянных в правых частях. Особенно интересно на наш взгляд то, что удалось дать оценки снизу в среднем для $|R|, R^{+}$, $R^{-}$на "не слишком длинном" отрезке. Мы полагаем, что следствия 7 и 8 являются новыми (см., например, [20]).

СлЕДСТВИЕ 7 . При $x>c_{13}$ на отрезке $[x, 2 x]$ найдется число и такое, что

$$
|R(u)|>\frac{\sqrt{u}}{245}
$$

СЛЕДСТВИЕ 8. Если А и $c_{14}>0$ - постоянные теоремы 5, то при всех $x>c_{14}$ на отрезке $[x, A x]$ найдутся точки $u_{1}$ и $u_{2}$, в которых выполняются неравенства

$$
R\left(u_{1}\right)>A^{-3 / 2} \sqrt{u_{1}}, \quad R\left(u_{2}\right)<-A^{-3 / 2} \sqrt{u_{2}} .
$$

К достоинствам теорем 4 и 5 , следствий 7 и 8 можно отнести их безусловность. Они верны при любом возможном значении $\Theta$. Недостатком является неэффективность этих результатов. Мы не можем указать значения постоянных $A, c_{13}$ и $c_{14}$. Дело в том, что теорема 4 , например, вытекает из формулируемых ниже теорем 6 и 7 . В теореме 6 рассматривается случай $\Theta=1 / 2$, и в ней всё эффективно. Теорема 7 доказьвается в предположении, что гипотеза Римана неверна. Для того чтобы наш метод ее доказательства дал эффективную постоянную $c_{13}$, нужно обладать достаточно большой информацией о нулях $\zeta$. В частности, знать оценку сверху ординаты какого-нибудь нуля $\zeta(s)$ с вешественной частью, большей $1 / 2$. Такой ход рассуждений и приводит к неэффективности безусловной теоремы, несмотря на то, что при $\Theta>1 / 2$ оценки снизу интегралов от $|R|, R^{+}, R^{-}$асимптотически лучше, чем при справедливости гипотезы Римана. Ситуация с теоремой 5 почти аналогична.

Теорема 6. Если справедлива гипотеза Римана, то существует әффективная постоянная $c_{15}$ такая, что при любых $x>c_{15}$ выполняется неравенство

$$
\int_{x}^{2 x}|R(u)| d u>\frac{x^{3 / 2}}{200}
$$

Из теоремы 6 и теоремы 1 немедленно вытекает 
СлЕДСТВИЕ 9. Если $\Theta=1 / 2$, то при $a \geqslant 2$

$$
\int_{x}^{a x}|R(u)| d u \underset{a}{\asymp} x^{3 / 2} \quad(x \rightarrow+\infty) .
$$

Весьма вероятно, что утверждение (8.2) справедливо при любом $a>1$, но доказать это мы не умеем.

Теорема 7. Если $\Theta>1 / 2$, то для любих $\varepsilon>0$ u $а>1$ cyществует постоянная $x_{0}$, зависящая от $\varepsilon$ и а, такая, что при всех $x>x_{0}$ справедливо неравенство

$$
\int_{x}^{a x}|R(u)| d u>x^{1+\Theta-\varepsilon}
$$

Если $\Theta>1 / 2$ достижимо (т.е. при некотором $\widetilde{\gamma} \in \mathbb{R}$ имеем $\zeta(\Theta+i \widetilde{\gamma})=0$ ), то теорему 7 можно несколько уточнить.

ТЕОРема 8. Если $\Theta>1 / 2$ является достижимим, то для любого $а>1$ существует положительная постоянная $c_{16}$, зависящая от а, такая, что при всех $x \geqslant 1$

$$
\int_{x}^{a x}|R(u)| d u \geqslant c_{16} x^{1+\Theta}
$$

Постоянные $x_{0}$ в теореме 7 и $c_{16}$ в теореме 8 зависят от возможного значения $\Theta$ и величин, указанных в формулировках этих теорем, неэффективно. Чтобы сосчитать эти постоянные в рамках наших методов требуется обладать достаточно обширной информацией о распределении нулей $\zeta(s)$ в полуплоскости $\operatorname{Re} s>1 / 2$ (если они вообше там имеются). Заметим, что если бы постоянная $x_{0}$ в теореме 7 была эффективной, то мы получили бы алгоритм вычисления $\Theta$ с любой наперед заданной точностью, на что в настоящее время мало надежды.

Сравнивая оценки теорем 7 и 8 с (2.2), мы видим, что (9.2) может быть улучшено только за счет $\varepsilon$, а $(10.2)$ - за счет $c_{16}$. В этом смысле результаты теорем 7 и 8 по порядку точны.

Теорема 9. Если справедлива гипотеза Римана, то при всех $x>2 \cdot 10^{9}$ въ-полняются неравенства

$$
\int_{x}^{10 x} R^{+}(u) d u \geqslant 0.01 x^{3 / 2}, \quad \int_{x}^{10 x} R^{-}(u) d u \geqslant 0.01 x^{3 / 2} .
$$

ТЕОрема 10. Предположим, что $\Theta>1 / 2$ недостижимо. Тогда для любъх $\varepsilon>0$ и $а>1$ при всех $x>x_{1}(\varepsilon, a)$ выполняются неравенства

$$
\int_{x}^{a x} R^{+}(u) d u>x^{1+\Theta-\varepsilon}, \quad \int_{x}^{a x} R^{-}(u) d u>x^{1+\Theta-\varepsilon} .
$$


ТЕорема 11. Предположим, что $\Theta>1 / 2$ достижимо. Обозначим

$$
k_{1}=\sum_{\substack{\text { Re } \rho=\Theta \\ \rho-\text { различны }}}|\operatorname{Im} \rho|^{-1}, \quad A_{1}=\exp \left\{2 \pi k_{1}\right\} .
$$

Тогда существуют положительные постоянные $c_{17}$ и $c_{18}$ такие, что при всех $x>c_{18}$ выполняются неравенства

$$
\int_{x}^{A_{1} x} R^{+}(u) d u>c_{19} x^{1+\Theta}, \quad \int_{x}^{A_{1} x} R^{-}(u) d u>c_{19} x^{1+\Theta} .
$$

Здесь и далее под символом

$$
\sum_{\rho \in E} f(\rho)
$$

где $E \subset \mathbb{C}, f: E \rightarrow \mathbb{C}$, понимается сумма значений функции $f$ в нетривиальных нулях $\zeta(s)$, принадлежащих множеству $E$. Если не оговорено противное, то считается, что каждый нуль в сумме (12.2) встречается столько раз, какова его кратность. Если ряд не является абсолютно сходящимся, то под его суммой понимается предел конечных сумм

$$
\lim _{T \rightarrow+\infty} \sum_{\substack{\rho \in E \\|\operatorname{Im} \rho| \leqslant T}} f(\rho) .
$$

У нас будут встречаться только такие ряды (12.2), которые будут сходиться в смысле сушествования предела (13.2) или даже абсолютно сходиться.

Ряд, сумму которого мы обозначили через $k_{1}$, абсолютно сходится, если $\Theta>1 / 2$. Это вытекает из простейшей плотностной теоремы [21, с 237].

ТеОрема 12. При любом $x>c_{19}$ справедливо неравенство

$$
\int_{x}^{2 x}|P(u)| d u>x^{3 / 2}(\ln x)^{-1}
$$

Если $\Theta=1 / 2$, то постоянная с 19 әффективна. Если $\Theta>1 / 2$, то какови би ни бълли $\varepsilon>0$ и $а>>1$ при $x>c_{20}(a, \varepsilon)$ имеем

$$
\int_{x}^{a x}|P(u)| d u>x^{1+\Theta-\varepsilon}
$$

Если жее $\Theta>1 / 2$ является достижимым, то при любом $а>1$ и $x>c_{21}(a)$ имеем

$$
\int_{x}^{a x}|P(u)| d u>c_{22}(a) x^{1+\Theta}(\ln x)^{-1}
$$

Невозможность принципиального улучшения теоремы 12 вытекает из следствия 4 $(b=1)$. Как и в теореме 4 , постоянная $c_{19}$ в безусловной оценке $(14.2)$ является неэффективной. 
Случай $\Theta=1 / 2$ разобран отдельно в теоремах 13 и 14 . Если справедлива гипотеза Римана, то в некотором смысле "чаще всего" $P(x)<0$. Следуюшая теорема представляет из себя уточнение результата, имеюшегося на с. 138-139 в [16]. Положим

$$
\begin{gathered}
\mathscr{P}(x)=x^{-3 / 2} \ln x \int_{2}^{x} P(u) d u, \\
\omega=\sum_{\rho} \frac{1}{\sqrt{\gamma^{4}+2,5 \gamma^{2}+9 / 16}}, \quad \gamma=\operatorname{Im} \rho .
\end{gathered}
$$

Если верна гипотеза Римана, то

$$
\omega=\sum_{\rho} \frac{1}{|\rho(\rho+1)|}<\sum_{\rho} \frac{1}{|\rho|^{2}}<0.0456 .
$$

(См. ниже (20.4) в доказательстве теоремы 6.)

Теорема 13. Если справедлива гипотеза Римана, то выполняются оценки

$\Pi$ пи этом $\underline{L} \neq \bar{L}$.

$$
\begin{aligned}
& \bar{L}=\limsup _{x \rightarrow+\infty} \mathscr{P}(x) \leqslant-\frac{2}{3}+\omega, \\
& \underline{L}=\liminf _{x \rightarrow+\infty} \mathscr{P}(x) \geqslant-\frac{2}{3}-\omega .
\end{aligned}
$$

СлеДСТвИЕ 10. Если верна гипотеза Римана, то при всех $x>c_{23}$ справедливиц неравенства

$$
-0.714 x^{3 / 2}(\ln x)^{-1}<\int_{1}^{x} P(u) d u<-0.62 x^{3 / 2}(\ln x)^{-1} .
$$

При $\Theta=1 / 2$ отрицательные значения функции $P$ превалируют над положительными и на отрезках вида $[x, a x]$, где $a$ "не слишком близко" к 1 . Наоборот, при $a$, близких к 1, бесконечно часто наблюдается противоположный эффект.

Теорема 14. Предположим, что справедлива гипотеза Римана. Тогда

1) при любом $a>1$ существует әффективная постоянная $c_{24}=c_{24}(a)$ такая, что при всех $x>c_{24}$ выполнено неравенство

$$
\int_{x}^{a x} P(u) d u<-\frac{2}{3} \frac{x^{3 / 2}}{\ln x}\left(a^{3 / 2}-1\right) K(a)+O\left(\frac{x^{3 / 2}}{\ln ^{2} x}\right),
$$

$2 \partial e$

$$
K(a)=1-\frac{3 \omega}{2} \frac{\left(a^{3 / 2}+1\right)}{\left(a^{3 / 2}-1\right)} ;
$$

2) существует эффективная постоянная $c_{25}$ такая, что при всех $X \geqslant 3$ и $\alpha \in\left(0, c_{25}\right)$ на отрезке $\left[X, X^{B}\right], B=\exp \left\{\alpha^{-1} \ln ^{3} 1 / \alpha\right\}$, найдется точка $\xi$, для которой выполняется неравенство

$$
\int_{\xi}^{\xi+\alpha \xi} P(u) d u>\frac{\xi^{3 / 2} \alpha \ln (1 / \alpha)}{6 \ln \xi} ;
$$

3) существует постоянная $A_{2}>1$ такая, что при всех $x>x_{2}$ имеет место неравенство

$$
\int_{x}^{A_{2} x} P^{+}(u) d u>\frac{x^{3 / 2}}{\ln x}
$$


СлЕДСТВИЕ 11. Если справедлива гипотеза Римана, то при достаточно больиих $x$ имеем

$$
\begin{gathered}
\int_{x}^{1,2 x} P(u) d u<-0.1 x^{3 / 2}(\ln x)^{-1}, \\
\int_{x}^{2 x} P(u) d u<-x^{3 / 2}(\ln x)^{-1}, \\
\int_{x}^{2 x}|P(u)| d u \geqslant \int_{x}^{2 x} P^{-}(u) d u>x^{3 / 2}(\ln x)^{-1},
\end{gathered}
$$

а при любом фиксированном $а>1$

$$
\int_{x}^{a x} P^{+}(u) d u=\Omega\left(x^{3 / 2}(\ln x)^{-1}\right)
$$

Неравенства следствия 11 по порядку неулучшаемы (см. следствие 4 при $b=1$ ). Нам не известно, можно ли к безусловной оценке (14.2) добавить оценки снизу

$$
\int_{x}^{A_{2} x} P^{+}(u) d u>x^{3 / 2}(\ln x)^{-1}, \quad \int_{x}^{A_{2} x} P^{-}(u) d u>x^{3 / 2}(\ln x)^{-1}
$$

хотя бы при каком-нибудь, пусть даже очень большом, но постоянном значении $A_{2}$. Таким образом, мы не смогли перенести на функцию $P$ результат теоремы 5 . В теореме 14 мы доказали это в предположении справедливости гипотезы Римана с постоянной $A_{2}=2$ для $P^{-}$(см. $\left.(21.2)\right)$ и с некоторой неэффективной постоянной $A_{2}$ для $P^{+}$. По-видимому, эта задача не является легкой в случае, если $\Theta$ недостижимо. Если $\Theta>1 / 2$ достижимо, то из теоремы 11 и леммы 1 (см. ниже) почти тривиально получается

СлЕДСТВИЕ 12. Если $\Theta>1 / 2$ достижимо, то при $x>c_{26}$

$$
\begin{aligned}
& \int_{x}^{A_{1} x} P^{+}(u) d u>c_{27} x^{1+\Theta}(\ln x)^{-1} \\
& \int_{x}^{A_{1} x} P^{-}(u) d u>c_{27} x^{1+\Theta}(\ln x)^{-1}
\end{aligned}
$$

Постоянная $A_{1}$ определена в теореме 11.

Следуюшая часть наших результатов посвящена обобщению на средние для $R(x)$ по “коротким отрезкам" теоремы Литтлвуда о бесконечно больших в сравнении с $\sqrt{x}$ при $x \rightarrow+\infty$ значениях $R(x)$. В 1914 году Литтлвуд доказал, что

$$
R(x)=\Omega_{ \pm}(\sqrt{x} \ln \ln \ln x) .
$$

Эта безусловная $\Omega_{ \pm}$-оценка функции $R$ остается лучшей по порядку и на сегодняшний денњ. 
Пусть $H(x)$ - некоторая функция, положительная на $(1,+\infty)$. Рассмотрим среднее значение для $R$ на отрезке $[x, x+H(x)]$ :

$$
R_{1}(x, H(x))=\frac{1}{H(x)} \int_{x}^{x+H(x)} R(u) d u .
$$

Из (10) при $\Theta=1 / 2$ (см. также теорему 1 при $b=1)$ немедленно вытекает, что если $H(x) \geqslant \alpha x, \alpha>0$, то

$$
\int_{x}^{x+H(x)}|R(u)| d u=O\left((x+H(x))^{3 / 2}\right)
$$

и, тем более,

$$
R_{1}(x, H(x))=O(\sqrt{x+H(x)}) .
$$

Таким образом, если справедлива гипотеза Римана, то при усреднении функции $R$ по "не очень короткому" отрезку феномен Литтлвуда пропадает, и $R(u)$ "в среднем есть $O(\sqrt{u})$ ".

В связи со сказанным возникает следующая задача. Требуется выяснить, для каких $H(x)=o(x)(x \rightarrow+\infty)$ функция $x^{-1 / 2} R_{1}(x, H(x))$ неограничена или даже

$$
R_{1}(x, H(x))=\Omega_{ \pm}(\sqrt{x} \ln \ln \ln x) .
$$

Из (24.2) очевидным образом вытекает (23.2). Поэтому утверждения типа (24.2) можно рассматривать как обобшения упомянутого результата Литтлвуда. Отметим сразу же, что для

$$
H(x)=o(\sqrt{x} \ln \ln \ln x) \quad(x \rightarrow \infty)
$$

соотношение (24.2) тривиально следует из (23.2). Действительно, исходя из равенства

$$
R(x+y)-R(x)=-y+\sum_{x<n \leqslant x+y} \Lambda(n), \quad y>0
$$

и равномерной по $x$ оценки [22]

$$
\pi(x+y)-\pi(x)=O\left(\frac{y+2}{\ln (y+2)}\right), \quad y>0
$$

при $1 \leqslant y \leqslant x$ получаем

$$
R(x+y)-R(x)=O\left(\frac{(y+2) \ln x}{\ln (y+2)}\right)
$$


и, следовательно,

$$
\begin{aligned}
R_{1}(x, H(x)) & =R(x)+\frac{1}{H(x)} \int_{0}^{H(x)}(R(x+y)-R(x)) d y \\
& =R(x)+\frac{1}{H(x)} \int_{0}^{H(x)} O\left(\frac{(y+2) \ln x}{\ln (y+2)}\right) d y \\
& = \begin{cases}R(x)+O\left(\frac{H(x) \ln x}{\ln H(x)}\right), & H(x) \geqslant 3, \\
R(x)+O(\ln x), & 0<H(x)<3 .\end{cases}
\end{aligned}
$$

Из (16.2), (25.2) и (23.2) следует (24.2).

Если же функция $x^{-1 / 2-\delta} H(x)$ неограничена на $(1,+\infty)$ при некотором $\delta>0$, то вряд ли (24.2) можно вывести из (23.2) на основе таких же простых соображений. Для достаточно широкого класса функций $H(x)=o(x)(x \rightarrow+\infty)$ нам удалось решить поставленную выше задачу. В теоремах 15-19 будет предполагаться, что задано $x_{0}>0$, $H(x) \geqslant 1-$ произвольная действительнозначная функция на луче $\left[x_{0},+\infty\right)$.

ТеОРема 15. Если при некотором $\delta>0$ имеем

$$
H(x)=O\left(x^{1-\delta}\right)
$$

mo

$$
R_{1}(x, H(x))=\Omega_{ \pm}(\sqrt{x} \ln \ln \ln x) .
$$

Отметим, что теорема 15 является безусловной. Для функций $H(x)=o(x)$, растущих быстрее, чем (27.2), мы смогли обобшить результат Литтлвуда только лишш для достижимого $\Theta$, в частности, в предположении справедливости гипотезы Римана.

Teopema 16.

1) $\operatorname{Ecлu} \Theta=1 / 2 u$

$$
H(x)=O\left(x(\ln \ln x)^{-1}\right),
$$

mo

$$
R_{1}(x, H(x))=\Omega_{ \pm}(\sqrt{x} \ln \ln \ln x) .
$$

2) Если $\Theta>1 / 2$ достижимо и $H(x)=o(x)(x \rightarrow+\infty)$, то

$$
R_{1}(x, H(x))=\Omega_{ \pm}\left(x^{\Theta}\right)
$$

и, следовательно, тем более, верна оценка (29.2).

В предположении справедливости гипотезы Римана на функции, растущие быстрее, чем в (28.2), пришлось наложить дополнительные условия регулярности. 
ТЕОРема 17. Пусть функиия $H$ имеет на $\left(x_{0},+\infty\right)$ непрерывную неотрицательную производную. Обозначим для краткости $V(x)=x / H(x)$. Пусть затем

$$
\begin{gathered}
V(x) \leqslant \ln \ln x, \quad V(x) \uparrow+\infty, \\
\frac{d}{d x}\left(\frac{1}{V(x)}\right)=O\left(\frac{1}{x \ln x}\right), \\
V\left(x^{\ln x}\right) \leqslant(V(x))^{\lambda},
\end{gathered}
$$

где $\lambda$ - некоторая постоянная. Тогда если $\Theta=1 / 2$, то

$$
R_{1}(x, H(x))=\Omega_{ \pm}(\sqrt{x} \ln V(x))
$$

Условия (30.2) накладьвают некоторые ограничения на регулярность роста функции $H$, подчиненной условию $x(\ln \ln x)^{-1} \leqslant H(x)=o(x)(x \rightarrow+\infty)$. Ясно, что они выполняются для всех “достаточно медленно" и “достаточно правильно" растущих функций $V(x)$. В частности, условиями (30.2) удовлетворяют все функции $H(x)=x(\ln \ln x)^{\alpha},-1 \leqslant \alpha<0, H(x)=x(\ln \ln \ln x)^{\alpha}, \alpha<0$, и т. д.

Теорема 18. Пусть справедлива гипотеза Римана. Тогда равномерно по $x \geqslant 10,0<v<1 / 3$ справедлива оценка

$$
\int_{x}^{x+v x} R^{2}(u) d u=O\left(v x^{2} \ln ^{4} v\right) .
$$

В частности, если $H(x)=o(x)(x \rightarrow+\infty)$, то

$$
R_{1}(x, H(x))=O\left(\sqrt{x} \ln ^{2}(x / H(x))\right) .
$$

Постоянные в символах $O$ - абсолютные и әффективные.

Из (29.2), (31.2) и (33.2) видно, что даже при $\Theta=1 / 2$ и любых допустимых $H(x)=o(x)(x \rightarrow+\infty)$ сохраняется зазор между $\Omega$-оценкой $R_{1}(x, H(x))$ и оценкой сверху этой функции. Правда, чем медленнее убьвает $H(x) / x$, тем этот зазор меньше.

Если не задаваться целью устанавливать количественные $\Omega_{ \pm}$-оценки $(29.2)$ и (31.2), то в предположении $\Theta=1 / 2$ можно доказать теорему более простую, чем теоремы 15-17, не накладывая при этом на функцию $H(x)$ сушественных ограничений.

Теорема 19. Предположим, что $\Theta=1 / 2$,

$$
1 \leqslant H(x)=o(x) \quad(x \rightarrow+\infty)
$$

Тогда функция $x^{-1 / 2} R_{1}(x, H(x))$ неограничена как сверху, так и снизу.

Теоремы 15-18 автоматически переносятся с $R_{1}(x, H(x))$ на

$$
P_{1}(x, H(x))=\frac{1}{H(x)} \int_{x}^{x+H(x)} P(u) d u .
$$


При замене в $(27.2),(29.2)$ и (31.2) $R_{1}$ на $P_{1}$ требуется правые части этих соотношений разделить на $\ln x$. Если $\Theta=1 / 2,1 \leqslant H(x)=o(x)(x \rightarrow+\infty)$, то функция $x^{-1 / 2} P_{1}(x, H(x)) \ln x$ неограничена как сверху, так и снизу.

Некоторые из результатов, сформулированных в этом параграфе, были анонсированы нами в [23]-[25].

В заключение $\S 2$ скажем несколько слов о методах исследования, используемых в этой работе, и поясним, за счет чего нам удалось получить новые результаты. Для $R_{0}(x)$ (определение см. в начале статьи) известна формула, открытая Риманом, а потом доработанная Мангольдтом, Э. Ландау и другими:

$$
-R_{0}(x)=\sum_{\rho} \frac{x^{\rho}}{\rho}+\ln 2 \pi+\frac{1}{2} \ln \left(1-\frac{1}{x^{2}}\right) .
$$

Ряд $\sum_{\rho} x^{\rho} / \rho$ по нетривиальным нулям $\zeta(s)$ сходится в смысле (13.2). Нетривиальные нули $\zeta(s)$ расположены симметрично относительно действительной оси, а для функции $\mathscr{N}(T)$, равной количеству нулей $\zeta(s)$ в прямоугольнике $\{0<\operatorname{Re} s<1$, $0<\operatorname{Im} s \leqslant T\}$, справедлива асимптотика [10, с. 44] с эффективной постоянной в символе $O$

$$
\mathscr{N}(T)=\frac{T \ln T}{2 \pi}-\frac{1+\ln 2 \pi}{2 \pi} T+O(\ln T)
$$

Обозначим

$$
\mathscr{F}(x)=\sum_{\rho} \frac{x^{\rho}}{\rho}
$$

Из (35.2) (см. также [16, с. 105-106]) очевидным образом вытекает, что

$$
\begin{array}{rlrl}
R_{0}(x) & =-\mathscr{F}(x)+O(1), & & x \geqslant 2, \\
R_{0}(x) & =-\mathscr{F}(x)+O(\ln (x-1)), & 1<x<2, \\
R(x) & =-\mathscr{F}(x)+O(\ln x), & & x \geqslant 2 .
\end{array}
$$

Поэтому совершенно ясно, что теоремы 1-11 и 15-19 достаточно доказать для $\mathscr{F}(x)$ вместо $R(x)$. Функция $\mathscr{F}\left(e^{t}\right)=\sum_{\rho} \exp (\rho t) / \rho$ представляет из себя ряд экспонент, показатели которых - нетривиальные нули дзета-функции Римана.

Мы не доказали ничего нового о нулях $\zeta(s)$, но зато разработали ряд технических приемов для оценок интегралов от степеней модуля, положительных и отрицательных частей рядов экспонент такого рода. Это и позволило нам получить все основные теоремы этой работы.

\section{§ 3. Доказательства теорем 1-3 и их следствий}

ДокАЗАТЕЛЬСтво теоРемы 1. Заметим сперва, что неравенство (1.2) достаточно установить лишь для четных целых $b$. Тогда для остальных $b$ оно окажется верным 
с постоянной $2 c_{7}$ вместо $c_{7}$. Действительно, если $b / 2 \notin \mathbb{N}$, то возьмем в качестве $m$ наименьшее четное число, превосходяшее $b$. Если

$$
\int_{1}^{x}(R(u))^{m} d u \leqslant\left(c_{28} m\right)^{2 m} x^{1+m \Theta}
$$

то, обозначив $q=m / b, 1 / q^{\prime}=1-1 / q$, используя неравенство Гёльдера, получим

$$
\begin{aligned}
\int_{1}^{x}|R(u)|^{b} d u & \leqslant\left(\int_{1}^{x}(R(u))^{m} d u\right)^{1 / q}\left(\int_{1}^{x} d u\right)^{1 / q^{\prime}} \\
& \leqslant\left(c_{28} m\right)^{2 b} x^{1 / q+b \Theta}(x-1)^{1 / q^{\prime}} \\
& \leqslant\left(c_{28} m\right)^{2 b} x^{1+b \Theta} \\
& \leqslant\left(2 c_{28} b\right)^{2 b} x^{1+b \Theta}
\end{aligned}
$$

а это и требовалось установить.

Ввиду сказанного, а также соотношений (38.2) заключаем, что для доказательства теоремы 1 достаточно при $b=2 r, r \in \mathbb{N}$, проверить справедливость неравенства

$$
\int_{1}^{x}(\mathscr{F}(u))^{b} d u \leqslant\left(c_{29} b\right)^{2 b} x^{1+b \Theta} .
$$

Как известно [3], последовательность частичных сумм $\sum_{|\operatorname{Im} \rho| \leqslant T} x^{\rho} / \rho$ ряда $(37.2)$ сходится к $\mathscr{F}(x)$ при $T \rightarrow+\infty$ (для удобства будем считать, что $T \in \mathbb{N}$ ) в каждой точке $x \in(1,+\infty)$. Следовательно, при любом $u>1$

$$
\lim _{T \rightarrow \infty} S(T, b, u)=(\mathscr{F}(u))^{b}
$$

где

$$
S(T, b, u)=\sum_{\substack{\rho_{1} \\\left|\operatorname{Im} \rho_{j}\right| \leqslant T}} \cdots \sum_{\rho_{b}} \frac{u^{\rho_{1}+\cdots+\rho_{b}}}{\rho_{1} \cdot \ldots \cdot \rho_{b}}
$$

Напомним лемму Фату [26, с. 40]. Пусть на промежутке $\omega \subset \mathbb{R}$ задана последовательность неотрицательных функций $f_{T} \in L(\omega)(\forall T \in \mathbb{N})$. Пусть затем известно, что

$$
\sup _{T \in \mathbb{N}} \int_{\omega} f_{T}(u) d u=M<+\infty
$$

и при почти всех $u \in \omega$

$$
\exists \lim _{T \rightarrow+\infty} f_{T}(u)=f(u) \in \mathbb{R}
$$

Тогда $f \in L(\omega)$ и $0 \leqslant \int_{\omega} f(u) d u \leqslant M$.

Функции $S(T, 1, u)$ действительнозначны при $u \in \mathbb{R}$, поскольку нули $\zeta(s)$ расположены симметрично относительно действительной оси. Поэтому последовательность 
функций $S(T, b, u)=(S(T, 1, u))^{b}$ при $b=2 r, r \in \mathbb{N}$, неотрицательна. Интегралы от $S(T, b, u)$ по отрезку $[1, x]$ легко вычисляются:

$$
\begin{aligned}
& \int_{1}^{x} S(T, b, u) d u=\sum_{\rho_{1}} \cdots \sum_{\rho_{b}} \frac{x^{1+\rho_{1}+\cdots+\rho_{b}}-1}{\left(1+\rho_{1}+\cdots+\rho_{b}\right) \rho_{1} \cdot \cdots \cdot \rho_{b}} . \\
& \left|\operatorname{Im} \rho_{j}\right| \leqslant T
\end{aligned}
$$

Поэтому, если мы покажем, что

$$
M_{1}(b)=\sum_{\rho_{1}} \cdots \sum_{\rho_{b}}\left(\left|1+\rho_{1}+\cdots+\rho_{b}\right| \prod_{j=1}^{b}\left|\rho_{j}\right|\right)^{-1} \leqslant\left(c_{30} b\right)^{2 b},
$$

то из (3.3), (4.3), учитывая, что $\operatorname{Re} \rho_{j} \leqslant \Theta$, получим

$$
M(b, x)=\sup _{T} \int_{1}^{x} S(T, b, u) d u \leqslant 2 x^{1+b \Theta} M_{1}(b)<\left(2 c_{30} b\right)^{2 b} x^{1+b \Theta} .
$$

А это вместе с (2.3) и леммой Фату приведет нас к (1.3).

Итак, осталось доказать неравенство (4.3). Имеем:

$$
M_{1}(b)=\sum_{\bar{n}=\left(n_{1}, \ldots, n_{b}\right) \in \mathbb{Z}^{b}} A(\bar{n}),
$$

где

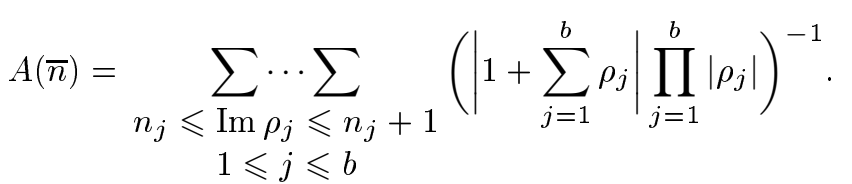

Оценим $A(\bar{n})$ сверху. Обозначим $\nu(\bar{n})=\sum_{j=1}^{b} n_{j}$. Поскольку $\operatorname{Re} \rho>0$, то в сумме $A(\bar{n})$ стоят нули, для которых

$$
\operatorname{Re}\left(\sum_{j=1}^{b} \rho_{j}\right)>0, \quad \nu(\bar{n}) \leqslant \operatorname{Im}\left(\sum_{j=1}^{b} \rho_{j}\right)<\nu(\bar{n})+b .
$$

Поэтому

$$
\left|1+\sum_{j=1}^{b} \rho_{j}\right|^{-1} \leqslant a(\nu)= \begin{cases}1, & |\nu| \leqslant b \\ (|\nu|-b)^{-1}, & |\nu|>b .\end{cases}
$$

Количество нулей $\zeta(s)$ с учетом кратности в прямоугольнике $\{s \in \mathbb{C} \mid 0<\operatorname{Re} s<1$, $T \leqslant \operatorname{Im} s<T+1\}$ есть $O(\ln |T|)$, к тому же [27] мнимые части нетривиальных нулей $\zeta$ по абсолютной величине больше 14. Ввиду сказанного получаем оценку

$$
A(\bar{n}) \leqslant \begin{cases}a(\nu)\left(c_{31}\right)^{b} \prod_{j=1}^{b}\left(\frac{\ln \left|n_{j}\right|}{\left|n_{j}\right|-1}\right), & \min \left|n_{j}\right| \geqslant 14 \\ 0, & \min \left|n_{j}\right|<14 .\end{cases}
$$


Отсюда находим

$$
M_{1}(b) \leqslant\left(c_{31}\right)^{b} M_{2}(b)
$$

где

$$
\begin{gathered}
M_{2}(b)=\sum_{\nu \in \mathbb{Z}} a(\nu) \sigma(\nu), \\
\sigma(\nu)=\sum_{\substack{n_{1} \in \mathbb{Z} \\
n_{1}+\cdots+\sum_{n_{b} \in \mathbb{Z}} \\
\min \left|n_{j}\right| \geqslant 14}} \prod_{j=1}^{b}\left(\frac{\ln \left|n_{j}\right|}{\left|n_{j}\right|-1}\right) .
\end{gathered}
$$

Воспользовавшись равенством Парсеваля, получаем следуюшее представление для суммы $M_{2}(b)$ :

$$
M_{2}(b)=\int_{0}^{1} f_{1}(t) f_{2}(t) d t
$$

где

$$
\begin{gathered}
f_{1}(t)=\sum_{\nu \in \mathbb{Z}} a(\nu) \exp \{-2 \pi i \nu t\} \\
f_{2}(t)=\left(f_{3}(t)\right)^{b}, \quad f_{3}(t)=\sum_{\substack{\nu \in \mathbb{Z} \\
|n| \geqslant 14}} \frac{\ln |n|}{|n|-1} \exp \{2 \pi i n t\}
\end{gathered}
$$

Учитывая четность коэффициентов Фурье функций $f_{1}$ и $f_{3}$, приходим к соотношениям

$$
M_{2}(b)=2^{b+2} \int_{0}^{1 / 2} f_{4}(t)\left(f_{5}(t)\right)^{b} d t
$$

где

$$
\begin{aligned}
& f_{4}(t)=\frac{1}{2}+\sum_{\nu=1}^{\infty} a(\nu) \cos (2 \pi \nu t), \\
& f_{5}(t)=\sum_{n=14}^{\infty} \frac{\ln n}{n-1} \cos (2 \pi n t) .
\end{aligned}
$$

Стандартным образом [28, гл. 10, с. 671] получаем оценки функций (7.3):

$$
\begin{aligned}
& \left|f_{4}(t)\right| \leqslant c_{32} b|\ln t|, \quad 0<t<1 / 2 . \\
& \left|f_{5}(t)\right| \leqslant c_{33} \ln ^{2} t,
\end{aligned}
$$

Из (8.3) и (6.3) находим

$$
M_{2}(b) \leqslant\left(c_{34} b\right)^{2 b},
$$

откуда, учитьвая (5.3), получаем (4.3). Как отмечалось вьше, это доказывает теоремy 1. 
ДОКАЗАТЕЛЬСТво СЛЕДСТВИЯ 1. Обозначим

$$
\Phi_{b}(u)=\int_{1}^{u}|R(t)|^{b} d t
$$

Интегрируя по частям, с учетом (1.2) находим

$$
\begin{aligned}
\int_{1}^{x}|\Delta(u)|^{b} d u & =\int_{1}^{x}|R(u)|^{b} u^{-b \Theta} d u \\
& =\Phi_{b}(x) x^{-b \Theta}+b \Theta \int_{1}^{x} \Phi_{b}(u) u^{-1-b \Theta} d u \\
& \leqslant x\left(c_{7} b\right)^{2 b}+b \Theta \int_{1}^{x}\left(c_{7} b\right)^{2 b} d u<x\left(c_{7} b\right)^{2 b}(1+b) .
\end{aligned}
$$

Следствие 1 доказано.

Следствие 6 выводится из (9.3) аналогичным приемом.

ДокАЗАТЕЛЬСТво СЛЕДСТВИЯ 2. Имеем

$$
\exp \{\lambda \sqrt{|\Delta(u)|}\}=\sum_{n=0}^{\infty} \frac{\lambda^{n}|\Delta(u)|^{n / 2}}{n !}
$$

Согласно (9.3)

$$
x^{-1} \int_{1}^{x}|\Delta(u)|^{n / 2} d u \leqslant(1+n / 2)\left(c_{7} n / 2\right)^{n}, \quad n \geqslant 2,
$$

и очевидно, что

$$
x^{-1} \int_{1}^{x}\left(|\Delta(u)|^{0}+\lambda|\Delta(u)|^{1 / 2}\right) d u \leqslant c_{35}
$$

Из (10.3)-(12.3) находим

$$
\begin{aligned}
x^{-1} \int_{1}^{x} \exp \{\lambda \sqrt{|\Delta(u)|}\} d u & =\sum_{n=0}^{\infty} \frac{\lambda^{n}}{n !} \cdot \frac{1}{x} \int_{1}^{x}|\Delta(u)|^{n / 2} d u \\
& \leqslant c_{35}+\sum_{n=2}^{\infty}\left(1+\frac{n}{2}\right) \frac{\left(\lambda c_{7} n / 2\right)^{n}}{n !} .
\end{aligned}
$$

Ясно, что если $\lambda \leqslant\left(e c_{7}\right)^{-1}$, то сумма ряда в $(13.3)$ не превосходит некоторой абсолютной постоянной. Тем самым следствие 2 доказано. 
ДоКАЗАТЕЛЬСтво СЛЕДСтвия 3. Согласно следствию 2 для $c_{36}=\left(e c_{7}\right)^{-1}$ имеем

$$
I=\int_{1}^{2 x} \exp \left\{c_{36} \sqrt{|\Delta(u)|}\right\} d u=O(x)
$$

С другой стороны, выполнено очевидное неравенство

$$
I \geqslant I_{1}=\int_{E} \exp \left\{c_{36} \sqrt{|\Delta(u)|}\right\}
$$

где

$$
E=\{u \in[x, 2 x]|| \Delta(u) \mid \geqslant V(u)\}
$$

Из (15.3) и (16.3) ввиду монотонности функции $V$ получаем

$$
I_{1} \geqslant \mathfrak{M}(V, x) \exp \left\{c_{36} \sqrt{V(x)}\right\}
$$

Из (14.3), (15.3) и (17.3) вытекает (4.2). Следствие 3 доказано.

Для дальнейшего нам потребуется

ЛЕмма 1. Пусть

$$
Q(x)=\int_{2}^{x} \frac{|R(t)| d t}{t \ln ^{2} t} .
$$

Тогда при любых $x \geqslant 2$ с некоторой абсолютной постоянной $c_{37}$ выполняется неравенство

$$
Q(x) \leqslant c_{37} x^{\Theta} \ln ^{-2} x .
$$

ДокАЗАТЕльСтво. Сохраняя обозначение, введенное в доказательстве следствия 1 , имеем

$$
Q(x)=\left.\frac{\Phi_{1}(t)}{t^{2} \ln ^{2} t}\right|_{2} ^{x}+\int_{2}^{x} \Phi_{1}(t)\left(\frac{1}{t^{2} \ln ^{2} t}+\frac{2}{t^{2} \ln ^{3} t}\right) d t .
$$

Из (2.2) и (19.3) находим

$$
Q(x) \leqslant c_{8} x^{\Theta} \ln ^{-2} x+c_{8} \int_{2}^{x} t^{-\Theta-1}\left(\ln ^{-2} t+2 \ln ^{-3} t\right) d t \leqslant c_{37} x^{\Theta} \ln ^{-2} x .
$$

Лемма 1 доказана. 
ДОКАЗАТЕЛЬСТВО СЛЕДСТВИЯ 4. Из (1.1) и (18.3) находим

$$
|P(x)| \leqslant \frac{|R(x)|}{\ln x}+c_{38} \frac{x^{\Theta}}{\ln x} \quad(\forall x \geqslant 2) .
$$

Так как при любых $b \geqslant 1, \alpha, \beta \geqslant 0$ справедливо неравенство $(\alpha+\beta)^{b} \leqslant 2^{b-1}\left(\alpha^{b}+\beta^{b}\right)$, то

(20.3)

$$
\begin{aligned}
\int_{2}^{x}|P(u)|^{b} d u & \leqslant 2^{b-1}\left(\int_{2}^{x}|R(u)|^{b}(\ln u)^{-b} d u+c_{38}^{b} \int_{2}^{x} u^{b \Theta}(\ln u)^{-b} d u\right) \\
& =2^{b-1}\left(\left.\frac{\Phi_{b}(u)}{(\ln u)^{b}}\right|_{2} ^{x}+b \int_{2}^{x} \frac{\Phi(u) d u}{u(\ln u)^{b+1}}+c_{38}^{b} \int_{2}^{x} \frac{u^{b \Theta}}{(\ln u)^{b}} d u\right) \\
& <\left(c_{39} b\right)^{2 b}\left(x^{1+b \Theta}(\ln x)^{-b}+\int_{2}^{x} u^{b \Theta}(\ln u)^{-b} d u\right) .
\end{aligned}
$$

Проверим, что при любых $b \geqslant 1, \alpha \geqslant b / 2$ и $x \geqslant 4$ имеет место оценка

$$
I(x, b, \alpha)=\int_{2}^{x} u^{\alpha}(\ln u)^{-b} d u \leqslant 6^{b} x^{\alpha+1}(\ln x)^{-b} .
$$

Действительно,

$$
\begin{aligned}
I(x, b, \alpha) & =\int_{2}^{\sqrt{x}} u^{\alpha}(\ln u)^{-b} d u+\int_{\sqrt{x}}^{x} u^{\alpha}(\ln u)^{-b} d u \\
& \leqslant(\ln 2)^{-b} x^{1+\alpha / 2}+\left(\frac{1}{2} \ln x\right)^{-b}+\int_{\sqrt{x}}^{x} u^{\alpha} d u \\
& \leqslant 2^{b} x^{\alpha+1}\left(x^{-\alpha / 2}+(\ln x)^{-b}\right) \\
& \leqslant 2^{b} x^{\alpha+1}(\ln x)^{-b}\left(1+\left(x^{-1 / 4} \ln x\right)^{b}\right) .
\end{aligned}
$$

(В последнем переходе мы использовали, что $\alpha \geqslant b / 2$ и, следовательно, $x^{-\alpha / 2} \leqslant$ $x^{-b / 4}$.) Поскольку $\max _{x \geqslant 4}\left(x^{-1 / 4} \ln x\right)=4 / e$, окончательно получаем

$$
I(x, b, \alpha) \leqslant 2^{b} x^{\alpha+1}(\ln x)^{-b}\left(1+(4 / e)^{b}\right)<6^{b} x^{\alpha+1}(\ln x)^{-b} .
$$

Неравенство (21.3) доказано. Из (21.3) при $\alpha=b \Theta$ получаем (20.3), а из (20.3), в свою очередь, вытекает (5.2).

Перед тем, как приступить к доказательствам теорем 2 и 3 , введем несколько обозначений и установим справедливость одной леммы. Положим

$$
\begin{aligned}
& \mathscr{F}_{1}(x, \sigma)=\sum_{\operatorname{Re} \rho<\sigma} x^{\rho} / \rho, \\
& \mathscr{F}_{2}(x, \sigma)=\mathscr{F}(x)-\mathscr{F}_{1}(x, \sigma)=\sum_{\operatorname{Re} \rho \geqslant \sigma} x^{\rho} / \rho .
\end{aligned}
$$

Из простейшей плотностной теоремы о нулях дзета-функции Римана [21, с. 237], которая утверждает, что количество нулей $\zeta(s)$ в прямоугольнике $\{s \in \mathbb{C} \mid \sigma \leqslant \operatorname{Re} s<1$, $|\operatorname{Im} s| \leqslant T\}$ есть $O\left(T^{3 / 2-\sigma} \ln ^{5} T\right)$, вытекает, что при $\sigma>1 / 2$

$$
S(\sigma)=\sum_{\operatorname{Re} \rho \geqslant \sigma}|1 / \rho|<+\infty .
$$

Следовательно, ряд (23.3) абсолютно сходится при всех $x>0$, а ряд (22.3) сходится в каждой точке $x \in(1,+\infty)$, как и ряд $(37.2)$ для $\mathscr{F}(x)$. 
ЛЕмма 2. Если $\sigma>1 / 2$, mo

$$
\mathscr{F}_{1}(x, \sigma)=o\left(x^{\sigma}\right) \quad(x \rightarrow+\infty) .
$$

ДокАЗАТЕЛЬСТво. Обозначим $B=(\sigma-1 / 2)^{-1}$,

$$
U(x)=\sum_{|\operatorname{Im} \rho| \leqslant x^{B}} x^{\rho} / \rho, \quad U_{1}(x, \sigma)=\sum_{\substack{|\operatorname{Im} \rho| \leqslant x^{B} \\ \operatorname{Re} \rho<\sigma}} x^{\rho} / \rho .
$$

Известно [3, с. 122 , формулы $(9)-(10)]$ что $^{2}$

$$
\mathscr{F}(x)=U(x)+O(\ln x) .
$$

Отсюда находим

$$
\begin{aligned}
\mathscr{F}_{1}(x, \sigma) & =\mathscr{F}(x)-\mathscr{F}_{2}(x, \sigma)=U(x)-\mathscr{F}_{2}(x, \sigma)+O(\ln x) \\
& =U_{1}(x, \sigma)-\sum_{\substack{\left|\operatorname{Im}_{\operatorname{li}} \rho\right|>x^{B} \\
\operatorname{Re} \rho \geqslant \sigma}} x^{\rho} / \rho+O(\ln x) .
\end{aligned}
$$

Из упомянутой выше плотностной теоремы вытекает, что при любых $\sigma>1 / 2$ и $d>2$

$$
\sum_{\substack{\operatorname{Re} \rho \geqslant \sigma \\|\operatorname{Im} \rho|>d}}|1 / \rho|=O_{\sigma}\left(d^{1 / 2-\sigma} \ln ^{5} d\right)
$$

Это вместе с (26.3) дает

$$
\begin{aligned}
\mathscr{F}_{1}(x, \sigma) & =U_{1}(x, \sigma)+O_{\sigma}\left(x^{\Theta+B(1 / 2-\sigma)} \ln ^{5} x+\ln x\right) \\
& =U_{1}(x, \sigma)+O_{\sigma}\left(\ln ^{5} x\right) .
\end{aligned}
$$

Осталось показать, что $U_{1}(x, \sigma)=o\left(x^{\sigma}\right)(x \rightarrow+\infty)$. Возьмем произвольное $\varepsilon>0$ и зафиксируем его. Положим $\sigma_{1}=1 / 4+\sigma / 2$. Ясно, что $1 / 2<\sigma_{1}<\sigma$. В силу (24.3) найдется $T=T(\varepsilon)$ такое, что

$$
\sum_{\substack{\operatorname{Re} \rho \geqslant \sigma_{1} \\|\operatorname{Im} \rho|>T}}|1 / \rho|<\varepsilon / 3
$$

Теперь воспользуемся очевидным представлением

$$
U_{1}(x, \sigma)=U_{2}(x)+U_{3}(x)+U_{4}(x),
$$

где

$$
U_{2}(x)=\sum_{\substack{\operatorname{Re} \rho<\sigma_{1} \\|\operatorname{Im} \rho| \leqslant x^{B}}} x^{\rho} / \rho, \quad U_{3}(x)=\sum_{\substack{\sigma_{1} \leqslant \operatorname{Re} \rho<\sigma \\ T<|\operatorname{Im} \rho| \leqslant x^{B}}} x^{\rho} / \rho, \quad U_{4}(x)=\sum_{\substack{\sigma_{1} \leqslant \operatorname{Re} \rho<\sigma \\|\operatorname{Im} \rho| \leqslant T}} x^{\rho} / \rho .
$$

\footnotetext{
${ }^{2}$ В [3] дана существенно лучшая, чем (25.3), оценка, но она нам не потребуется.
} 
Оценим каждую из сумм в отдельности. Поскольку (см. [3, с. 124-125])

$$
\sum_{|\operatorname{Im} \rho| \leqslant y}|1 / \rho|=O\left(\ln ^{2} y\right),
$$

то

$$
\left|U_{2}(x)\right| \leqslant x^{\sigma_{1}} \sum_{|\operatorname{Im} \rho| \leqslant x^{B}}|1 / \rho| \leqslant c_{40} B^{2} x^{\sigma_{1}} \ln ^{2} x .
$$

Ввиду (28.3)

$$
U_{3}(x) \leqslant x^{\sigma} \sum_{\substack{\operatorname{Re} \rho \geqslant \sigma_{1} \\|\operatorname{Im} \rho|>T}}|1 / \rho|<x^{\sigma} \varepsilon / 3 .
$$

Наконец,

$$
\left|U_{4}(x)\right| \leqslant x^{\alpha} \sum_{|\operatorname{Im} \rho| \leqslant T}|1 / \rho| \leqslant c_{40} x^{\alpha} \ln T,
$$

где

$$
\alpha=\max \{\operatorname{Re} \rho|\operatorname{Re} \rho<\sigma,| \operatorname{Im} \rho \mid \leqslant T\} .
$$

Поскольку $\alpha$ вместе с $T$ зависит только от $\varepsilon$ и $\alpha<\sigma$, то найдется такое число $T_{1}=T_{1}(\varepsilon)$, что при любых $x>T_{1}$ будут выполняться неравенства

$$
\begin{array}{r}
c_{40} B^{2} x^{\sigma_{1}-\sigma} \ln ^{2} x<\varepsilon / 3 \\
c_{40} x^{\alpha-\sigma} \ln ^{2} T<\varepsilon / 3 .
\end{array}
$$

Тогда окажется, что при $\forall x>T_{1}$

$$
\left|U_{j}(x)\right|<x^{\sigma} \varepsilon / 3, \quad j=2,3,4 .
$$

Складывая оценки (30.3) для сумм $U_{j}$, приходим ввиду (29.3) к следуюшему утверждению. Для любого $\varepsilon>0$ сушествует $T_{1}=T_{1}(\varepsilon)$ такое, что при всех $x>T_{1}$ справедливо неравенство $\left|U_{1}(x)\right|<\varepsilon x^{\sigma}$. Это и означает, что $U_{1}(x)=o\left(x^{\sigma}\right)(x \rightarrow+\infty)$. Последнее соотношение вместе с (27.3) убеждает нас в справедливости леммы 2.

ДОКАЗАТЕЛЬСТво тЕОРЕмЫ 2. Если $\Theta$ недостижимо, то теорема 2 сразу же получается из леммы 2 при $\sigma=\Theta$. Если $\Theta>1 / 2$ достижимо, то согласно лемме 2

$$
\mathscr{F}(x)=\mathscr{F}_{2}(x, \Theta)+o\left(x^{\Theta}\right) \quad(x \rightarrow+\infty) .
$$

Ввиду (24.3) и определений $\mathscr{F}_{2}(x, \Theta)$ и $\Theta$ имеем

$$
\left|\mathscr{F}_{2}(x, \Theta)\right| \leqslant x^{\Theta} S(\Theta)
$$

Из (31.3) и (32.3) получаем (3.2). Теорема 2 доказана.

Следствие 5 непосредственно вытекает из теоремы 2, леммы 1 и соотношения (1). 
ДокАЗАТЕЛЬСТВо тЕорЕмы 3. Если $\Theta$ недостижимо, то по теореме 2 имеем $\Delta(u)=o(1)(u \rightarrow+\infty)$, и, следовательно,

$$
\lim _{x \rightarrow+\infty}\left((\ln x)^{-1} \int_{1}^{x}(\Delta(u))^{b} u^{-1} d u\right)=0 .
$$

С другой стороны, сумма в (7.2) пуста, и, значит, $B=0$. Таким образом, равенство (6.2) в этом случае доказано. Если $\Theta$ достижимо, то согласно лемме 2

$$
\Delta(u)=\mathscr{F}_{2}(u) u^{-\Theta}+o(1) \quad(u \rightarrow+\infty),
$$

и, следовательно, достаточно доказать, что

$$
B(b)=\lim _{x \rightarrow+\infty}\left((\ln x)^{-1} \int_{1}^{x}\left(\mathscr{F}_{2}(u), \Theta\right) u^{-1} d u\right)
$$

Сделав замену переменного $u=e^{t}$ и обозначив $y=\ln x, \gamma=\operatorname{Im} \rho$, получим соотношение, равносильное (33.3):

$$
B(b)=\lim _{y \rightarrow+\infty}\left(y^{-1} \int_{0}^{y}\left(\sum_{\operatorname{Re} \rho=\Theta} e^{i \gamma t} / \rho\right)^{b} d t\right) .
$$

Если $\Theta>1 / 2$, то соотношение (34.3) является почти очевидным. Поскольку

$$
\sum_{\operatorname{Re} \rho=\Theta>1 / 2}|1 / \rho|<+\infty,
$$

функция

$$
\varphi(t)=\sum_{\operatorname{Re} \rho=\Theta} e^{i \gamma t} / \rho
$$

является почти периодической функцией Бора [29] с абсолютно сходящимся рядом экспонент. Ясно, что и при любом $b \in \mathbb{N}$ функция $(\varphi(t))^{b}$ обладает тем же свойством. Согласно теореме из [30] предел, стояший в правой части выражения (34.3), равен свободному члену в разложении $(\varphi(t))^{b}$ в ряд экспонент. С другой стороны, возводя в степень ряд (35.3), убеждаемся в том, что свободный член получившегося ряда равен сумме $B(b)$.

Если $\Theta=1 / 2$, то $\varphi(t)$ является почти периодической функцией Безиковича класса $B_{2} \subset B_{1}$, но не Бора. ${ }^{3}$ Упомянутая вьше теорема из [30] доказывается для функций из $B_{1}$. Поэтому нам остается только установить, что в случае справедливости гипотезы Римана любая целая степень $\varphi(t)$ лежит в $B_{1}$, а ряд экспонент, сходящийся к ней по метрике этого пространства, получается после возведения в ту же степень ряда (35.3). Последнее означает, что

$$
\lim _{N \rightarrow \infty}\left(\limsup _{y \rightarrow+\infty} J(N, y)\right)=0
$$

\footnotetext{
${ }^{3}$ Определения этих пространств функций и стандартных метрик в них см. в [29].
} 
где

$$
J(N, y)=\frac{1}{y} \int_{-y}^{y}\left|(\varphi(N, t))^{b}-(\varphi(t))^{b}\right| d t, \quad \varphi(N, t)=\sum_{|\gamma| \leqslant N} e^{i \gamma t} / \rho .
$$

При любых $x_{1}, x_{2} \in \mathbb{R}$ и $b \in \mathbb{N}$ имеем

$$
\begin{aligned}
\left|x_{1}^{b}-x_{2}^{b}\right| & =\left|x_{1}-x_{2}\right| \cdot\left|\sum_{k=0}^{b} x_{1}^{k} x_{2}^{b-k}\right| \\
& \leqslant\left|x_{1}-x_{2}\right| \cdot \sum_{k=0}^{b}\left|x_{1}\right|^{k}\left|x_{2}\right|^{b-k} \\
& \leqslant\left|x_{1}-x_{2}\right| \cdot \frac{1}{2} \sum_{k=0}^{b}\left(\left|x_{1}\right|^{2 k}+\left|x_{2}\right|^{2 b-2 k}\right) \\
& =\frac{1}{2} \sum_{k=0}^{b-1}\left(\left|x_{1}-x_{2}\right|\left|x_{1}\right|^{2 k}+\left|x_{1}-x_{2}\right|\left|x_{2}\right|^{2 k}\right) .
\end{aligned}
$$

Используя (37.3) для $x_{1}=\varphi(N, t), x_{2}=\varphi(t)$ и далее неравенство Коши-Буняковского для интегралов, получаем

$$
\begin{aligned}
J(N, y)< & \left(\frac{1}{y} \int_{-y}^{y}(\varphi(N, t)-\varphi(t))^{2} d t\right)^{1 / 2} \\
& \times \sum_{k=0}^{b-1}\left(\left(\frac{1}{y} \int_{-y}^{y}(\varphi(N, t))^{4 k} d t\right)^{1 / 2}+\left(\frac{1}{y} \int_{-y}^{y}(\varphi(t))^{4 k} d t\right)^{1 / 2}\right) .
\end{aligned}
$$

Вспомним теперь результат следствия 6 и его доказательство. Если положить $y=\ln x, u=e^{t}$, то следствие 6 при $\Theta=1 / 2$ утверждает, что

$$
\int_{0}^{y}\left|R\left(e^{t}\right) e^{-t / 2}\right|^{q} d t \leqslant c_{41}(q) y \quad(\forall q \geqslant 1, \forall y \geqslant 1) .
$$

Соотношение (39.3) выводилось из основного неравенства (1.2) теоремы 1. А в процессе доказательства теоремы 1 было видно, что оценка, стоящая в правой части (1.2), годится и для интегралов от степеней модуля всех сумм $\sum_{|\gamma| \leqslant N} x^{\rho} / \rho$. Всюду мы опираемся на то, что $R$ "мало отличается" от $\mathscr{F}($ см. (31.3)). А так как в случае $\Theta=1 / 2$ имеем $\mathscr{F}\left(e^{t}\right) e^{-t / 2}=\varphi(t)$, то в действительности было показано, что при любом $q \geqslant 1$

$$
\begin{gathered}
\sup _{y \geqslant 1} \frac{1}{y} \int_{0}^{y}|\varphi(t)|^{q} d t \leqslant c_{41}(q), \\
\sup _{y \geqslant 1} \sup _{N>0} \frac{1}{y} \int_{0}^{y}|\varphi(N, t)|^{q} d t \leqslant c_{41}(q) .
\end{gathered}
$$

Нетрудно проверить справедливость тождества

$$
\varphi(t)+\varphi(-t)=\sum_{\gamma} \frac{\cos \gamma t}{\gamma^{2}+1 / 4} \quad(\Theta=1 / 2) .
$$


Это означает, что $\varphi(-t)$ и $\varphi(N,-t)$ отличаются соответственно от $\varphi(t)$ и $\varphi(N, t)$ на $O(1)$ равномерно по $N$ и $t$. Поэтому оценки (40.3) переносятся на интегралы по отрезкам $[-y, y]$ :

$$
\begin{gathered}
\sup _{y \geqslant 1} \frac{1}{y} \int_{-y}^{y}|\varphi(t)|^{q} d t \leqslant c_{42}(q), \\
\sup _{y \geqslant 1} \sup _{N>0} \frac{1}{y} \int_{-y}^{y}|\varphi(N, t)|^{q} d t \leqslant c_{42}(q) .
\end{gathered}
$$

Эти неравенства вместе с (38.3) приводят к оценке

$$
J(N, y)<c_{43}(q)\left(\frac{1}{y} \int_{-y}^{y}(\varphi(N, t)-\varphi(t))^{2} d t\right)^{1 / 2}
$$

Ввиду сходимости ряда $\sum_{\rho}|1 / \rho|^{2}$ справедливо включение $\varphi \in B_{2}$. Поэтому ее ряд экспонент (35.3) сходится к $\varphi$ по метрике этого пространства. То есть

$$
\lim _{N \rightarrow \infty}\left(\limsup _{y \rightarrow+\infty} \frac{1}{2 y} \int_{-y}^{y}(\varphi(N, t)-\varphi(t))^{2} d t\right)^{1 / 2}=0 .
$$

Из (42.3) и (41.3) получаем (36.3). Как отмечалось выше, это доказывает теорему 3 и в случае $\Theta=1 / 2$.

Заметим, что обобщенная нами теорема Крамера, представляющая частный случай теоремы $3(b=2$ и $\Theta=1 / 2)$ с точки зрения теории почти периодических функций, является тривиальным замечанием. Это - формула среднего для $\varphi^{2}$. Ясно, что она верна, так как $\varphi^{2} \in B_{1}$, поскольку $\varphi \in B_{2}$.

\section{§4. Доказательства теорем 4-14 и их следствий}

Докажем сначала теоремы 6-11. Из теорем 6, 7 очевидным образом будет следовать теорема 4, а из теорем 9-11 - теорема 5. Доказательству теоремы 6 предпошлем две леммы и введем еше несколько обозначений. Положим

$$
\begin{gathered}
\alpha=\frac{\ln 2}{8}, \quad g(z)=\left(\frac{\sin \alpha z}{\alpha z}\right)^{4}\left(1-\frac{z}{12}\right), \\
\widehat{g}(y)=\frac{1}{2 \pi} \int_{-\infty}^{+\infty} g(z) \exp (-i z y) d z .
\end{gathered}
$$

Ясно, что $g \in L^{2}(\mathbb{R})$ и является целой функцией экспоненциального типа $4 \alpha$. Поэтому

$$
\begin{gathered}
\operatorname{supp} \widehat{g} \subset[-4 \alpha, 4 \alpha] \\
g(z)=\int_{-\infty}^{+\infty} \widehat{g}(y) \exp (i y z) d y=\int_{-4 \alpha}^{4 \alpha} \widehat{g}(y) \exp (i y z) d y .
\end{gathered}
$$

ЛЕмма 3.

$$
\max _{y \in \mathbb{R}}|\widehat{g}(y)|=\frac{8}{3 \ln 2}
$$


ДоКАЗАТЕЛЬСТВО. Положим

$$
G(y)= \begin{cases}0, & |y| \geqslant 4, \\ \frac{|y|-4}{16}, & 2 \leqslant|y|<4, \\ \frac{4-3|y|}{16}, & 0 \leqslant|y|<2 .\end{cases}
$$

Нетрудно убедиться в справедливости тождества

$$
\int_{\infty}^{+\infty} G(y) \exp (i y z) d y=\frac{\sin ^{4} z}{z^{2}} \quad \forall z \in \mathbb{R}
$$

Следовательно,

$$
G(y)=\frac{1}{2 \pi} \int_{-\infty}^{+\infty} \frac{\sin ^{4} z}{z^{2}} \exp (-i y z) d z
$$

Пусть $G_{1}(y)=\int_{0}^{y} G(t) d t$, т.е. $G_{1}-$ первообразная для $G$, которая обрашается в нуль в точке $y=0$. Интегрируя по $y$ под знаком интеграла (4.4), находим

$$
G_{1}(y)=-\frac{1}{2 \pi i} \int_{-\infty}^{+\infty} \frac{\sin ^{4} z}{z^{3}} \exp (-i y z) d z
$$

С другой стороны, интегрируя (3.4), получаем явную формулу для $G_{1}(y)$ :

$$
G_{1}(y)= \begin{cases}\frac{8 y-3 y^{2}}{32}, & 0 \leqslant y \leqslant 2 \\ \frac{(y-4)^{2}}{32}, & 2<y<4 \\ 0, & y \geqslant 4 \\ -G_{1}(-y), & y<0\end{cases}
$$

Пусть

$$
G_{2}(y)=\frac{1}{2 \pi} \int_{-\infty}^{+\infty}\left(\frac{\sin z}{z}\right)^{4} \exp (-i y z) d z
$$

Так как $G_{2}-$ преобразование Фурье целой функции $\left(\frac{\sin z}{z}\right)^{4}$ экспоненциального типа 4 из класса $L_{2}(\mathbb{R})$, то $\operatorname{supp} G_{2} \subset[-4,4]$. Имеем также

$$
G_{2}^{\prime}(y)=-G_{1}(y), \quad G_{2}(0)=1 / 3 .
$$

Легко видеть, что функция $\widehat{g}$, определенная в (1.4), выражается через $G_{1}$ и $G_{2}$ следуюшим образом:

$$
\widehat{g}(y)=\frac{1}{\alpha}\left(G_{2}\left(\frac{y}{\alpha}\right)+\frac{i}{12 \alpha} G_{1}\left(\frac{y}{\alpha}\right)\right) .
$$


Отсюда находим

$$
|\widehat{g}(y)|=\frac{1}{\alpha} \sqrt{G_{2}^{2}\left(\frac{y}{\alpha}\right)+\left(\frac{2}{3 \ln 2}\right)^{2} G_{1}^{2}\left(\frac{y}{\alpha}\right)} .
$$

Поскольку $\widehat{g}$ - непрерьвная на $\mathbb{R}$ функция, обращающаяся в нуль вне $(-4 \alpha, 4 \alpha)$, ясно, что максимум $|\widehat{g}(y)|$ на $\mathbb{R}$ сушествует и лежит на интервале $(-4 \alpha, 4 \alpha)$. Но, как мы сейчас установим, функция $G_{2}^{2}(y / \alpha)+k^{2} G_{1}^{2}(y / \alpha), k=2 /(3 \ln 2)$, имеет на интервале $(-4 \alpha, 4 \alpha)$ единственньй локальный экстремум в точке $y=0$. Отсюда и будет следовать, что

$$
\max _{y \in \mathbb{R}}|\widehat{g}(y)|=|\widehat{g}(0)|=\frac{1}{\alpha}\left|G_{2}(0)\right|=\frac{8}{3 \ln 2} .
$$

Итак, проверим, что функция $H(x)=G_{2}^{2}(x)+\lambda G_{1}^{2}(x)$ при $0<\lambda<1$ имеет на интервале $(-4,4)$ единственньй локальньй экстремум в точке $x=0$. Имеем:

$$
\begin{aligned}
H^{\prime}(x) & =2 G_{2}(x) G_{2}^{\prime}(x)+2 \lambda G_{1}(x) G_{1}^{\prime}(x) \\
& =-2 G_{2}(x) G_{1}(x)+2 \lambda G_{1}(x) G(x) \\
& =-2 G_{1}(x)\left(G_{2}(x)-\lambda G(x)\right)
\end{aligned}
$$

Из $(5.4)$ следует, что $G_{1}(x)$ имеет на $(-4,4)$ единственньй нуль в точке $x=0$. Поэтому осталось показать, что

$$
H_{1}(x)=G_{2}(x)-\lambda G(x) \neq 0 \quad \forall x \in(-4,4) .
$$

При $x \in(4 / 3,4)$ неравенство (6.4) очевидно, так как на этом интервале $G(x)<0$ (см. $(3.4))$, а $G_{2}(x)>0$. (Если бы на интервале $(0,4)$ нашлась точка $x_{0}$, в которой $G_{2}\left(x_{0}\right) \leqslant 0$, то ввиду того, что $G_{2}(0)>0$, нашлась бы и точка $x_{1} \in(0,4)$, в которой $G_{2}\left(x_{1}\right)=0$. Но тогда по теореме Ролля на интервале $\left(x_{1}, 4\right)$ функция $G_{2}^{\prime}(x)=-G_{1}(x)$ имела бы нуль, а это, как отмечалось вьше, неверно.) На $[0,4 / 3]$ проверить (6.4) тоже несложно. При этих значениях $x$ имеем

$$
\begin{aligned}
H_{1}(x) & \geqslant G_{2}(x)-G(x)=H_{2}(x)=H_{2}(0)+\int_{0}^{x} H_{2}^{\prime}(t) d t \\
& =\frac{1}{3}-\frac{1}{4}+\int_{0}^{x}\left(G_{2}^{\prime}(t)-G^{\prime}(t)\right) d t=\frac{1}{12}+\int_{0}^{x}\left(\frac{3}{16}-G_{1}(t)\right) d t .
\end{aligned}
$$

Из (5.4) видно, что $G_{1}(t) \leqslant 3 / 16 \forall t \in(0,4 / 3)$. Поэтому $H_{1}(x) \geqslant H_{2}(x)>0$. Таким образом, мы установили (6.4) при $\forall x \in[0,4)$, а значит, и при любых $x \in(-4,4)$ ввиду четности $H_{1}$. Тем самым лемма 3 полностью доказана.

Лемма 4. Справедливы следующие неравенства:

$$
\begin{aligned}
& |g(x)|<\frac{0.8}{x+14.5} \quad \forall x \geqslant 26, \\
& |g(x)|<\frac{0.8}{|x+14|} \quad \forall x \leqslant-40 .
\end{aligned}
$$


ДоказАТЕльство. Неравенство (7.4) установим по отдельности на промежутках $\delta_{1}=[26,46]$ и $\delta_{2}=(46,+\infty)$. Из определения функции $g($ см. (1.4)) получаем

$$
|g(x)|=\frac{a(x)(\sin \alpha x)^{4}}{12 \alpha^{4}(x+14.5)}
$$

где $a(x)=x^{-4}(x+14.5)(x-12)$. Нетрудно убедиться в том, что при $x \geqslant 20$ функция $a(x)$ положительна и строго убывает. Ввиду сказанного приходим к оценкам

$$
\begin{aligned}
& \sup _{x \in \delta_{2}}|g(x)(x+14.5)| \leqslant \frac{a(46)}{12 \alpha^{4}}, \\
& \max _{x \in \delta_{1}}|g(x)(x+14,5)| \leqslant \frac{a(26)}{12 \alpha^{4}} \cdot \max _{x \in \delta_{1}}\left(\sin ^{4} \alpha x\right) .
\end{aligned}
$$

Легко проверить, что $\max _{x \in \delta_{1}}\left(\sin ^{4} \alpha x\right)<0.4$, и после этого непосредственным подсчетом установить, что правые части неравенств (9.4) и (10.4) меньше 0.8. Тем самьг (7.4) доказано. Аналогичным образом доказывается и неравенство (8.4). Имеем:

$$
|g(x)|=\frac{a_{1}(x) \sin ^{4} \alpha x}{|x+14| \cdot 12 \alpha^{4}} \leqslant \frac{a_{1}(x)}{|x+14| \cdot 12 \alpha^{4}}
$$

где $a_{1}(x)=|x+14||x-12| x^{4}$. При $x \leqslant-20$ функция $a_{1}(x)$ строго возрастает, следовательно,

$$
\max _{x \leqslant-40}(|x+14||g(x)|) \leqslant \frac{a_{1}(-40)}{12 \alpha^{4}}<0.8
$$

а это и означает справедливость (8.4). Лемма 4 полностью доказана.

Напомним, что в теореме 6 рассматривается случай $\Theta=1 / 2$. Это означает, что все нетривиальные нули $\zeta(s)$ лежат на прямой $\operatorname{Re} s=1 / 2$. Нам будет удобно расположить их в последовательность $\left\{\rho_{n}=1 / 2+i \gamma_{n}\right\}_{n \in \mathbb{Z}, n \neq 0}$ в порядке возрастания их мнимых частей. Если нуль оказался кратньм, то он записьвается в получившуюся последовательность столько раз, какова его кратность. Наконец, $\gamma_{-n}=-\gamma_{n} \forall n \in \mathbb{N}$. Положим

$$
\varphi(t)=\sum_{\substack{n=-\infty \\ n \neq 0}}^{+\infty} \rho_{n}^{-1} \exp \left(i \gamma_{n} t\right)
$$

Из (27.2) следует, что

$$
\mathscr{F}\left(e^{t}\right) e^{-t / 2}=\varphi(t) \quad(\Theta=1 / 2)
$$


ДОКАЗАТЕЛЬСТВО ТЕОРЕМЫ 6 . Поскольку при $x \geqslant 2$ имеем $R_{0}(x)=-\mathscr{F}(x)+O(1)$, достаточно доказать, что

$$
\int_{x}^{2 x}|\mathscr{F}(u)| d u \geqslant 51 \cdot 10^{-4} x^{3 / 2} \quad(\forall x \geqslant 1) .
$$

В свою очередь, (12.4) вытекает из неравенства

$$
\int_{x}^{2 x}|\mathscr{F}(u)| u^{-3 / 2} d u \geqslant 51 \cdot 10^{-4} \quad(\forall x \geqslant 1) .
$$

Сделаем в интеграле замену переменного $u=e^{t}$. В результате этого, обозначив $X=\ln x+\frac{1}{2} \ln 2$ и учитьвая (11.4), придем к соотношению, эквивалентному (13.4):

$$
J(X)=\int_{X-4 \alpha}^{X+4 \alpha}|\varphi(t)| d t \geqslant 51 \cdot 10^{-4}, \quad X \geqslant \frac{1}{2} \ln 2 .
$$

Положим

$$
J_{1}(X)=\int_{X-4 \alpha}^{X+4 \alpha} \varphi(t) \widehat{g}(t-X) \exp \left\{i \gamma_{1}(X-t)\right\} d t .
$$

Используя результат леммы 3 , получаем оценку

$$
\left|J_{1}(X)\right| \leqslant J(X) \max _{y \in \mathbb{R}}|\widehat{g}(y)|=\frac{8}{3 \ln 2} \cdot J(X) .
$$

С другой стороны, интеграл $J_{1}(X)$ вычисляется в виде ряда, модуль которого не очень сложно оценить снизу. Имеем: ${ }^{4}$

$$
\begin{aligned}
\left|J_{1}(X)\right| & =\left|\int_{-4 \alpha}^{4 \alpha} \varphi(y+X) \widehat{g}(y) \exp \left(-i \gamma_{1} y\right) d y\right| \\
& =\left|\sum_{\substack{n=-\infty \\
n \neq 0}}^{+\infty} \frac{\exp \left(i \gamma_{n} X\right)}{\rho_{n}} \int_{-4 \alpha}^{4 \alpha} \widehat{g}(y) \exp \left\{i\left(\gamma_{n}-\gamma_{1}\right) y\right\} d y\right| \\
& =\left|\sum_{\substack{n=-\infty \\
n \neq 0}}^{+\infty} \frac{\exp \left(i \gamma_{n} X\right)}{\rho_{n}} g\left(\gamma_{n}-\gamma_{1}\right)\right| \\
& \geqslant \frac{|g(0)|}{\left|\rho_{1}\right|}-\sum_{n=2}^{\infty} \frac{\left|g\left(\gamma_{n}-\gamma_{1}\right)\right|}{\left|\rho_{n}\right|}-\sum_{n=-\infty}^{-1}\left|\frac{g\left(\gamma_{n}-\gamma_{1}\right)}{\rho_{n}}\right| \\
& =\frac{1}{\left|\rho_{1}\right|}-\sum_{n=2}^{\infty}\left|\frac{g\left(\gamma_{n}-\gamma_{1}\right)}{\rho_{n}}\right|-\sum_{n=1}^{\infty}\left|\frac{g\left(-\left(\gamma_{n}+\gamma_{1}\right)\right)}{\rho_{n}}\right| .
\end{aligned}
$$

$$
{ }^{4} \text { Равенство } \int_{a}^{b} \varphi(t) f(t) d t=\sum_{\substack{n=-\infty \\ n \neq 0}}^{+\infty} \rho_{n}^{-1} \int_{a}^{b} f(t) \exp \left(i \gamma_{n} t\right) d t \text { выполняется для любой функ- }
$$

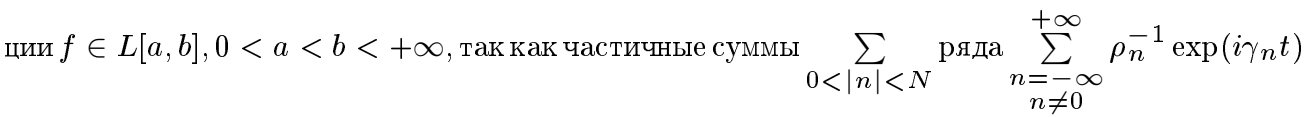
сходятся к $\varphi(t)$ в каждой точке отрезка $[a, b]$ и являются равномерно ограниченными на этом отрезке (см. [3, с. 122, (9)-(10)]). 
Ясно, что если доказать оценку

$$
\sigma=\frac{1}{\left|\rho_{1}\right|}-\sum_{n=2}^{\infty}\left|\frac{g\left(\gamma_{n}-\gamma_{1}\right)}{\rho_{n}}\right|-\sum_{n=1}^{\infty}\left|\frac{g\left(-\left(\gamma_{n}-\gamma_{1}\right)\right)}{\rho_{n}}\right|>0.02
$$

то из (15.4)-(17.4) получим (14.4):

$$
|J(X)| \geqslant \frac{3 \ln 2}{8}\left|J_{1}(X)\right| \geqslant \frac{3 \ln 2}{8} \sigma \geqslant \frac{3 \ln 2}{8 \cdot 50}>51 \cdot 10^{-4} .
$$

Тем самым теорема 6 будет доказана. Таким образом, осталось проверить справедливость неравенства (17.4).

Используя приведенные в [31] (см. также [27]) приближенные значения ординат первых шести нулей дзета-функции Римана, с помошью компьютера ${ }^{5}$ получаем оценки

$$
\frac{1}{\left|\rho_{1}\right|}>0.07, \quad \sum_{n=2}^{6}\left|\frac{g\left(\gamma_{n}-\gamma_{1}\right)}{\rho_{n}}\right|<0.026, \quad \sum_{n=1}^{3}\left|\frac{g\left(-\gamma_{n}-\gamma_{1}\right)}{\rho_{n}}\right|<0.002 .
$$

Теперь оценим сверху сумму

$$
\sigma_{1}=\sum_{n=7}^{\infty}\left|\frac{g\left(\gamma_{n}-\gamma_{1}\right)}{\rho_{n}}\right|+\sum_{n=4}^{\infty}\left|\frac{g\left(-\gamma_{n}-\gamma_{1}\right)}{\rho_{n}}\right| .
$$

При $n \geqslant 7$ имеем [31] $\gamma_{n}-\gamma_{1} \geqslant \gamma_{7}-\gamma_{1}>26$, а если $n \geqslant 4$, то $\gamma_{n}+\gamma_{1} \geqslant \gamma_{4}+\gamma_{1}>40$. Поэтому к оценке значений $\left|g\left(\gamma_{n}-\gamma_{1}\right)\right|$ и $\left|g\left(-\left(\gamma_{n}-\gamma_{1}\right)\right)\right|$ можно применить лемму 4 . Учитывая неравенство $14.1<\gamma_{1}<14.2$, из леммы 4 получаем

$$
\begin{aligned}
\sigma_{1} & \leqslant \sum_{n=7}^{\infty} \frac{0.8}{\left|\rho_{n}\right|\left(\gamma_{n}-\gamma_{1}+14.5\right)}+\sum_{n=4}^{\infty} \frac{0.8}{\left|\rho_{n}\right|\left(\gamma_{n}+\gamma_{1}-14\right)} \\
& <\sum_{n=7}^{\infty} \frac{0.8}{\left|\rho_{n}\right|\left(\gamma_{n}+0.3\right)}+\sum_{n=4}^{\infty} \frac{0.8}{\left|\rho_{n}\right|\left(\gamma_{n}+0.1\right)} \\
& <0.8\left(\sum_{n=7}^{\infty} \frac{1}{\left|\rho_{n}\right|^{2}}+\sum_{n=4}^{\infty} \frac{1}{\left|\rho_{n}\right|^{2}}\right) \\
& =0.8\left(\sum_{n=4}^{\infty} \frac{2}{\left|\rho_{n}\right|^{2}}-\left(\frac{1}{\left|\rho_{4}\right|^{2}}+\frac{1}{\left|\rho_{5}\right|^{2}}+\frac{1}{\left|\rho_{6}\right|^{2}}\right)\right)
\end{aligned}
$$

\footnotetext{
${ }^{5}$ Мы использовали известные неравенства $a_{n} \leqslant \gamma_{n} \leqslant b_{n}, 1 \leqslant n \leqslant 7$, где $a_{n}, b_{n}$ - десятичные дроби, $0<b_{b}-a_{n}<10^{-3}$. Далее, делалась оценка $\left|\frac{g\left(\gamma_{n}-\gamma_{1}\right)}{\rho_{n}}\right| \leqslant \frac{M_{n}}{a_{n}}$, где $M_{n}=\max _{t \in \delta_{n}}|g(t)|, \delta_{n}=\left[a_{n}-b_{1}, b_{n}-a_{1}\right]$. Получить границу сверху $M_{n}^{\prime} \geqslant M_{n}$, где $M_{n}^{\prime}$ - конечные десятичные дроби, мало отличающиеся от $M_{n}$, не представило труда. Далее было установлено, что $\sum_{n=2}^{6} M_{n}^{\prime} / a_{n} \leqslant 0.026$. Аналогично оценивались и другие конечные суммы, связанные с $\rho_{1}, \ldots, \rho_{6}$. На компьютере велись только точные вычисления с рациональными числами. Таким образом, все неравенства строго доказаны. Однако, выкладки слишком громоздки для того, чтобы приводить их здесь.
} 
Имеет место равенство [3, с. 91-92]

$$
\sum_{\rho} \frac{2 \beta}{\beta^{2}+\gamma^{2}}=2+C-\ln 4 \pi<0.0465
$$

где $C$ - постоянная Эйлера, $\beta=\operatorname{Re} \rho, \gamma=\operatorname{Im} \rho$. Если справедлива гипотеза Римана, то

$$
\sum_{\rho} \frac{2 \beta}{\beta^{2}+\gamma^{2}}=\sum_{\substack{n=-\infty \\ n \neq 0}}^{+\infty} \frac{1}{\left|\rho_{n}\right|^{2}}=2 \sum_{n=1}^{\infty}\left|\rho_{n}\right|^{-2}<0.0465
$$

Имеем также

$$
\begin{gathered}
2\left(\left|\rho_{1}\right|^{-2}+\left|\rho_{2}\right|^{-2}+\left|\rho_{3}\right|^{-2}\right)>0.0175 \\
\left|\rho_{4}\right|^{-2}+\left|\rho_{5}\right|^{-2}+\left|\rho_{6}\right|^{-2}>0.002
\end{gathered}
$$

Из (19.4)-(21.4) получаем $\sigma_{1}<0,022$. Отсюда и из (18.4) находим

$$
\begin{aligned}
\sigma & =\frac{1}{\left|\rho_{1}\right|}-\sum_{n=2}^{6}\left|\frac{g\left(\gamma_{n}-\gamma_{1}\right)}{\rho_{n}}\right|-\sum_{n=2}^{3}\left|\frac{g\left(-\gamma_{n}-\gamma_{1}\right)}{\rho_{n}}\right|-\sigma_{1} \\
& >0.07-0.026-0.002-0.022=0.02 .
\end{aligned}
$$

Мы получили (17.4), а это, как отмечалось вьше, доказывает теорему 6.

Докажем сразу же и теорему 9, поскольку метод ее доказательства очень близок к методу, применявшемуся только что при доказательстве теоремы 6.

Лемма 5. Предположим, что справедлива гипотеза Римана. Пусть $b>0$, $\varphi(t)=\sum_{n=-\infty}^{+\infty} \rho_{n}^{-1} \exp \left(i \gamma_{n} t\right), Y>b$. Тогда вьполняется неравенство

$$
\int_{Y-b}^{Y+b}|\varphi(t)| d t \geqslant \frac{4 b}{\pi\left|\rho_{1}\right|}-\frac{\pi}{b} \sum_{\substack{n=-\infty \\ n \neq 0, n \neq 1}}^{+\infty} \frac{\left|\cos \left(\left(\gamma_{n}-\gamma_{1}\right) b\right)\right|}{\left|\left(\gamma_{n}-\gamma_{1}\right)^{2}-(\pi / 2 b)^{2}\right|\left|\rho_{n}\right|}
$$

ДоКАЗАТЕЛЬСТВо. Положим

$$
g_{1}(y)=\cos \left(\frac{\pi y}{2 b}\right), \quad|y| \leqslant b .
$$

Тогда [32, с. 357] имеем

$$
\widehat{g}_{1}(z)=\int_{-b}^{b} \cos \left(\frac{\pi y}{2 b}\right) \exp (i z y) d y=\frac{4 \pi a \cos (b z)}{\pi^{2}-4 z^{2} b^{2}} .
$$

Следовательно,

$$
\left|\widehat{g}_{1}(z)\right|=\frac{\pi}{b}\left|\frac{\cos b z}{z^{2}-(\pi / 2 b)^{2}}\right|
$$


Из (22.4) и (23.4) находим

$$
\begin{aligned}
& \int_{Y-b}^{Y+b}|\varphi(t)| d t \geqslant\left|\int_{Y-b}^{Y+b} \varphi(t) g_{1}(Y-t) \exp \left(i \gamma_{1}(t-Y)\right) d t\right| \\
& =\left|\int_{-b}^{b} \varphi(Y+y) g_{1}(y) \exp \left(-i \gamma_{1} y\right) d y\right| \\
& =\left|\sum_{\substack{n=-\infty \\
n \neq 0}}^{+\infty} \rho_{n}^{-1} \int_{-b}^{b} \exp \left\{-i \gamma_{n}(Y+y)\right\} g_{1}(y) \exp \left(-i \gamma_{1} y\right) d y\right| \\
& =\left|\sum_{\substack{n=-\infty \\
n \neq 0}}^{+\infty} \frac{e^{i \gamma_{n} Y}}{\rho_{n}} \int_{-b}^{b} g_{1}(y) \exp \left\{i y\left(\gamma_{n}-\gamma_{1}\right)\right\} d y\right| \\
& =\left|\sum_{\substack{n=-\infty \\
n \neq 0}}^{+\infty} \frac{e^{i \gamma_{n} Y}}{\rho_{n}} \widehat{g}_{1}\left(\gamma_{n}-\gamma_{1}\right)\right| \\
& \geqslant\left|\frac{\widehat{g}_{1}(0)}{\rho_{1}}\right|-\sum_{\substack{n=-\infty \\
n \neq 0, n \neq 1}}^{+\infty}\left|\frac{\widehat{g}_{1}\left(\gamma_{n}-\gamma_{1}\right)}{\rho_{n}}\right| \\
& =\frac{4 b}{\pi\left|\rho_{1}\right|}-\frac{\pi}{b} \sum_{\substack{n=-\infty \\
n \neq 0, n \neq 1}}^{+\infty} \frac{\left|\cos \left(\left(\gamma_{n}-\gamma_{1}\right) b\right)\right|}{\left|\left(\gamma_{n}-\gamma_{1}\right)^{2}-(\pi / 2 b)^{2}\right|\left|\rho_{n}\right|} .
\end{aligned}
$$

Лемма 5 доказана.

ДОКАЗАТЕЛЬСТВО ТЕОРЕМЫ 9. ПоЛОЖим

$$
b=\frac{5 \pi}{\gamma_{1}}, \quad I(Y)=\int_{Y-b}^{Y+b} \varphi(t) d t, \quad J(Y)=\int_{Y-b}^{Y+b}|\varphi(t)| d t, \quad Y>b .
$$

Интеграл $I(Y)$ легко вычисляется:

$$
I(Y)=\sum_{\substack{n=-\infty \\ n \neq 0}}^{+\infty} \frac{2 e^{i \gamma_{n} Y} \sin \left(\gamma_{n} b\right)}{\rho_{n} \gamma_{n}}
$$

Учитывая выбор $b$, находим

$$
|I(Y)| \leqslant 4 \sum_{n=2}^{\infty} \frac{\left|\sin \left(5 \pi \gamma_{n} / \gamma_{1}\right)\right|}{\left|\rho_{n}\right| \gamma_{n}}
$$

К оценке снизу интеграла $J(Y)$ применим лемму 5. Получим

$$
J(Y) \geqslant \frac{20}{\left|\rho_{1}\right| \gamma_{1}}-\frac{\gamma_{1}}{5} \sum_{\substack{n=-\infty \\ n \neq 0, n \neq 1}}^{+\infty} \frac{\left|\cos \left(5 \pi \gamma_{n} / \gamma_{1}\right)\right|}{\left|\rho_{n}\right|\left|\left(\gamma_{n}-\gamma_{1}\right)^{2}-\left(\gamma_{1} / 10\right)^{2}\right|}
$$


Используя приближенное значение для $\gamma_{1}$ из [31], находим $\gamma_{1}^{2}<\gamma_{1}\left|\rho_{1}\right|<200$. Поэтому

$$
J(Y)>0.1-\frac{\gamma_{1}}{5} \sum_{\substack{n=-\infty \\ n \neq 0, n \neq 1}}^{+\infty} \frac{\left|\cos \left(5 \pi \gamma_{n} / \gamma_{1}\right)\right|}{\left|\rho_{n}\right| \mid\left(\gamma_{n}-\gamma_{1}\right)^{2}-2} .
$$

Легко проверяется справедливость неравенств

$$
\begin{gathered}
\left(\gamma_{n}-\gamma_{1}\right)^{2}-2>2 \gamma_{n} \quad \forall n \geqslant 2, \\
\left(\gamma_{n}-\gamma_{1}\right)^{2}-2=\left(\gamma_{-n}+\gamma_{1}\right)^{2}-2>\gamma_{n}^{2}>21\left|\gamma_{n}\right| \forall n \leqslant-2, \\
\frac{1}{5\left(\left(2 \gamma_{1}\right)^{2}-2\right)}<3 \cdot 10^{-4} .
\end{gathered}
$$

Соотношения (25.4) и (26.4) влекут за собой оценку

$$
\begin{aligned}
J(Y) & >0.1-\left(\frac{\gamma_{1}}{10}+\frac{\gamma_{1}}{105}\right) \sum_{n=2}^{\infty} \frac{\left|\cos \left(5 \pi \gamma_{n} / \gamma_{1}\right)\right|}{\left|\rho_{n}\right| \gamma_{n}}-3 \cdot 10^{-4} \\
& >0.0997-1.56 \sum_{n=2}^{\infty} \frac{\cos \left(5 \pi \gamma_{n} / \gamma_{1}\right)}{\left|\rho_{n}\right| \gamma_{n}}
\end{aligned}
$$

Поскольку при любых $x_{1}, x_{2}, \alpha \in \mathbb{R}$ выполняется неравенство $\left|x_{1} \cos \alpha+x_{2} \sin \alpha\right| \leqslant$ $\sqrt{x_{1}^{2}+x_{2}^{2}}$, из $(24.4)$ и $(27.4)$ находим

$$
J(Y)-|I(Y)|>0.0997-\sqrt{18.44} \sum_{n=2}^{\infty} \frac{1}{\left|\rho_{n}\right| \gamma_{n}} .
$$

При $n \geqslant 2$ имеем $\left|\rho_{n}\right| / \gamma_{n}=\sqrt{1+1 / 4 \gamma_{n}^{2}}<\sqrt{1+1 /\left(4 \cdot 21^{2}\right)}<\sqrt{1.001}$. Кроме того, $\left|\rho_{1}\right|^{-2}>0.00499$, а если и верна гипотеза Римана (см. доказательство предыдушей теоремы), то $\sum_{n=1}^{\infty}\left|\rho_{n}\right|^{-2}<0.02325$. Ввиду сказанного получаем

$$
\begin{aligned}
J(Y)-|I(Y)| & >0.0997-\sqrt{18.44} \sum_{n=2}^{\infty} \frac{\sqrt{1.001}}{\left|\rho_{n}\right|^{2}} \\
& >0.0997-4.3 \sum_{n=2}^{\infty}\left|\rho_{n}\right|^{-2} \\
& >0.0997-4.3(0.02325-0.00499)=0.021182 .
\end{aligned}
$$

Полученное неравенство показывает, что модуль интеграла от $\varphi(t)$ по отрезку $[Y-b, Y+b]$ менњше интеграла от $|\varphi(t)|$ на некоторую величину, отделенную от нуля. Отсюда заключаем, что интегралы от положительной и отрицательной части $\varphi(t)^{6}$ не слишком малы. На этой идее и построена завершаюшая часть доказательства теоремы 9 .

\footnotetext{
${ }^{6}$ Напомним, что ввиду симметричного расположения относительно $\mathbb{R}$ нулей $\zeta(s)$ функция $\varphi$ действительнозначна.
} 
Положим

$$
\begin{aligned}
\varphi^{+}(t) & =\max (0, \varphi(t)), & \varphi^{-}(t) & =-\min (0, \varphi(t)), \\
J^{+}(Y) & =\int_{Y-b}^{Y+b} \varphi^{+}(t) d t, & J^{-}(Y) & =\int_{Y-b}^{Y+b} \varphi^{-}(t) d t .
\end{aligned}
$$

Напомним, что $b=5 \pi / \gamma_{1}$. Имеем очевидные равенства

$$
\begin{gathered}
J(Y)=J^{+}(Y)+J^{-}(Y), \\
I(Y)=J^{+}(Y)-J^{-}(Y) .
\end{gathered}
$$

Из (29.4) можно выразить $J^{+}(Y)$ и $J^{-}(Y)$ :

$$
J^{+}(Y)=(J(Y)+I(Y)) / 2, \quad J^{-}(Y)=(J(Y)-I(Y)) / 2 .
$$

Из (30.4), (28.4) и элементарных неравенств $\left|x_{1}+x_{2}\right| \geqslant\left|x_{2}\right|-\left|x_{1}\right|,\left|x_{1}-x_{2}\right| \geqslant\left|x_{2}\right|-\left|x_{1}\right|$ находим

$$
J^{+}(Y)>0,0105, \quad J^{-}(Y)>0,0105 \quad \forall Y>b .
$$

Если верна гипотеза Римана, то $\varphi(t)=\mathscr{F}\left(e^{t}\right) e^{-t / 2}$. Поэтому при $x>1$

$$
\begin{aligned}
\int_{x}^{10 x} \mathscr{F}^{+}(u) d u & \geqslant \int_{x}^{10 x} \mathscr{F}^{+}(u)\left(\frac{x}{u}\right)^{3 / 2} d u \\
& =x^{3 / 2} \int_{\ln x}^{\ln 10+\ln x} \mathscr{F}^{+}\left(e^{t}\right) e^{-t / 2} d t \\
& =x^{3 / 2} \int_{Y-\frac{1}{2} \ln 10}^{Y+\frac{1}{2} \ln 10} \varphi^{+}(t) d t .
\end{aligned}
$$

(Здесь обозначено $Y=\frac{1}{2} \ln 10+\ln x$.) Поскольку

$$
\frac{1}{2} \ln 10>1.15, \quad b=\frac{5 \pi}{\gamma_{1}}<\frac{5 \cdot 3.142}{14.13}<1.12,
$$

из (31.4) и (32.4) выводим

$$
\int_{x}^{10 x} \mathscr{F}^{+}(u) d u>0.0105 x^{3 / 2} \quad \forall x>1 .
$$

Аналогично находим

$$
\int_{x}^{10 x} \mathscr{F}^{-}(u) d u>0.0105 x^{3 / 2} \quad \forall x>1 .
$$

Из определения функций $R_{0}(x)$ и $R(u)$ видно, что они не совпадают только лишш на счетном дискретном на $[1,+\infty)$ множестве точек. Следовательно, при любом $x \geqslant 1$ имеем

$$
\int_{x}^{10 x} R^{+}(u) d u=\int_{x}^{10 x} R_{0}^{+}(u) d u, \int_{x}^{10 x} R^{-}(u) d u=\int_{x}^{10 x} R_{0}^{-}(u) d u
$$


А при $u \geqslant 2$ вследствие (28.2) и (29.2) выполняются неравенства

$$
-\mathscr{F}(u) \geqslant R_{0}(u) \geqslant-\mathscr{F}(u)-\ln 2 \pi>-\mathscr{F}(u)-1.84 .
$$

Следовательно,

$$
\begin{aligned}
& R_{0}^{+}(u) \geqslant(\mathscr{F}(u))^{-}-1.84, \quad(\forall u \geqslant 2) \\
& R_{0}^{-}(u) \geqslant \mathscr{F}^{+}(u) .
\end{aligned}
$$

Из (33.4)-(36.4) находим, что в случае справедливостигипотезы Римана при всех $x \geqslant 2$ имеют место оценки

$$
\begin{aligned}
& \int_{x}^{10 x} R^{-}(u) d u=\int_{x}^{10 x} R_{0}^{-}(u) d u \geqslant \int_{x}^{10 x} \mathscr{F}^{+}(u) d u>0.0105 x^{3 / 2} \\
& \int_{x}^{10 x} R^{+}(u) d u=\int_{x}^{10 x} R_{0}^{+}(u) d u \geqslant \int_{x}^{10 x}\left(\mathscr{F}^{-}(u)-1.84\right) d u>0.0105 x^{3 / 2}-16.56 x .
\end{aligned}
$$

В частности, при всех $x \geqslant 2 \cdot 10^{9}$

$$
\int_{x}^{10 x} R^{+}(u) d u>\frac{x^{3 / 2}}{100}
$$

Теорема 9 доказана.

Перейдем к доказательствам оценок снизу интегралов от $|R(u)|, R^{+}(u), R^{-}(u)$ в предположении, что гипотеза Римана неверна. Из соотношений (23.3), (38.2) и леммы 2 следует упрощенная формула для $R(u)$ в случае $\Theta>1 / 2$ :

$$
R(u)=\mathscr{F}_{2}\left(\Theta_{1}, u\right)+o\left(u^{\Theta_{1}}\right) \quad(u \rightarrow+\infty) .
$$

(Мы обозначили $\Theta_{1}=1 / 4+\Theta / 2$.) Она убеждает нас в том, что теоремы 7 и 8 непосредственно вытекают из следуюшего утверждения.

Пусть $\widetilde{\rho}=\widetilde{\beta}+i \widetilde{\gamma}-$ произвольньй нуль $\zeta(s)$, вешественная часть которого $\widetilde{\beta}$ больше $\Theta_{1}$. Тогда для любого $a>1$ сушествует постоянная $c_{43}(a, \widetilde{\rho})>0$ такая, что при всех $x \geqslant 1$ вьполняется неравенство

$$
\int_{x}^{a x}\left|\mathscr{F}_{2}\left(\Theta_{1}, u\right)\right| d u \geqslant c_{43}(a, \widetilde{\rho}) x^{1+\widetilde{\beta}}
$$

Действительно, для доказательства теоремы 7 выберем нуль с вешественной частью $\widetilde{\beta}>\Theta-\varepsilon / 2$ (он сушествует по определению числа $\Theta$ ). А в доказательстве теоремы 8 возьмем $\widetilde{\rho}=\Theta+i \widetilde{\gamma}$.

Докажем сформулированное утверждение. Положим

$$
L(z)=\prod_{\substack{\operatorname{Re} \rho \geqslant \Theta_{1} \\ \rho \neq \tilde{\rho}}}(1-i z / \rho) .
$$


Поскольку количество нулей $\zeta(s)$ в прямоугольнике $\left\{\Theta_{1} \leqslant \operatorname{Re} s \leqslant \Theta,|\operatorname{Im} s| \leqslant T\right\}$ есть $O\left(T^{\delta_{1}}\right), 0<\delta_{1}<1$, то [33, с. 197] произведение (39.4) представляет из себя целую функцию, имеющую оценку роста

$$
|L(z)| \leqslant c_{44} \exp \left(c_{45}|z|^{\delta_{1}}\right) \quad \forall z \in \mathbb{C} .
$$

Нули $L(z)$ расположены в точности в точках $\{-i \rho\}, \operatorname{Re} \rho \geqslant \Theta_{1}, \rho \neq \widetilde{\rho}$. Постоянные $c_{44}, c_{45}$ и $\delta_{1}$, естественно, зависят от возможного значения $\Theta$.

Хорошо известно, что, каковы бы ни были заданные числа $\tau>0$ и $\delta \in(0,1)$, существует целая функция $f$, имеющая экспоненциальный тип, равньй $\tau$, и “достаточно быстро" убывающая на действительной оси:

$$
|f(t)|=O\left(\exp \left(-|t|^{\delta}\right)\right), \quad t \in \mathbb{R} .
$$

Такой целой функцией является, например,

$$
f(z)=\prod_{k=1}^{\infty}\left(\frac{\sin d_{k} t}{d_{k} t}\right), \quad d_{k}=\frac{\tau k^{-1 / \delta_{2}}}{\zeta\left(1 / \delta_{2}\right)}, \quad \delta_{2}=\frac{1+\delta}{2} .
$$

Возьмем такую функцию $f$ для $\tau=\frac{1}{2} \ln a$ и $\delta=\left(1+\delta_{1}\right) / 2$. Положим, наконец,

$$
g(z)=\left\{\begin{array}{l}
f(z) L(z), \quad \text { если } f(-i \widetilde{\rho}) \neq 0, \\
\frac{f(z) L(z)}{(z+i \widetilde{\rho})^{q}}, \text { если }-i \widetilde{\rho}-\text { нуль } f(z) \text { кратности } q .
\end{array}\right.
$$

Очевидно, что

$$
\begin{aligned}
& g(-i \rho)=0, \quad \operatorname{Re} \rho \geqslant \Theta_{1}, \rho \neq \widetilde{\rho}, \\
& g(-i \widetilde{\rho}) \neq 0 .
\end{aligned}
$$

Кроме того, из (40.4) и (41.4) вытекает, что $g$ является целой функцией экспоненциального типа $\tau$ и быстро убывает на действительной оси. Поэтому преобразование Фурье

$$
\widehat{g}(y)=\frac{1}{2 \pi} \int_{-\infty}^{+\infty} g(z) \exp (-i z y) d z
$$

лежит в классе $C^{\infty}(\mathbb{R})$ и имеет носитель внутри отрезка $[-\tau, \tau]=\left[-\frac{1}{2} \ln a, \frac{1}{2} \ln a\right]$.

Подготовительная работа завершена и теперь мы можем приступить к оценке снизу интеграла $\int_{x}^{a x}\left|\mathscr{F}_{2}\left(\Theta_{1}, u\right)\right| d u$. Сделаем замену переменного $u=e^{t}$ и обозначим $X=$ $\ln x+\frac{1}{2} \ln a$. Имеем:

$$
\begin{aligned}
\int_{x}^{a x}\left|\mathscr{F}_{2}\left(\Theta_{1}, u\right)\right| d u & \geqslant x \int_{x}^{a x}\left|\mathscr{F}_{2}\left(\Theta_{1}, u\right)\right| \frac{d u}{u} \\
& =x \int_{X-\tau}^{X+\tau}\left|\mathscr{F}_{2}\left(\Theta_{1}, e^{t}\right)\right| d t \\
& \geqslant \frac{x}{\max _{y \in \mathbb{R}}|\widehat{g}(y)|}\left|\int_{X-\tau}^{X+\tau} \mathscr{F}_{2}\left(\Theta_{1}, e^{t}\right) \widehat{g}(t-X) d t\right| \\
& =c_{46} x\left|\int_{X-\tau}^{X+\tau}\left(\sum_{\operatorname{Re} \rho \geqslant \Theta_{1}} \frac{\exp (\rho t)}{\rho}\right) \widehat{g}(t-X) d t\right|
\end{aligned}
$$


Сделав в последнем интеграле замену $t-X=y$, произведя почленное интегрирование (это возможно в силу равномерной сходимости ряда для $\mathscr{F}_{2}\left(\Theta_{1}, e^{t}\right)$ на любом отрезке в $\mathbb{R})$ и обозначив $\lambda=-i \rho$, получаем оценку

$$
\begin{aligned}
\int_{x}^{a x} \mid \mathscr{F}_{2}\left(\Theta_{1}, u\right) d u & \geqslant c_{46} x\left|\sum_{\operatorname{Re} \rho \geqslant \Theta_{1}} \frac{\exp (\rho X)}{\rho} \int_{-\tau}^{\tau} \widehat{g}(y) e^{i \lambda y} d y\right| \\
& =c_{46} x\left|\sum_{\operatorname{Re} \rho \geqslant \Theta_{1}} \frac{\exp (\rho X)}{\rho} g(\lambda)\right| .
\end{aligned}
$$

Ввиду соотношений (42.4) в последней сумме все слагаемые, кроме соответствуюших $\widetilde{\rho}$ (количество их равно $\nu$ - кратности нуля $\widetilde{\rho}$ ), обрашаются в ноль. Поэтому приходим к неравенствам

$$
\int_{x}^{a x}\left|\mathscr{F}_{2}\left(\Theta_{1}, u\right)\right| d u \geqslant c_{46} x\left|\frac{\nu}{\widetilde{\rho}} g(-i \rho)\right||\exp \{(\widetilde{\beta}+i \widetilde{\gamma}) X\}|=c_{47} x \exp (\widetilde{\beta} X)>c_{47} x^{1+\widetilde{\beta}}
$$

а это и доказьвает требуемое.

Для дальнейшего нам потребуется доказать существование еще одного вида непрерьвных на $\mathbb{R}$ функций с конечным носителем, которые вместе со своим преобразованием Фурье имеют нужные для наших целей специальные свойства. Рассмотрим семейство функций

$$
\begin{gathered}
f(\beta, \gamma, z)=\frac{1}{1+(z / \beta)^{2}} \cdot \frac{\operatorname{ch}\left(\frac{2 \pi \beta}{\gamma}\right)-\cos \left(\frac{2 \pi z}{\gamma}\right)}{\operatorname{ch}\left(\frac{2 \pi \beta}{\gamma}\right)-1}, \\
\beta, \gamma \in \mathbb{R}, \quad \frac{1}{2}<\beta<1, \quad \gamma>10, \quad z \in \mathbb{C} .
\end{gathered}
$$

Лемма 6. Относительно любой функиии вида (43.4) с ограничениями на параметры (44.4) справедливы следующие утверэсдения:

1) $f$ - четная целая функиия әкспоненииального типа $2 \pi / \gamma$, вещественная на $\mathbb{R}$. Все ее нули в $\mathbb{C}$ имеют вид $m \gamma \pm i \beta, m \in \mathbb{Z} \backslash\{0\}$.

2) $f(\beta, \gamma, 0)=1$.

3) $0<f(\beta, \gamma, z)<1 \quad \forall z \in \mathbb{R} \backslash\{0\}$.

4) Если $\gamma>2 \pi|z|$, то равномерно по $\beta, \gamma, z$

$$
f(\beta, \gamma, z)=1+O\left(\left(1+|z|^{2}\right) \gamma^{-2}\right) .
$$

5) Преобразование Фурье

$$
\widehat{f}(\beta, \gamma, y)=\frac{1}{2 \pi} \int_{-\infty}^{+\infty} f(\beta, \gamma, z) \exp (-i z y) d z
$$

положительно при всех $y \in(-2 \pi \gamma, 2 \pi \gamma)$ и обращается в нуль всюду вне этого интервала.

Проверка истинности утверждений 1) и 2) леммы 6 предоставляется читателю. Докажем утверждения 3)-5). Для вычисления преобразования Фурье функции $f$ воспользуемся известной формулой [34, с. 391]

$$
\int_{-\infty}^{+\infty} \frac{\cos b x}{x^{2}+1} d x=\pi e^{-|b|}, \quad b \in \mathbb{R}
$$


ДокАЗАТЕльство. Учитьвая четность функции $f$ и используя $(46.4)$, находим

$$
\begin{aligned}
\widehat{f}(\beta, \gamma, y)= & \frac{1}{2 \pi} \int_{-\infty}^{+\infty} \frac{\operatorname{ch}\left(\frac{2 \pi \beta}{\gamma}\right)-\cos \left(\frac{2 \pi z}{\gamma}\right)}{\operatorname{ch}\left(\frac{2 \pi \beta}{\gamma}\right)-1} \cdot \frac{\cos z y}{1+(z / \beta)^{2}} d z \\
= & \frac{\beta}{2 \pi\left(\operatorname{ch}\left(\frac{2 \pi \beta}{\gamma}\right)-1\right)} \\
& \times \int_{-\infty}^{+\infty} \frac{\cos (\beta x y) \operatorname{ch}\left(\frac{2 \pi \beta}{\gamma}\right)-\frac{1}{2} \cos \left(\beta x y+\frac{2 \pi \beta x}{\gamma}\right)-\frac{1}{2} \cos \left(\beta x y-\frac{2 \pi \beta x}{\gamma}\right)}{x^{2}+1} d x \\
= & \frac{\operatorname{ch}\left(\frac{2 \pi \beta}{\gamma}\right) \exp (-\beta|y|)-\frac{1}{2} \exp \left(-\beta\left|y+\frac{2 \pi}{\gamma}\right|\right)-\frac{1}{2} \exp \left(-\beta\left|y-\frac{2 \pi}{\gamma}\right|\right)}{2\left(\operatorname{ch}\left(\frac{2 \pi \beta}{\gamma}\right)-1\right)} .
\end{aligned}
$$

Раскрывая модули в полученном выражении и записывая стоящий в числителе дроби гиперболический косинус через экспоненты, приходим к равенствам

$$
\widehat{f}(\beta, \gamma, y)= \begin{cases}\frac{\operatorname{sh}\left(\left(\frac{2 \pi}{\gamma}-|y|\right)\right)}{2\left(\operatorname{ch}\left(\frac{2 \pi \beta}{\gamma}\right)-1\right)}, & |y|<\frac{2 \pi}{\gamma} \\ 0, & |y| \geqslant \frac{2 \pi}{\gamma}\end{cases}
$$

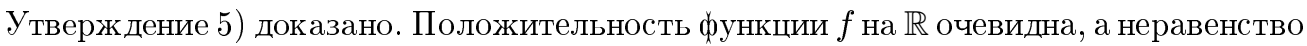
$f(\beta, \gamma, z)<1$ при любом действительном $z \neq 0$ легко следует из 2$)$, неотрицательности, непрерывности и четности на $\mathbb{R}$ преобразования Фурье $\widehat{f}(\beta, \gamma, y)$ :

$$
\begin{aligned}
f(\beta, \gamma, z) & =\int_{-\infty}^{+\infty} \widehat{f}(\beta, \gamma, y) \exp (i y z) d y=\int_{-\infty}^{+\infty} \widehat{f}(\beta, \gamma, y) \cos z y d y \\
& <\int_{-\infty}^{+\infty}|\widehat{f}(\beta, \gamma, y)| d y=\int_{-\infty}^{+\infty} \widehat{f}(\beta, \gamma, y) d y=f(\beta, \gamma, y)=1
\end{aligned}
$$

Тем самым установлено неравенство 3). Для доказательства 4) заменим в выражении для $f(\beta, \gamma, z)$ функции $\cos$ и сh по известным формулам: $\cos w=1-w^{2} / 2+O\left(w^{4}\right)$, $\operatorname{ch} w=1+w^{2} / 2+O\left(w^{4}\right)(w \geqslant 1)$. Тогда при $\gamma>2 \pi|z|, z \notin\left(K_{1} \cup K_{2}\right)\left(K_{1}=\{z \in \mathbb{C} \mid\right.$ $\left.|z-i \beta|<0,1\}, K_{2}=-K_{1}\right)$ получим

$$
f(\beta, \gamma, z)=\frac{\frac{1}{2}\left(\left(\frac{2 \pi \beta}{\gamma}\right)^{2}+\left(\frac{2 \pi z}{\gamma}\right)^{2}+O\left(\left(1+|z|^{4}\right) \gamma^{-4}\right)\right)}{\left(1+\left(\frac{z}{\beta}\right)\right)\left(\frac{1}{2}\left(\frac{2 \pi \beta}{\gamma}\right)^{2}+O\left(\gamma^{-4}\right)\right)}
$$

Разделив числитель и знаменатель этой дроби на $\frac{1}{2}\left(\frac{2 \pi \beta}{\gamma}\right)^{2}$, приходим к равенствам

$$
\begin{aligned}
f(\beta, \gamma, z) & =\frac{\left.1+(z / \beta)^{2}+O\left(\left(1+|z|^{4}\right) \gamma^{-2}\right)\right)}{\left(1+(z / \beta)^{2}\right)\left(1+O\left(\gamma^{-2}\right)\right)} \\
& =\left(1+O\left(\frac{1+|z|^{4}}{\gamma^{2}\left(1+(z / \beta)^{2}\right)}\right)\right)\left(1+O\left(\gamma^{-2}\right)\right)
\end{aligned}
$$


Отсюда сразу видно, что при $|z|<0.1$

$$
f(\beta, \gamma, z)=1+O\left(\gamma^{-2}\right),
$$

а при $0.1<|z|<\gamma / 2 \pi, z \notin\left(K_{1} \cup K_{2}\right)$

$$
f(\beta, \gamma, z)=1+O\left(z^{2} \gamma^{-2}\right) .
$$

В частности, из (47.4) следует, что на гранищах кругов $K_{1}$ и $K_{2}$ выполняется неравенство $|f(\beta, \gamma, z)-1| \leqslant c_{48} \gamma^{-2}$. Поскольку функция $f(\beta, \gamma, z)$ целая, такое же неравенство выполняется по принципу максимума и при $z \in\left(K_{1} \cup K_{2}\right)$. Тем самым (45.4) доказано при всех $|z|<\gamma / 2 \pi$. Лемма полностью доказана.

ДоКАЗАТЕЛЬСТВо ТЕОРЕМЫ 10. Возьмем произвольные $\varepsilon>0, a>1$ и зафиксируем их. Из плотностных теорем вытекает сходимость ряда $\sum_{\operatorname{Re} \rho \geqslant \Theta_{1}}|\operatorname{Im} \rho|^{-1}$, где $\Theta_{1}=1 / 4+\Theta / 2$. Следовательно,

$$
\lim _{T \rightarrow+\infty} \sum_{\substack{\operatorname{Re} \rho \geqslant \Theta_{1} \\|\operatorname{Im} \rho|>N}}|\operatorname{Im} \rho|^{-1}=0 .
$$

Но так как согласно посылке теоремы $10 \Theta$ недостижимо, то легко сообразить, что для любого фиксированного $T$ существует $\delta=\delta(T)>0$ такое, что прямоугольник $\{s \in \mathbb{C}|\operatorname{Re} s \geqslant \Theta-\delta(T),| \operatorname{Im} s \mid \leqslant T\}$ не содержит нулей $\zeta(s)$. Поэтому найдется такое $\delta=\delta(a)$, что

$$
\sum_{\operatorname{Re} \rho \geqslant \Theta-\delta}|\operatorname{Im} \rho|^{-1}<\frac{\ln a}{4 \pi} .
$$

При этом, естественно, можно взять $\delta<\varepsilon$.

Возьмем $\widetilde{\rho}=\widetilde{\beta}+i \widetilde{\gamma}-$ нуль $\zeta(s)$, лежаший в полуплоскости $\operatorname{Re} s>\Theta-\delta$ и имеющий среди таких нулей наименьшую положительную ординату (если их несколько, то выберем тот, у которого больше действительная часть). Теперь все различные нули $\zeta(s)$, лежашие в полосе $\operatorname{Re} s \geqslant \Theta-\delta$, за исключением двух $\widetilde{\beta} \pm i \widetilde{\gamma}$ занумеруем в последовательность $\left\{\omega_{n}\right\}, n \in \mathbb{Z}, n \neq 0$. Через $\nu_{n}$ обозначим кратность нуля $\omega_{n}$. Нули нумеруются, как обычно, в порядке возрастания их мнимых частей, а при равных мнимых частях - в произвольном порядке. Наконец, $\omega_{-n}=\bar{\omega}_{n} \forall n \in \mathbb{N}$. Номер “0” в нашей нумерации не задействован. Согласно введенным обозначениям имеем

$$
\mathscr{F}_{2}\left(\Theta-\delta, e^{t}\right)=\widetilde{\nu}\left(\frac{e^{(\widetilde{\beta}+i \widetilde{\gamma}) t}}{\widetilde{\beta}+i \widetilde{\gamma}}+\frac{e^{(\widetilde{\beta}-i \widetilde{\gamma}) t}}{\widetilde{\beta}-i \widetilde{\gamma}}\right)+\mathscr{F}_{3}\left(e^{t}\right)
$$

где $\widetilde{\nu}-$ кратность нуля $\widetilde{\rho}$, а

$$
\mathscr{F}_{3}(x)=\sum_{|n| \geqslant 1} \frac{\nu_{n} x^{\omega_{n}}}{\omega_{n}}
$$

Перед тем, как приступить к оценкам интегралов от $\mathscr{F}_{2}^{+}$и $\mathscr{F}_{2}^{-}$, докажем еще одно вспомогательное утверждение. 
ЛЕмма 7. Обозначим ${ }^{7}$

$$
\beta_{n}=\operatorname{Re} \omega_{n}, \quad \gamma_{n}=\operatorname{Im} \omega_{n}, \quad q=\sum_{n=1}^{\infty} \frac{1}{\gamma_{n}}
$$

Тогда существует неотрищательная непрерывная на $\mathbb{R}$ функиия $\widehat{g}$, обращающаяся в нуль вне интервала $(-2 \pi q, 2 \pi q)$, такая, что

$$
\int_{-2 \pi q}^{2 \pi q} e^{(\widetilde{\beta}+i \widetilde{\gamma}) t} \widehat{g}(t) d t \neq 0
$$

а при любом $Y \in \mathbb{R}$

$$
\int_{Y-2 \pi q}^{Y+2 \pi q} \mathscr{F}_{3}\left(e^{t}\right) \widehat{g}(t-Y) d t=0 .
$$

ДокАЗАТЕЛЬСТво. Положим

$$
g_{N}(z)=\prod_{n=1}^{N} f\left(\beta_{n}, \gamma_{n}, z\right) .
$$

В силу (45.4) последовательность функций $g_{N}(z)$ имеет предел

$$
g(z)=\lim _{N \rightarrow \infty} g_{N}(z)=\prod_{n=1}^{\infty} f\left(\beta_{n}, \gamma_{n}, z\right)
$$

причем сходимость на любом компакте в $\mathbb{C}$ равномерна. Следовательно (см. утверждение 1) леммы 6$), g(z)$ является целой четной вешественной на $\mathbb{R}$ функцией и все ее нули в $\mathbb{C}$ расположены в точках

$$
m \gamma_{n} \pm i \beta_{n}, \quad m, n \in \mathbb{Z}, \quad m n \neq 0
$$

Согласно выбору нуля $\widetilde{\rho}=\widetilde{\beta}+\widetilde{\gamma}$ нет другого нуля $\zeta(s)$ с положительной мнимой частью, меньшей $\widetilde{\gamma}$, и с вещественной частью $\widetilde{\beta}$. Поэтому если бы для каких-нибудь $m, n \in \mathbb{Z}$ имело место равенство $\widetilde{\gamma}-i \widetilde{\beta}=m \gamma_{n} \pm i \beta_{n}$, то мы получили бы $\widetilde{\gamma}=\gamma_{|n|}$, $\beta=\beta_{|n|}$, т.е. $\widetilde{\rho}=\omega_{|n|}$. Но все элементы $\left\{\omega_{n}\right\}$ отличны от $\tilde{\rho}$ по определению этой последовательности. Сказанное означает, что

$$
g(\widetilde{\gamma}-i \widetilde{\beta}) \neq 0
$$

Поскольку преобразование Фурье произведения двух функций является сверткой их преобразований Фурье, справедливо соотношение

$$
\widehat{g}_{N}(y)=\widehat{f}\left(\beta_{1}, \gamma_{1}, y\right) * \cdots * f\left(\beta_{N}, \gamma_{N}, y\right) .
$$

\footnotetext{
${ }^{7}$ В сумме $q$ участвуют нули $\zeta(s)$, вешественные части которых больше или равны $\Theta-\delta>1 / 2$. Следовательно, по плотностной теореме [21, с. 237] имеем $q<+\infty$.
} 
Поэтому (см. утверждение 5) леммы 6)

$$
\begin{aligned}
& \widehat{g}_{N}(y)>0, \quad|y|<2 \pi q_{N}, \\
& \widehat{g}_{N}(y)=0, \quad|y| \geqslant 2 \pi q_{N},
\end{aligned}
$$

где $q_{N}=\sum_{n=1}^{N} \gamma_{n}^{-1}$.

Из утверждения 3) леммы 6 следует, что все функции $g_{N}$ положительны на $\mathbb{R}$, и при любом фиксированном $z_{0} \in \mathbb{R} \backslash\{0\}$ последовательность $g_{N}\left(z_{0}\right)$ убьвает. Как отмечалось вьше, $\lim _{N \rightarrow \infty} g_{N}(z)=g(z) \quad \forall z \in \mathbb{Z}$. Кроме того, $g_{1}(z)=$ $f\left(\beta_{1}, \gamma_{1}, \cdot\right) \in L(\mathbb{R})$. Поэтому $g \in L(\mathbb{R})$,

$$
\lim _{N \rightarrow \infty} \int_{-\infty}^{+\infty}\left|g_{N}(z)-g(z)\right| d z=0
$$

Вследствие $(53.4) \widehat{g} \in C(\mathbb{R})$ и последовательность $\widehat{g}_{N}(y)$ сходится к $\widehat{g}(y)$ равномерно на $\mathbb{R}$. Это вместе с (52.4) влечет за собой справедливость соотношений

$$
\begin{gathered}
\widehat{g}(y) \geqslant 0 \quad \forall y \in \mathbb{R}, \\
\operatorname{supp} \widehat{g} \subset[-2 \pi q, 2 \pi q] .
\end{gathered}
$$

Первая часть леммы 7 доказана. Установим справедливость соотношений (49.4) и (50.4). По формуле обрашения преобразования Фурье имеем

$$
g(z)=\int_{-\infty}^{+\infty} \widehat{g}(y) \exp (i z y) d y
$$

Отсюда, из (51.4) и (54.4) находим

$$
\begin{aligned}
0 \neq g(\widetilde{\gamma}-i \widetilde{\beta}) & =\int_{-\infty}^{+\infty} \widehat{g}(y) \exp ((\widetilde{\beta}+i \widetilde{\gamma}) y) d y \\
& =\int_{-2 \pi q}^{2 \pi q} \widehat{g}(y) \exp ((\widetilde{\beta}+i \widetilde{\gamma}) y) d y
\end{aligned}
$$

Тем самым (49.4) доказано. Поскольку $g\left(\gamma_{n}-i \beta_{n}\right)=0$ при любых $n \in \mathbb{Z} \backslash\{0\}$,

$$
\int_{-2 \pi q}^{2 \pi q} \widehat{g}(y) \exp \left(\left(\beta_{n}+i \gamma_{n}\right) y\right) d y=0 \quad \forall n \in \mathbb{Z} \backslash\{0\} .
$$

Ряд для $\mathscr{F}_{3}\left(e^{t}\right)$ сходится равномерно на любом отрезке в $\mathbb{R}$ и, следовательно, допускает почленное интегрирование. Поэтому, учитьвая (55.4), находим

$$
\begin{aligned}
\int_{Y-2 \pi q}^{Y+2 \pi q} \mathscr{F}_{3}\left(e^{t}\right) \widehat{g}(t-Y) d t & =\int_{Y-2 \pi q}^{Y+2 \pi q}\left(\sum_{|n| \geqslant 1} \frac{\nu_{n} e^{\omega_{n} t}}{\omega_{n}}\right) \widehat{g}(t-Y) d t \\
& =\sum_{|n| \geqslant 1} \frac{\nu_{n} e^{\omega_{n} t}}{\omega_{n}} \int_{-2 \pi q}^{2 \pi q} e^{\omega_{n} y} \widehat{g}(y) d y=0 .
\end{aligned}
$$


Лемма 7 полностью доказана.

Продолжим доказательство теоремы. Обозначим $X=\ln x+\frac{1}{2} \ln a, \widehat{g}(\widetilde{\gamma}-i \widetilde{\beta})=\xi e^{i \alpha}$ (можно взять $\xi>0$, так как $\widehat{g}(\widetilde{\gamma}-i \widetilde{\beta}) \neq 0$ ). Если $Y$ пробегает некоторый отрезок действительной оси длины $2 \pi / \widetilde{\gamma}$, то точка $\exp (i Y \widetilde{\gamma})$ совершает полньй оборот по единичной окружности в $\mathbb{C}$. Поэтому найдутся два числа $X_{1}, X_{2} \in[X-\pi / \widetilde{\gamma}, X+\pi / \widetilde{\gamma}]$ такие, что

$$
\operatorname{Re}\left(\frac{\exp \left(i\left(X_{1} \widetilde{\gamma}+\alpha\right)\right)}{\widetilde{\rho}}\right)=\frac{1}{|\widetilde{\rho}|}, \quad \operatorname{Re}\left(\frac{\exp \left(i\left(X_{2} \widetilde{\gamma}+\alpha\right)\right)}{\widetilde{\rho}}\right)=-\frac{1}{|\widetilde{\rho}|} .
$$

Обозначим для краткости $\mathscr{F}_{2}(u)=\mathscr{F}_{2}(\Theta-\delta, u)$ и приступим к оценке снизу интеграла $\int_{x}^{a x} \mathscr{F}_{2}^{+}(u) d u$. Имеем:

$$
\int_{x}^{a x} \mathscr{F}_{2}^{+}(u) d u \geqslant x \int_{x}^{a x} \mathscr{F}_{2}^{+}(u) \frac{d u}{u}=x \int_{X-\frac{1}{2} \ln a}^{X+\frac{1}{2} \ln a} \mathscr{F}_{2}^{+}\left(e^{t}\right) d t .
$$

Поскольку

$$
\frac{1}{2} \ln a>2 \pi\left(\frac{1}{\widetilde{\gamma}}+\sum_{n=1}^{\infty} \frac{1}{\gamma_{n}}\right)=2 \pi q+2 \pi \widetilde{\gamma}^{-1},
$$

справедливы включения

$$
\left[X_{j}-2 \pi q, X_{j}+2 \pi q\right] \subset\left[X-\frac{1}{2} \ln a, X+\frac{1}{2} \ln a\right], \quad j=1,2 .
$$

Из (57.4) и (58.4) находим

$$
\begin{aligned}
\int_{x}^{a x} \mathscr{F}_{2}^{+}(u) d u & \geqslant \int_{X_{1}-2 \pi q}^{X_{1}+2 \pi q} \mathscr{F}_{2}^{+}\left(e^{t}\right) d t \\
& \geqslant \frac{x}{\max _{y \in \mathbb{R}} \widehat{g}(y)} \int_{X_{1}-2 \pi q}^{X_{1}+2 \pi q} \mathscr{F}_{2}^{+}\left(e^{t}\right) \widehat{g}\left(t-X_{1}\right) d t \\
& \geqslant c_{49} x \int_{X_{1}-2 \pi q}^{X_{1}+2 \pi q} \mathscr{F}_{2}\left(e^{t}\right) \widehat{g}\left(t-X_{1}\right) d t
\end{aligned}
$$

где $c_{49}=1 / \max _{y \in \mathbb{R}} \widehat{g}(y)$. Здесь мы использовали, что функция $\widehat{g}$ неотрицательна, непрерьвна на $\mathbb{R}$ и не равна нулю тождественно (см. (49.4)). Используя представление (48.4) функции $\mathscr{F}_{2}\left(e^{t}\right)$, а также равенства (50.4) и (49.4), получаем

$$
\begin{aligned}
& \int_{X_{1}-2 \pi q}^{X_{1}+2 \pi q} \mathscr{F}_{2}\left(e^{t}\right) \widehat{g}\left(t-X_{1}\right) d t \\
& =\int_{X_{1}-2 \pi q}^{X_{1}+2 \pi q}\left(\widetilde{\nu}\left(\frac{e^{(\widetilde{\beta}+i \widetilde{\gamma}) t}}{\widetilde{\beta}+i \widetilde{\gamma}}+\frac{e^{(\widetilde{\beta}-i \widetilde{\gamma}) t}}{\widetilde{\beta}-i \widetilde{\gamma}}\right)+\mathscr{F}_{3}\left(e^{t}\right)\right) \widehat{g}\left(t-X_{1}\right) d t \\
& =\widetilde{\nu} \int_{X_{1}-2 \pi q}^{X_{1}+2 \pi q}\left(\frac{e^{(\widetilde{\beta}+i \widetilde{\gamma}) t}}{\widetilde{\beta}+i \widetilde{\gamma}}+\frac{e^{(\widetilde{\beta}-i \widetilde{\gamma}) t}}{\widetilde{\beta}-i \widetilde{\gamma}}\right) \widehat{g}\left(t-X_{1}\right) d t \\
& =\widetilde{\nu} \int_{-2 \pi q}^{2 \pi q}\left(\frac{e^{(\widetilde{\beta}+i \widetilde{\gamma}) X_{1}}}{\widetilde{\beta}+i \widetilde{\gamma}} e^{(\widetilde{\beta}+i \widetilde{\gamma}) y} \widehat{g}(y)+\frac{e^{(\widetilde{\beta}-i \widetilde{\gamma}) X_{1}}}{\widetilde{\beta}-i \widetilde{\gamma}} e^{(\widetilde{\beta}+i \widetilde{\gamma}) y} \widehat{g}(y)\right) d y \\
& =\widetilde{\nu} e^{\beta X_{1}}\left(\frac{e^{i \widetilde{\gamma} X_{1}} g(\widetilde{\gamma}-i \widetilde{\beta})}{\widetilde{\beta}+i \widetilde{\gamma}}+\frac{e^{-i \widetilde{\gamma} X_{1}}}{\widetilde{\beta}-i \widetilde{\gamma}} g(-\widetilde{\gamma}-i \widetilde{\beta})\right) \text {. }
\end{aligned}
$$


Поскольку функция $g$ четна и вещественна на $\mathbb{R}$, числа $g(\widetilde{\gamma}-i \widetilde{\beta})$ и $g(-\widetilde{\gamma}-i \widetilde{\beta})$ являются комплексно-сопряженными и, следовательно, в скобках в последнем выражении стоит сумма двух комплексно-сопряженных чисел. Ввиду сказанного приходим к равенству

$$
\int_{X_{1}-2 \pi q}^{X_{1}+2 \pi q} \mathscr{F}_{2}^{+}\left(e^{t}\right) \widehat{g}\left(t-X_{1}\right) d t=2 \widetilde{\nu} e^{\beta X_{1}} \cdot \operatorname{Re}\left(\frac{e^{i \widetilde{\gamma} X_{1}} g(\widetilde{\gamma}-i \widetilde{\beta})}{\widetilde{\rho}}\right)
$$

Вспоминая обозначение $g(\widetilde{\gamma}-i \widetilde{\beta})=\xi e^{i \alpha}, \xi>0$, и исходя из выбора $X_{1}$, из (56.4), (59.4) и (60.4) находим

$$
\int_{x}^{a x} \mathscr{F}_{2}^{+}(u) d u \geqslant c_{50} x e^{\widetilde{\beta} X_{1}} \frac{\xi}{\left|\widetilde{\rho}_{1}\right|}=c_{51} x^{1+\widetilde{\beta}}>c_{51} x^{1+\Theta-\delta}
$$

С помошью совершенно аналогичных рассуждений получаем оценку для интеграла от $\mathscr{F}^{-}$:

$$
\begin{aligned}
\int_{x}^{a x} \mathscr{F}_{2}^{-}(u) d u & \geqslant x \int_{X-\frac{1}{2} \ln a}^{X+\frac{1}{2} \ln a} \mathscr{F}_{2}\left(e^{t}\right) d t \\
& \geqslant c_{49} x \int_{X_{2}-2 \pi q}^{X_{2}+2 \pi q} \mathscr{F}_{2}\left(e^{t}\right) \widehat{g}\left(t-X_{2}\right) d t \\
& \geqslant c_{49} x \int_{X_{2}-2 \pi q}^{X_{2}+2 \pi q}-\mathscr{F}_{2}\left(e^{t}\right) \widehat{g}\left(t-X_{2}\right) d t \\
& =-2 c_{49} x \widetilde{\nu} e^{\widetilde{\beta} X_{2}} \operatorname{Re}\left(\frac{e^{i \widetilde{\gamma} X_{2}} g(\widetilde{\gamma}-i \widetilde{\beta})}{\widetilde{\rho}}\right) \\
& =c_{51} x^{1+\widetilde{\beta}}>c_{51} x^{1+\Theta-\delta} .
\end{aligned}
$$

Из доказанных оценок и соотношения

$$
R(x)=-\mathscr{F}_{2}(\Theta-\delta, x)+o\left(x^{\Theta-\delta}\right) \quad(x \rightarrow+\infty)
$$

(см. лемму 2 и (38.2)) получаем

$$
\begin{aligned}
& \int_{x}^{A x} R^{+}(u) d u>c_{51} x^{1+\Theta-\delta}+o\left(x^{1+\Theta-\delta}\right), \\
& \int_{x}^{A x} R^{-}(u) d u>c_{51} x^{1+\Theta-\delta}+o\left(x^{1+\Theta-\delta}\right) .
\end{aligned}
$$

Так как $\delta<\varepsilon$, то отсюда при $x>x_{1}(\varepsilon)$ получаем неравенства (11.2). 
ДоКАЗАТЕЛЬСТво ТЕоРЕмы 11 основывается совершенно на той же идее, что и доказательство теоремы 10, только чуть-чуть проще. Вкратце, оно состоит в следующем.

По лемме 2

$$
R(u)=\mathscr{F}_{2}(\Theta, u)+o\left(u^{\Theta}\right) \quad(u \rightarrow+\infty) .
$$

Поэтому задача сводится к оценкам интегралов от $\mathscr{F}_{2}^{+}(\Theta, u)$ и $\mathscr{F}_{2}^{-}(\Theta, u)$. В качестве $\widetilde{\rho}$ берем нуль с наименьшей положительной ординатой, лежаший на прямой $\operatorname{Re} s=\Theta$ (в этой теореме предполагается, что $\Theta$ достижимо). Остальные различные нули $\zeta(s)$ на прямой $\operatorname{Re} s=\Theta$ расположим в последовательность $\omega_{n}$ по тому же принципу, что и ранее. Далее делаем в точности те же выкладки, что и в доказательстве теоремы 10. Но так как здесь $\widetilde{\beta}=\Theta$, то никакого $\varepsilon$ не появляется.

ДоКАЗАТЕльство теоРемы 13. Если справедлива гипотеза Римана, то из (1), леммы 1 и (35.2) получается следуюшая приближенная явная формула для $P(u)$ :

$$
P(u)=-(\ln u)^{-1}\left(\sqrt{u}+\sum_{\rho} \frac{u^{\rho}}{\rho}\right)+O\left(\frac{\sqrt{u}}{\ln ^{2} u}\right), \quad u \geqslant 2 .
$$

Интегрируя (61.4) от 2 до $x$, находим

$$
\begin{aligned}
\int_{2}^{x} P(u) d u & =-\left(\left.\frac{2}{3} \frac{u^{3 / 2}}{\ln u}\right|_{2} ^{x}+\frac{2}{3} \int_{2}^{x} \frac{\sqrt{u}}{\ln ^{2} u} d u\right. \\
& \left.+\sum_{\rho}\left(\left.\frac{u^{\rho+1}}{\rho(\rho+1) \ln u}\right|_{2} ^{x}+\frac{1}{\rho(\rho+1)} \int_{2}^{x} \frac{u^{\rho} d u}{\ln ^{2} u}\right)\right)+O\left(\int_{2}^{x} \frac{\sqrt{u} d u}{\ln ^{2} u}\right) .
\end{aligned}
$$

Поскольку $\rho=1 / 2+i \gamma, \int_{2}^{x} \sqrt{u} \ln ^{-2} u d u=O\left(x^{3 / 2} \ln ^{-2} x\right)$ и

$$
\sum_{\rho} \frac{1}{|\rho(\rho+1)|}=\sum_{\rho} \frac{1}{\sqrt{\gamma^{4}+2.5 \gamma^{2}+9 / 16}}=\omega \text { при } \Theta=1 / 2
$$

из (62.4) и (63.4) получаем

$$
\int_{2}^{x} P(u) d u=-\frac{2}{3} \frac{x^{3 / 2}}{\ln x}-\sum_{\rho} \frac{x^{3 / 2+i \gamma}}{\rho(\rho+1) \ln x}+O\left(\frac{x^{3 / 2}}{\ln ^{2} x}\right) .
$$

Отсюда и из определения функции $\mathscr{P}$ находим

$$
\mathscr{P}(x)=-\frac{2}{3}-\sum_{\rho} \frac{x^{i \gamma}}{\rho(\rho+1)}+O\left((\ln x)^{-1}\right)
$$

Из (63.4) и (64.4) легко следуют требуемые оценки $\bar{L}$ и $\underline{L}$. Неравенство $\underline{L} \neq \bar{L}$ вытекает из того, что функция $v(t)=\sum_{\rho} \frac{\exp (i \gamma t)}{\rho(\rho+1)}$ не имеет предела при $t \rightarrow+\infty$. 
Это - хорошо известньй факт из теории почти периодических функций. Впрочем, его легко доказать. Предположив, что сушествует

$$
l=\lim _{t \rightarrow+\infty} v(t),
$$

мы получили бы $l=0$, так как ввиду того, что все показатели $\gamma$ отличны от нуля и ряд (63.4) абсолютно сходится, справедливо соотношение

$$
\int_{0}^{T} v(t) d t=\sum_{\rho} \frac{e^{i \gamma t}-1}{i \gamma \rho(\rho+1)}=O(1) .
$$

С другой стороны,

$$
\int_{0}^{T} v(t) e^{-i \gamma_{1} t} d t=\frac{T}{\rho_{1}\left(\rho_{1}+1\right)}+O(1),
$$

но если $l=0$, то $\int_{0}^{T}|v(t)| d t=o(T)(T \rightarrow \infty)$. Полученное противоречие показьвает, что предел (65.4) не сушествует. Теорема 13 доказана.

Для доказательства теорем 14, 15, 17 и 19 нам потребуются два вспомогательных утверждения. Обозначим в предположении гипотезы Римана

$$
S(y, h)=\sum_{\gamma>0} \frac{\sin \gamma y}{\gamma} \frac{\sin \gamma h}{\gamma h}, \quad y \in \mathbb{R}, \quad h>0 .
$$

Ввиду (36.2) ряд (66.4) при всех $y \in \mathbb{R}$ и $h>0$ сходится абсолютно и равномерно по $y$ при фиксированном $h$. Из дальнейшего будет видно, что равномерная сходимость ряда (66.4) по совокупности переменных $(y, h)$ не имеет места.

ЛЕмма 8. Если верна гипотеза Римана, то равномерно по $x \geqslant 6$ u $h \in(0,1)$

$$
\frac{1}{2 h x^{3 / 2}} \int_{x \exp (-h)}^{x \exp h} R(u) d u=-2 S(\ln x, h)+w(x, h),
$$

$|w(x, h)| \leqslant c_{52}$, постоянная $c_{52}-$ абсолютная и әффективная.

ДокАЗАТЕльство. Ввиду ограничений на $x$ и $h$ в интеграле (67.4) $u \geqslant 2$. Поэтому из $(35.2)$ и (38.2) находим

$$
\begin{aligned}
& \frac{x^{-3 / 2}}{2 h} \int_{x \exp (-h)}^{x \exp h} R(u) d u \\
& =\frac{x^{-3 / 2}}{2 h} \int_{x \exp (-h)}^{x \exp h}\left(-\sum_{\gamma} \frac{u^{1 / 2+i \gamma}}{1 / 2+i \gamma}+O(1)\right) d u \\
& =\frac{x^{-3 / 2}}{2 h}\left(-\left.\sum_{\gamma} \frac{u^{3 / 2+i \gamma}}{(1 / 2+i \gamma)(3 / 2+i \gamma)}\right|_{x \exp (-h)} ^{x \exp h}+O(x \operatorname{sh} h)\right) \\
& =\frac{1}{2 h} \sum_{\gamma} \frac{x^{i \gamma}\left(e^{(3 / 2+i \gamma) h}-e^{-(3 / 2+i \gamma) h}\right)}{(\gamma+3 / 2 i)(\gamma+1 / 2 i)}+O(1 / \sqrt{x}) \\
& =\frac{1}{h} \sum_{\gamma} \frac{x^{i \gamma} \operatorname{sh}((3 / 2+i \gamma) h)}{\gamma^{2}-2 i \gamma-3 / 4}+O\left(x^{-1 / 2}\right) .
\end{aligned}
$$


Поскольку при $u, v \in \mathbb{R}$

$$
\operatorname{sh}(u+i v)=\operatorname{sh} u \cos v+i \sin v \operatorname{ch} u
$$

а при $\gamma \rightarrow \pm \infty$

$$
\left(\gamma^{2}-2 i \gamma-3 / 4\right)^{-1}=\gamma^{-2}+O\left(\gamma^{-3}\right)
$$

из (68.4) получаем

$$
\begin{aligned}
& \frac{x^{-3 / 2}}{2 h} \int_{x \exp (-h)}^{x \exp h} R(u) d u \\
& =\frac{\operatorname{sh}(3 h / 2)}{h} \sum_{\gamma} \frac{\cos (\gamma h) x^{i \gamma}}{\gamma^{2}-2 i \gamma-3 / 4} \\
& \quad+\frac{i \operatorname{ch}(3 h / 2)}{h} \sum_{\gamma}\left(\gamma^{-2}+O\left(\gamma^{-3}\right)\right) \sin (\gamma h) x^{i \gamma}+O\left(x^{-1 / 2}\right) \\
& =i \sum_{\gamma} \frac{x^{i \gamma}}{\gamma} \frac{\sin \gamma h}{\gamma h}+O(1) .
\end{aligned}
$$

Мы воспользовались ограниченностью $\frac{\operatorname{sh}(3 h / 2)}{h}$ и $\frac{\operatorname{ch}(3 h / 2)-1}{h}$ на $(0,1)$ и равномерной ограниченностью по $x$ и $h$ суммы ряда

$$
\sum_{\gamma}\left|\frac{x^{i \gamma} \sin \gamma h}{\gamma^{3} h}\right| \leqslant \sum_{\gamma} \frac{1}{\gamma^{2}}<+\infty
$$

Теперь обозначим $y=\ln x$ и в ряде

$$
\sum_{\gamma} \frac{x^{i \gamma}}{\gamma} \cdot \frac{\sin \gamma h}{\gamma h}
$$

перейдем к суммированию по $\gamma>0$. Имеем:

$$
\sum_{\gamma} \frac{x^{i \gamma}}{\gamma} \cdot \frac{\sin \gamma h}{\gamma h}=\sum_{\gamma>0} \frac{\sin \gamma h}{\gamma h}\left(\frac{e^{i \gamma y}}{\gamma}+\frac{e^{-i \gamma y}}{-\gamma}\right)=\sum_{\gamma} \frac{2 i \sin \gamma y}{\gamma} \cdot \frac{\sin \gamma h}{\gamma h}
$$

Из (69.4) и (70.4) получаем утверждение леммы 8.

Лемма 9. Предположим, что верна гипотеза Римана. Тогда существует әффективная абсолютная постоянная $c_{53} \in(0,0.1)$ такая, что при любых $Y \geqslant 1$ $u h \in\left(0, c_{53}\right]$ найдутся точки $y_{1}, y_{2} \in\left[Y, Y \exp \left(h^{-1}(\ln (1 / h))^{5 / 2}\right)\right]$, в которых ви-полняются неравенства

$$
\begin{aligned}
& S\left(y_{1}, h\right)>0.1 \ln (1 / h), \\
& S\left(y_{2}, h\right)<0.1 \ln h .
\end{aligned}
$$


ДОКАЗАТЕЛЬСТВО. Положим

$$
T(h)=h^{-1} \ln (1 / h), \quad S_{1}(y, h)=\sum_{0<\gamma \leqslant T(h)} \frac{\sin \gamma y}{\gamma} \cdot \frac{\sin \gamma h}{\gamma h} .
$$

Ввиду (29.2) имеем

$$
\sum_{\gamma>T} \gamma^{-2}=O\left(T^{-1} \ln T\right), \quad T \geqslant 2 .
$$

Поэтому равномерно по $y \in \mathbb{R}, h \in(0,1 / 3)$ с эффективной постоянной в символе $O$ вьполняется соотношение

$$
S(y, h)=S_{1}(y, h)+O(1)
$$

Используя (36.2), находим

$$
\begin{aligned}
S_{1}(h, h)= & h \sum_{0<\gamma \leqslant T(h)}\left(\frac{\sin \gamma h}{\gamma h}\right)^{2} \geqslant h \sum_{0<\gamma \leqslant \pi / 2 h}\left(\frac{\sin \gamma h}{\gamma h}\right)^{2} \\
\geqslant & h\left(\frac{2}{\pi}\right)^{2} \sum_{0<\gamma \leqslant \pi / 2 h} 1=\frac{4 h}{\pi^{2}} \mathscr{N}\left(\frac{\pi}{2 h}\right) \\
= & \frac{4 h}{\pi^{2}}\left(\frac{1}{2 h} \cdot \frac{\pi}{2 h} \ln \frac{\pi}{2 h}+O\left(\frac{1}{h}\right)\right)=\pi^{-2} \ln (1 / h)+O(1), \\
& S_{1}(-h, h)=-S_{1}(h, h) \leqslant \pi^{-2} \ln h+O(1) .
\end{aligned}
$$

Согласно аппроксимационной теореме Дирихле $[15$, с. 133] для любого $X \in \mathbb{N}$ найдется натуральное число $k, 1 \leqslant k \leqslant X^{N}, N=\mathscr{N}(T(h))$, такое, что при всех $m=1,2, \ldots, N$ выполнены неравенства

$$
\left\|\frac{\gamma_{m} Y}{\pi} k\right\|<X^{-1}
$$

(Здесь мы занумеровали нули $\zeta(s)$ так же, как и в доказательстве теоремы 6 , а через $\|z\|$ обозначим расстояние от $z$ до ближайшего целого числа.) Положим $X=\left[\ln ^{2} h\right]$,

$$
y_{1}=2 k Y+h, \quad y_{2}=2 k Y-h,
$$

где $k \in \mathbb{N}$ есть то самое число, для которого справедливы неравенства (73.4). Так как $Y \geqslant 1,0<h<1 / 3$, то очевидно, что $y_{j} \geqslant Y, j=1,2$. С другой стороны, $y_{j} \leqslant 3 X^{N} Y$, откуда имеем, что

$y_{j} Y^{-1} \leqslant 3 \exp \{\mathscr{N}(T(h)) \ln X\} \leqslant 3 \exp \left\{c_{54} h^{-1} \ln ^{2} h \ln |\ln h|\right\}<\exp \left\{h^{-1}(\ln (1 / h))^{5 / 2}\right\}$

при $h \in\left(0, c_{55}\right), j=1,2$. 
Таким образом, для доказательства леммы 9 осталось лишь установить справедливость соотношений (71.4) для $y_{j}$, определенных в (74.4), $j=1,2$, при $0<h<$ $c_{53}<c_{55}$. Для $j=1,2$ имеем:

$$
\begin{aligned}
& \left|S_{1}\left(y_{j}, h\right)-S_{1}\left(y_{j}-2 k Y, h\right)\right| \\
& \quad=\left|\sum_{0<\gamma<T(h)} \frac{\left(\sin \gamma y_{j}-\sin \left(\left(y_{j}-2 k Y\right) \gamma\right)\right)}{\gamma} \cdot \frac{\sin \gamma h}{\gamma h}\right| \\
& \quad=\left|\sum_{m=1}^{N} \frac{2 \sin \left(\left(y_{j}-k Y\right) \gamma_{m}\right) \sin \left(k Y \gamma_{m}\right)}{\gamma_{m}} \cdot \frac{\sin \gamma_{m} h}{\gamma_{m} h}\right| \\
& \quad \leqslant \sum_{m=1}^{N}\left|\frac{2 \sin \left(k Y \gamma_{m}\right)}{\gamma_{m}}\right| .
\end{aligned}
$$

Так как $|\sin \pi \alpha| \leqslant \pi\|\alpha\|(\forall \alpha \in \mathbb{R})$, то, учитывая (73.4) при $1 \leqslant m \leqslant N$, получаем неравенства

$$
\sin \left(k Y \gamma_{m}\right) \mid \leqslant \pi\left\|\frac{k Y \gamma_{m}}{\pi}\right\| \leqslant \pi\left(\left[\ln ^{2} h\right]\right)^{-1}=O\left(\ln ^{-2} h\right)
$$

Из $(75.4),(76.4)$ и $(36.3)$ находим

$$
\left|S_{1}\left(y_{j}, h\right)-S_{1}\left(y_{j}-2 k Y, h\right)\right| \leqslant c_{56} \ln ^{-2} h \sum_{m=1}^{N} \gamma_{m}^{-1}<c_{56}, \quad j=1,2 .
$$

Ввиду (74.4) неравенства (77.4) можно переписать следующим образом:

$$
\begin{array}{r}
\left|S_{1}\left(y_{1}, h\right)-S_{1}(h, h)\right|<c_{57}, \\
\left|S_{1}\left(y_{2}, h\right)-S_{1}(-h, h)\right|<c_{57} .
\end{array}
$$

Полученные вьше оценки для $S_{1}( \pm h, h)$ вместе с $(72.4)$ и (78.4) влекут за собой справедливость неравенств $(71.4)$ при $h \in\left(0, c_{53}\right)$. Лемма 9 доказана.

ДОКАЗАТЕЛЬСТВО ТЕОРЕМЫ 14. ПоскольКу При Любом Постоянном $a>1$ и $u \in[x, a x]$ имеем

$$
(\ln u)^{-1}=(\ln x)^{-1}+O_{a}\left((\ln x)^{-2}\right)
$$

из (61.4) и (2.2) находим

$$
\int_{x}^{a x} P(u) d u=-(\ln x)^{-1} \int_{x}^{a x}\left(\sqrt{u}+\sum_{\rho} u^{\rho} / \rho\right) d u+O\left(x^{3 / 2}(\ln x)^{-2}\right) .
$$


Вычисляя интеграл, стоящий в правой части (79.4), приходим к соотношениям

$$
\begin{array}{rl}
\int_{x}^{a x} & P(u) d u \\
= & -(\ln x)^{-1}\left(\frac{2}{3}\left((a x)^{3 / 2}-x^{3 / 2}\right)+\sum_{\rho} \frac{(a x)^{\rho+1}-x^{\rho+1}}{\rho(\rho+1)}\right)+O\left(x^{3 / 2}(\ln x)^{-2}\right) \\
= & \frac{x^{3 / 2}}{\ln x}\left(-\frac{2}{3}\left(a^{3 / 2}-1\right)-\sum_{\rho} \frac{x^{i \gamma}\left(a^{3 / 2+i \gamma}-1\right)}{\rho(\rho+1)}\right)+O\left(x^{3 / 2}(\ln x)^{-2}\right) \\
\leqslant & \frac{x^{3 / 2}}{\ln x}\left(-\frac{2}{3}\left(a^{3 / 2}-1\right)+\sum_{\rho} \frac{a^{3 / 2}+1}{|\rho(\rho+1)|}\right)+O\left(x^{3 / 2}(\ln x)^{-2}\right) \\
= & -\frac{2}{3} \frac{x^{3 / 2}\left(a^{3 / 2}-1\right)}{\ln x} K(a)+O\left(x^{3 / 2}(\ln x)^{-2}\right) .
\end{array}
$$

Первая часть теоремы 14 доказана. Приступим к доказательству второй части. Положим

$$
h=\frac{1}{2} \ln (1+\alpha), \quad Y=h+\ln X
$$

Ясно, что $\alpha / 3<h<\alpha / 2$ при всех $\alpha \in(0,1 / 2)$ и при $0<\alpha<\min \left(e^{-50}, c_{53}\right)$ согласно лемме 9 на отрезке 8

$$
\left[Y, Y \exp \left\{h^{-1}(\ln (1 / h))^{2.5}\right\}\right] \subset\left[Y, Y \exp \left\{\frac{1}{2} \alpha^{-1} \ln ^{3}(1 / \alpha)\right\}\right]
$$

найдется точка $y_{2}$, для которой

$$
S\left(y_{2}, h\right)<0.1 \ln h<0.1 \ln \alpha .
$$

По лемме 8 это означает, что справедливо неравенство $\left(x=\exp y_{2}\right)$

$$
\frac{x^{-3 / 2}}{2 h} \int_{x \exp (-h)}^{x \exp h} R(u) d u>0.2 \ln (1 / \alpha)+O(1),
$$

т.е. при малых $\alpha$ верна оценка

$$
\int_{x \exp (-h)}^{x \exp h} R(u) d u>0.2 \alpha x^{3 / 2} \ln (1 / \alpha)-c_{58} \alpha x^{3 / 2}
$$

с абсолютной и эффективной постоянной $c_{57}$.

Ввиду (1) и леммы 1

$$
P(u)=\frac{R(u)}{\ln u}+O\left(\frac{\sqrt{u}}{\ln u}\right) \quad(\Theta=1 / 2, u \geqslant 2) .
$$

\footnotetext{
8 Это включение заведомо имеет место, если $\alpha<e^{-50}$.
} 
Обозначая $\xi=x e^{-h}$ (тогда $x e^{h}=\xi+\xi \alpha$ ), из (81.4) и (82.4) находим (постоянные в символах $O$ абсолютные)

$$
\begin{aligned}
\int_{\xi}^{\xi+\xi \alpha} & P(u) d u \\
& =\int_{\xi}^{\xi+\xi \alpha} \frac{R(u)}{\ln u} d u+O\left(\frac{\alpha \xi^{3 / 2}}{\ln \xi}\right) \\
& =\frac{1}{\ln \xi} \int_{\xi}^{\xi+\xi \alpha} R(u) d u+\int_{\xi}^{\xi+\xi \alpha} R(u)\left(\frac{1}{\ln u}-\frac{1}{\ln \xi}\right) d u+O\left(\frac{\alpha \xi^{3 / 2}}{\ln \xi}\right) \\
& >\frac{0.2 \alpha \xi^{3 / 2} \ln (1 / \alpha)}{\ln \xi}-\frac{c_{59} \alpha \xi^{3 / 2}}{\ln \xi}-\left[\max _{u \in[\xi, \xi+\alpha]}\left(\frac{1}{\ln \xi}-\frac{1}{\ln u}\right)\right] \cdot \int_{\xi}^{\xi+\xi \alpha}|R(u)| d u \\
& >\frac{0.2 \alpha \xi^{3 / 2} \ln (1 / \alpha)}{\ln \xi}-\frac{c_{60} \alpha \xi^{3 / 2}}{\ln \xi}>\frac{\alpha \xi^{3 / 2} \ln (1 / \alpha)}{6 \ln \xi}
\end{aligned}
$$

при $\alpha \in\left(0, c_{24}\right)$. Тем самьп неравенство (18.2) доказано. Осталось установить, что $\xi \in\left[X, X^{B}\right]$, где $B=\exp \left(\alpha^{-1} \ln ^{3}(1 / \alpha)\right)$. Имеем: $\ln \xi=\ln x-h=y_{2}-h \geqslant$ $Y-h=\ln X$. Требуемая оценка снизу для $\xi$ получена. С другой стороны, $\ln \xi=y_{2}-h<Y B / 2=B(h+\ln X) / 2<B \ln X$. Поэтому $\xi<X^{B}$. Вторая часть теоремы 14 доказана.

Перейдем к доказательству третьей части теоремы 14. В доказательстве леммы 9 было установлено предельное соотношение: $\lim _{h \rightarrow+0} S(-h, h)=-\infty$. Поэтому найдется $h_{0} \in(0,0.01)$, для которого выполняется неравенство

$$
S\left(-h_{0}, h_{0}\right)<1-c_{52}
$$

где $c_{52}$ - постоянная леммы 8. Поскольку ряд по системе $\left\{\sin \gamma_{n} y\right\}_{n=1}^{\infty}$ для функции $S\left(y, h_{0}\right)$ абсолютно сходится, $S\left(y, h_{0}\right)$ является почти периодической функцией Бора. Следовательно, для любого $\varepsilon>0$ множество $\varepsilon$-почти периодов $S\left(y, h_{0}\right)$ относительно плотно [30]. Это означает, что для любого $\varepsilon>0$ существует такое число $T(\varepsilon)$, что на любом отрезке в $\mathbb{R}$ длины $T(\varepsilon)$ имеется число $\tau$, для которого

$$
\sup _{y \in \mathbb{R}}\left|S\left(y+\tau, h_{0}\right)-S\left(y, h_{0}\right)\right|<\varepsilon .
$$

Из сказанного несложно вывести, что при некотором достаточно большом $L$ и при любом $\alpha>0$ на отрезке $[\alpha, \alpha+L]$ отышется точка $\widehat{y}=\widehat{y}(\alpha, L)$ такая, что

$$
S\left(\widehat{y}, h_{0}\right)<-0.9-c_{52}
$$

(Достаточно взять $\varepsilon=0,1, L=T(0,1)$ и, положив $y=-h_{0}$, применить сформулированную вьше теорему об $\varepsilon$-почти периодах к отрезку $\left[\alpha+h_{0}, \alpha+L+h_{0}\right]$.) Тогда по лемме 8 получаем, что при $\alpha_{1} \geqslant 6$ на отрезке $\left[\alpha_{1}, \alpha_{1} L_{1}\right]\left(L_{1}=\exp L, \alpha_{1}=\exp \alpha\right)$ найдется точка $z$, для которой имеет место неравенство

$$
\int_{z \exp \left(-h_{0}\right)}^{z \exp h_{0}} R(u) d u>3.6 h_{0} z^{3 / 2} .
$$


Так как ${ }^{9}$

$$
\int_{z \exp \left(-h_{0}\right)}^{z \exp h_{0}} \sqrt{u} d u=(4 / 3) z^{3 / 2} \operatorname{sh}\left(3 h_{0} / 2\right)<(7 / 3) z^{3 / 2} h_{0},
$$

то, воспользовавшись приближенной явной формулой (61.4) для $P(u)(\Theta=1 / 2)$ и (38.2), получаем

$$
\int_{z \exp \left(-h_{0}\right)}^{z \exp h_{0}} P(u) d u>\frac{1.2 h_{0} z^{3 / 2}}{\ln z}+O\left(\frac{z^{3 / 2}}{\ln ^{2} z}\right) .
$$

Положив $a_{1}=\max \left(L_{1} e^{2 h_{0}}, 1 / h_{0}\right)$, приходим к следуюшему утверждению. При всех $x>x_{2}$

$$
\int_{x}^{a_{1} x} P^{+}(u) d u>h_{0} x^{3 / 2}(\ln x)^{-1}
$$

Отсюда находим

$$
\int_{a_{1} x}^{a_{1}^{2} x} P^{+}(u) d u>h_{0} a_{1}^{3 / 2} x^{3 / 2}\left(\ln \left(a_{1} x\right)\right)^{-1}>\frac{\sqrt{a_{1}} x^{3 / 2}}{\ln a_{1}+\ln x}>x^{3 / 2}(\ln x)^{-1} .
$$

Тем самым утверждение 3 теоремы 14 доказано, и, следовательно, теорема 14 доказана полностью.

Из теоремы 14 легко получить следствие 11. Неравенства (19.2) и (20.2) вытекают из $(17.2)$ и непосредственно проверяемых оценок $K(1,2)>0.6, K(2)>0.85$. (Напомним, что $\omega<0.0456$. Это было отмечено в $\S 2$ перед формулировкой теоремы 13.$)$ Неравенство (21.2) является тривиальным следствием (20.2), а утверждение 2 теоремы 14 немедленно влечет за собой (22.2).

ДОКАЗАТЕЛЬСТвО тЕОРЕМЫ 12. Рассмотрим сначала самый простой случай: $\Theta>1 / 2$ является достижимым. Из (1) и леммы 1 находим

$$
P(u)=\frac{R(u)}{\ln u}+O_{\Theta}\left(\frac{u^{\Theta}}{\ln ^{2} u}\right) .
$$

Следовательно, при любом $a>1$ из (83.4) и теоремы 8 получаем

$$
\begin{aligned}
\int_{x}^{a x}|P(u)| d u & =\int_{x}^{a x} \frac{|R(u)|}{\ln u} d u+O_{\Theta}\left(\frac{x^{1+\Theta}}{\ln ^{2} x}\right) \\
& \geqslant(\ln a x)^{-1} \int_{x}^{a x}|R(u)| d u-c_{64} x^{1+\Theta}\left(\ln ^{-2} x\right) \\
& \geqslant c_{16}(\ln a x)^{-1} x^{1+\Theta}-c_{64} x^{1+\Theta} \ln ^{-2} x>c_{22} x^{1+\Theta}\left(\ln ^{-1} x\right)
\end{aligned}
$$

при $x>c_{24}$. Неравенство (16.2) доказано.

Теперь пусть $\Theta>1 / 2$ недостижимо. Обозначим

$$
B(x)=\frac{R(x)}{\ln x}, \quad \mathscr{P}(x)=B(x)+\int_{2}^{x} \frac{B(t) d t}{t \ln t} .
$$

\footnotetext{
${ }^{9}$ Если $0<q<0.1$, то $\operatorname{sh}(3 q / 2)<(7 / 4) q$.
} 
Поскольку $P(x)=\mathscr{P}(x)+O(\sqrt{x} / \ln x), x \geqslant 2$, достаточно доказать, что при любых $a>1, \varepsilon>0$ и $x>x_{3}(a, \varepsilon)$ справедливо неравенство

$$
\int_{x}^{a x}|\mathscr{P}(u)| d u>x_{n}^{1+\Theta-\varepsilon}
$$

Доказательство поведем от противного. Предположим, что сформулированное нами только что утверждение неверно. Это означает, что сушествуют $\delta>0, a>1$ и последовательность $x_{n} \rightarrow+\infty$ такие, что

$$
\int_{x_{n}}^{a x_{n}}|\mathscr{P}(u)| d u \leqslant x^{1+\Theta-\delta} .
$$

Положим

$$
\begin{gathered}
\int_{2}^{x_{n}} \frac{B(t)}{t \ln t} d t=\mathscr{D}_{n}, \quad \varphi_{n}(x)=\frac{\mathscr{D}_{n} \ln x_{n}}{\ln x}, \\
B_{n}(x)=B(x)+\varphi_{n}(x) .
\end{gathered}
$$

Легко сообразить, что имеет место равенство

$$
\mathscr{P}(u)=B_{n}(u)+\int_{x_{n}}^{u} \frac{B_{n}(t)}{t \ln t} d t, \quad u>x_{n} .
$$

Обозначим

$$
\beta_{n}(u)=\int_{x_{n}}^{u} \frac{B_{n}(t)}{t \ln t} d t, \quad K_{n}=\left\{(u, t) \in \mathbb{R}^{2} \mid x_{n} \leqslant u \leqslant a x_{n}, x_{n} \leqslant t \leqslant u\right\}
$$

и оценим сверху

$$
I_{n}=\int_{x_{n}}^{a x_{n}}\left|\beta_{n}(u)\right| d u
$$

Имеем:

$$
\begin{aligned}
I_{n} & \leqslant \int_{x_{n}}^{a x_{n}} \int_{x_{n}}^{u} \frac{\left|B_{n}(t)\right| d t}{t \ln t} d u=\iint_{K_{n}} \frac{\left|B_{n}(t)\right|}{t \ln t} d t d u \\
& =\int_{x_{n}}^{a x_{n}} \frac{\left|B_{n}(t)\right|\left(a x_{n}-t\right)}{t \ln t} d t \leqslant \frac{a-1}{\ln x_{n}} \int_{x_{n}}^{a x_{n}}\left|B_{n}(t)\right| d t .
\end{aligned}
$$

Из (86.4) и (87.4) находим, что при $n>n_{0}$

$$
\begin{aligned}
\int_{x_{n}}^{a x_{n}}|\mathscr{P}(u)| d u & \geqslant \frac{1}{2} \int_{x_{n}}^{a x_{n}}\left|B_{n}(t)\right| d t \\
& =\frac{1}{2} \int_{x_{n}}^{a x_{n}}\left|\frac{R(t)+\mathscr{D}_{n} \ln x_{n}}{\ln t}\right| d t \\
& \geqslant \frac{1}{2 \ln \left(a x_{n}\right)} \int_{x_{n}}^{a x_{n}}\left|R(t)+k_{n}\right| d t
\end{aligned}
$$


где $k_{n}=\mathscr{D}_{n} \ln x_{n}$. Очевидно, что если $f-$ произвольная функция, интегрируемая по Лебегу на множестве конечной меры $\omega \subset \mathbb{R}$, то

$$
\inf _{k \in \mathbb{R}}\|f+k\|_{L(\omega)} \geqslant \min \left(\left\|f^{+}\right\|_{L(\omega)},\left\|f^{-}\right\|_{L(\omega)}\right)
$$

Ввиду сказанного заключаем, что при $n>n_{1}>n_{0}$ справедлива оценка

$$
\int_{x_{n}}^{a x_{n}}|\mathscr{P}(u)| d u \geqslant 0.4\left(\ln x_{n}\right)^{-1} \min \left(\int_{x_{n}}^{a x_{n}} R^{+}(u) d u, \int_{x_{n}}^{a x_{n}} R^{-}(u) d u\right) .
$$

Согласно теореме 10 в случае недостижимого $\Theta$ при $x>x_{1}(\delta / 2, a)$

$$
\int_{x}^{a x} R^{+}(u) d u>x^{1+\Theta-\delta / 2}, \quad \int_{x}^{a x} R^{-}(u) d u>x^{1+\Theta-\delta / 2} .
$$

Следовательно, при $n>n_{2}>n_{1}$

$$
\int_{x_{n}}^{a x_{n}}|\mathscr{P}(u)| d u>x_{n}^{1+\Theta-\delta}
$$

а это противоречит предположению (85.4). Тем самым (84.4), а значит, и (20.2) доказано. Из (21.2), (20.2) и (18.2) при $x>c_{19}$ получаем оценку

$$
\int_{x}^{2 x}|P(u)| d u>x^{3 / 2}(\ln x)^{-1}
$$

вне зависимости от значения $\Theta$. Если проследить доказательство теоремы 14 и следствия 11 , то видно, что в случае $\Theta=1 / 2$ постоянная $c_{19}$ является эффективной. Теорема 12 полностью доказана.

\section{§. Доказательства теорем 15-19}

ДоКАЗАТЕЛЬСТВо ТЕОРЕМЫ 18. Согласно определению $R, R_{0}, \mathscr{F},(37.2)$ и $(38.3)$ при $x \geqslant 2$ имеем

$$
\begin{aligned}
\int_{x}^{x+v x} R^{2}(u) d u & =\int_{x}^{x+v x} R_{0}^{2}(u) d u=\int_{x}^{x+v x}(\mathscr{F}(u)+O(1))^{2} d u \\
& \leqslant 2 \int_{x}^{x+v x} \mathscr{F}^{2}(u) d u+O\left(\int_{x}^{x+v x} d u\right) \\
& \leqslant 2 \int_{x}^{x+v x} \mathscr{F}^{2}(u) d u+c_{65} v x
\end{aligned}
$$


Интегрируя почленно возведенньй в квадрат ряд $(37.2)$ для $\mathscr{F}(u),{ }^{10}$ получаем ${ }^{11}$

$$
\begin{aligned}
\frac{1}{x^{2}} \int_{x}^{x+v x} \mathscr{F}^{2}(u) d u & =\frac{1}{x^{2}} \int_{x}^{x+v x} \sum_{\rho_{1}} \sum_{\rho_{2}} \frac{u^{1+i\left(\gamma_{1}+\gamma_{2}\right)}}{\rho_{1} \rho_{2}} d u \\
& =\frac{1}{x^{2}} \sum_{\rho_{1}} \sum_{\rho_{2}} \frac{(x+v x)^{2+i\left(\gamma_{1}+\gamma_{2}\right)}-x^{2+i\left(\gamma_{1}+\gamma_{2}\right)}}{\rho_{1} \rho_{2}\left(2+i\left(\gamma_{1}+\gamma_{2}\right)\right)} \\
& \leqslant \sum_{\rho_{1}} \sum_{\rho_{2}} \frac{\left|(1+v)^{2+i\left(\gamma_{1}+\gamma_{2}\right)}-1\right|}{\left|\rho_{1} \rho_{2}\right| \sqrt{4+\left(\gamma_{1}+\gamma_{2}\right)^{2}}}:=\sigma(v) .
\end{aligned}
$$

В свете введенного обозначения осталось доказать, что при $v \in(0,1 / 3)$

$$
\sigma(v)=O\left(v \ln ^{4} v\right) .
$$

Положим $T=[1 / v]$ и разобьем двойной ряд $\sigma(v)$ на “начало” и “остаток":

$$
\begin{aligned}
\sigma(v) & =\sigma_{1}(v)+\sigma_{2}(v), \\
\sigma_{1}(v) & =\sum_{\left|\gamma_{1}\right| \leqslant T} \sum_{\left|\gamma_{2}\right| \leqslant T} \frac{\left|(1+v)^{2+i\left(\gamma_{1}+\gamma_{2}\right)}-1\right|}{\left|\rho_{1} \rho_{2}\right| \sqrt{4+\left(\gamma_{1}+\gamma_{2}\right)^{2}}}, \\
\sigma_{2}(v) & =\sigma(v)-\sigma_{1}(v) .
\end{aligned}
$$

Начало ряда оценим, пользуясь неравенствами $\left(s=1+i\left(\gamma_{1}+\gamma_{2}\right), 0<v<1\right)$

$$
\left|\frac{(1+v)^{s+1}-1}{s+1}\right|=\left|\int_{0}^{v}\left(1+t^{s}\right) d t\right| \leqslant v(1+v)^{\operatorname{Re} s}=v(1+v)<2 v .
$$

Получаем

$$
\begin{aligned}
\sigma_{1}(v) & \leqslant 2 v \sum_{\left|\gamma_{1}\right| \leqslant T} \sum_{\left|\gamma_{2}\right| \leqslant T} \frac{1}{\left|\rho_{1} \rho_{2}\right|} \\
& =2 v\left(\sum_{|\gamma| \leqslant T}|\rho|^{-1}\right)^{-2}=O\left(v \ln ^{4} T\right)=O\left(v \ln ^{4} v\right) .
\end{aligned}
$$

Теперь оценим остаток ряда. Воспользуемся очевидным представлением

$$
\sigma_{2}(v)=\sum_{N=T}^{\infty} A(N)
$$

где

$$
A(N)=\sum_{\left(\gamma_{1}, \gamma_{2}\right)} \sum_{\in \Pi(N)} \frac{\left|(1+v)^{2+i\left(\gamma_{1}+\gamma_{2}\right)}-1\right|}{\left|\rho_{1} \rho_{2}\right| \sqrt{4+\left(\gamma_{1}+\gamma_{2}\right)^{2}}}
$$

\footnotetext{
10 Законность этой операции была обоснована в доказательстве теоремы 1.

11 десь мы снова, как и в доказательстве теоремы 1 , нетривиальные нули $\zeta(s)$ не нумеруем, а через $\rho_{1}$ и $\rho_{2}$ обозначаем соответственно первую и вторую компоненты декартова квадрата множества $\{\rho\}$.
} 
$\Pi(N)=K_{N+1} \backslash K_{N}$, а $K_{r}-$ квадрат в $\mathbb{R}^{2}$ следуюшего вида: $K_{r}=[-r, r] \times[-r, r]$. $\Pi(N)$, в свою очередь, разобьем на квадратики со сторонами длины 1 , параллельными координатным осям. В суммах $A(N)$ тривиально оценим числитель:

$$
\left|(1+v)^{2+i\left(\gamma_{1}+\gamma_{2}\right)}-1\right| \leqslant(1+v)^{2}+1<3 .
$$

Из соображений симметрии ясно, что суммы от $\left|\rho_{1} \rho_{2}\right|^{-1}\left(4+\left(\gamma_{1}+\gamma_{2}\right)^{2}\right)^{-1 / 2}$ по любому из четырех рядов квадратиков (верхнему и нижнему горизонтальным, левому и правому вертикальным) совпадают между собой. Поэтому

$$
A(N)<12 B(N)
$$

где

$$
\begin{gathered}
B(N)=\sum_{m=-1-N}^{N} \sum_{\left(\gamma_{1}, \gamma_{2}\right) \in q_{m}} \frac{1}{\left|\rho_{1} \rho_{2}\right| \sqrt{4+\left(\gamma_{1}+\gamma_{2}\right)^{2}}}, \\
q_{m}=\left\{\left(y_{1}, y_{2}\right) \in \mathbb{R}^{2} \mid N<y_{1}<N+1, m \leqslant y_{2}<m+1\right\} .
\end{gathered}
$$

Количество элементов последовательности $\left(\gamma_{1}, \gamma_{2}\right)$ в квадратике $q_{m}$ составляет $O(\ln N \cdot \ln m)$, если $|m| \geqslant 14$, и равно нулю, если $|m|<14$. К тому же $\left|\rho_{1}\right|>N$. Ввиду сказанного приходим к оценкам

$$
\begin{aligned}
B(N) & <\frac{\ln N}{N} \sum_{14 \leqslant|m| \leqslant N+1} \frac{\ln m}{m \cdot|\max (2, N+m)|} \\
& <\frac{2 \ln N}{N}\left(\frac{\ln N}{N}+\sum_{m=14}^{N-1} \frac{\ln m}{m(N-m)}\right)<c_{66} \frac{\ln ^{3} N}{N^{2}} .
\end{aligned}
$$

Из (5.5)-(7.5) находим

$$
\sigma_{2}(v) \leqslant c_{67} T^{-1} \ln ^{3} T
$$

Из (1.5)-(4.5) и (8.5) получаем (32.2). Оценка (33.2) сразу же получается из (32.2) с помошью неравенства Коши-Буняковского:

$$
\begin{aligned}
\left|R_{1}(x, H(x))\right| & \leqslant(H(x))^{-1} \int_{x}^{x+H(x)}|R(u)| d u \\
& \leqslant(H(x))^{-1}\left(\int_{x}^{x+H(x)} R^{2}(u) d u\right)^{1 / 2} \cdot\left(\int_{x}^{x+H(x)} d u\right)^{1 / 2} \\
& =O(H(x))^{-1} \sqrt{x H(x) \ln ^{4}\left(\frac{x}{H(x)}\right)} \sqrt{H(x)} \\
& =O\left(\sqrt{x} \ln ^{2}\left(\frac{x}{H(x)}\right)\right) .
\end{aligned}
$$


ДоКАЗАТЕЛЬСТво тЕОРемы 19 поведем от противного. Допустим, что существует некоторая функция $H(x), 1 \leqslant H(x)=o(x)(x \rightarrow+\infty)$, такая, что выражение

$$
E(x)=\frac{1}{\sqrt{x} H(x)} \int_{x}^{x+H(x)} R(u) d u
$$

ограничено либо сверху, либо снизу. Мы разберем случай ограниченности $E(x)$ сверху. Неограниченность снизу $E(x)$ доказывается совершенно аналогично.

Итак, предположим, что существует постоянная $K \geqslant 1$ такая, что при любых $x>2$

$$
\int_{x}^{x+H(x)} R(u) d u<K \sqrt{x} H(x) .
$$

Положим

$$
h=\min \left(c_{53}, \exp \left(-20 K-10 c_{52}\right)\right),
$$

где $c_{52}, c_{53}$ - постоянные лемм 8 и 9 соответственно. По лемме 9 сушествует последовательность $t_{n} \rightarrow+\infty$ такая, что выполнены неравенства

$$
S\left(t_{n}, h\right)<0.1 \ln h \leqslant-2 K-c_{52} .
$$

Обозначим $z_{n}=\exp \left(t_{n}\right)$. Лемма 8 вместе с (10.5) дает

$$
\frac{1}{2 h z_{n}^{3 / 2}} \int_{z_{n} e^{-h}}^{z_{n} e^{h}} R(u) d u>4 K \quad \forall n \in \mathbb{N} .
$$

Полагая $z_{n} e^{-h}=x_{n}, \alpha=e^{2 h}-1$ (ясно, что $\left.2 h>3 \alpha / 4\right)$, получаем

$$
\int_{x_{n}}^{x_{n}+\alpha x_{n}} R(u) d u>3 K \alpha x_{n}^{3 / 2}
$$

Покажем, что оценки (11.5) и (9.5) при больших $x_{n}$ несовместны. Получившееся противоречие и будет означать неограниченность сверху функции $x^{-1 / 2} R_{1}(x, H(x))$.

Зададим рекуррентно последовательности чисел $x_{k, n}$. Положим

$$
x_{0, n}=x_{n}, \quad x_{k+1, n}=x_{k, n}+H\left(x_{k, n}\right), \quad k=0,1,2, \ldots
$$

Пусть $N$ - такой номер, что

$$
x_{N, n} \leqslant x_{n}+\alpha x_{n}<x_{N+1, n} .
$$

Он обязательно сушествует, так как $H(x) \geqslant 1$, и, следовательно, $\lim _{k \rightarrow \infty} x_{k, n}=+\infty$. Из (9.5) находим

$$
\int_{x_{k, n}}^{x_{k+1, n}} R(u) d u=\int_{x_{k, n}}^{x_{k, n}+H\left(x_{k, n}\right)} R(u) d u<K \sqrt{x_{k, n}} H\left(x_{k, n}\right) .
$$


Но, так как при $k \leqslant N$ имеем $x_{k, n} \leqslant x_{n}+\alpha x_{n}<2 x_{n}$, то $\sqrt{x_{k, n}} \leqslant \sqrt{2 x_{n}}, 0 \leqslant k \leqslant N-1$, и, складывая неравенства (13.5), приходим к соотношениям

$$
\begin{aligned}
\int_{x_{n}}^{x_{N, n}} R(u) d u & <\sqrt{2 x_{n}} K \sum_{k=0}^{N-1} H\left(x_{k, n}\right) \\
& =\sqrt{2 x_{n}} K \sum_{k=0}^{N-1}\left(x_{k+1, n}-x_{k, n}\right) \\
& =\sqrt{2 x_{n}} K\left(x_{N, n}-x_{0, n}\right) \leqslant \alpha x_{n} \sqrt{2 x_{n}} K .
\end{aligned}
$$

Обозначим $v_{n}=\left(x_{n}+\alpha x_{n}-x_{N, n}\right) / x_{N, n}$. Из (13.5) следует, что $v_{n}<H\left(x_{N, n}\right) / x_{N, n}$. Применяя теорему 18, находим

$$
\begin{aligned}
\left|\int_{x_{N, n}}^{x_{n}+\alpha x_{n}} R(u) d u\right| & =\left|\int_{x_{N, n}}^{x_{N, n}+v_{n} x_{N, n}} R(u) d u\right| \\
& =O\left(x_{n}^{3 / 2} v_{n} \ln ^{2} v_{n}\right)=o\left(x_{n}^{3 / 2}\right) \quad(n \rightarrow+\infty),
\end{aligned}
$$

так как $\lim _{n \rightarrow \infty} v_{n}=0$.

Вследствие (14.5) и (15.5) при достаточно больших $n$ получаем неравенство

$$
\int_{x_{N, n}}^{x_{n}+\alpha x_{n}} R(u) d u<2 K \alpha x_{n}^{3 / 2}
$$

которое противоречит (11.5). Как отмечалось выше, это доказывает неограниченность сверху $x^{-1 / 2} R_{1}(x, H(x))$. Неограниченность снизу этой функции доказьвается совершенно аналогично. Нужно лишь вместо оценки сверху $S\left(y_{2}, h\right)$ воспользоваться оценкой снизу $S\left(y_{1}, h\right)$ из леммы 9 . Теорема 19 доказана.

ДОКАЗАТЕЛЬСТВО ТЕОРЕмЫ 17 . Сначала с помощью леммы 8 перепишем утверждение теоремы 17 в более удобном для доказательства виде. Положим

$$
z=\sqrt{x(x+H(x))}, \quad h_{0}=h_{0}(x)=\frac{1}{2} \ln \left(1+\frac{H(x)}{x}\right) .
$$

Тогда $z \exp \left(-h_{0}\right)=x, z \exp \left(h_{0}\right)=x+H(x)$. По лемме 8

$$
\begin{aligned}
& \frac{1}{2 h_{0} z^{3 / 2}} \int_{z \exp \left(-h_{0}\right)}^{z \exp \left(h_{0}\right)} R(u) d u \\
& \quad=\frac{H(x) / x}{\ln (1+H(x) / x)} \cdot x^{-1 / 2}(1+H(x) / x)^{-3 / 4} \frac{1}{H(x)} \int_{x}^{x+H(x)} R(u) d u \\
& \quad=\frac{(1+o(1))}{\sqrt{x}} R_{1}(x, H(x)) .
\end{aligned}
$$

Отсюда и из (67.4) находим (определение функции $S$ см. в (66.4))

$$
R_{1}(x, H(x))=\sqrt{x}(1+o(1))\left(S\left(\ln z, h_{0}\right)+O(1)\right) .
$$


Поэтому требуется доказать, что

$$
S(y, a(y))=\Omega_{ \pm}(\ln (a(y)))
$$

где

$$
y=\ln z, \quad a(y)=\frac{1}{2} \ln \left(1+\frac{H(x)}{x}\right) \sim \frac{1}{(2 V(x))} \quad(x \rightarrow+\infty) .
$$

Из условий, наложенных на функцию $H$, получим условия на функцию $a$ :

$$
\begin{gathered}
a(y) \searrow 0 \quad(y+\infty), \quad a \in C^{1}\left(y\left(x_{0}\right),+\infty\right), \\
\frac{d a}{d y}=\frac{d a}{d x} \frac{d x}{d y}=\frac{d a}{d x} \frac{d x}{d z} \frac{d z}{d y}=O\left(\frac{1}{x \ln x}\right)(1+o(1)) e^{y} \\
=O(1 / \ln x)=O(1 / y) \quad(y \rightarrow+\infty) \\
\ln \frac{1}{a\left(y^{2}\right)}=\ln \frac{1}{a\left(z^{\ln z}\right)}=\ln V\left(z^{\ln z}\right) \\
=O(\ln V(z))=O(\ln (1 / a(y))) .
\end{gathered}
$$

Итак, осталось доказать $\Omega_{ \pm}$-оценку $(16.5)$ для любой функции $a(y)$, удовлетворяющей условиям (17.5)-(19.5). Положим

$$
T(h)=h^{-1} \ln (1 / h), \quad h(Y)=\sqrt{a(Y)} .
$$

При $Y>x_{4}>x_{3}$ ввиду (17.5) имеем $h(Y)<c_{52}$. Поэтому согласно неравенствам, доказанным в лемме 9 , для любых $Y>x_{4}$ и $h=h(Y)$ найдутся числа $y_{1}, y_{2} \in$ $\left[Y, Y \exp \left(h^{-1} \ln ^{2.5}(1 / h)\right)\right]$, для которых

$$
\begin{aligned}
& S_{1}\left(y_{1}, h\right)>0.1 \ln (1 / h), \\
& S_{1}\left(y_{2}, h\right)<0.1 \ln h .
\end{aligned}
$$

Из (20.5) несложно вывести, что при $Y>x_{5}>x_{4}$

$$
\begin{gathered}
S_{2}\left(y_{1}, a(y), h\right)>\frac{1}{30} \ln \left(\frac{1}{a(Y)}\right), \\
S_{2}\left(y_{2}, a(y), h\right)<\frac{1}{30} \ln (a(Y)),
\end{gathered}
$$

где

$$
S_{2}\left(y_{1}, a(y), h\right)=\sum_{0<\gamma<T(h)} \frac{\sin \gamma y}{\gamma} \frac{\sin \gamma h}{\gamma h} \frac{\sin \gamma a(y)}{\gamma a(y)} .
$$

(Напомним, что здесь $h=\sqrt{a(Y)}$.) Действительно, при $|z|<1$ имеем $1-(\sin z) / z=$ $O\left(z^{2}\right)$, а если $\gamma \in[0, T(h(Y))]$ и $y \geqslant Y$, то

$$
0<\gamma a(y)<T(h(Y)) a(Y)=O(\sqrt{a(Y)} \ln (1 / a(Y))) .
$$


Поэтому

$$
\begin{aligned}
\left|S_{1}(y, h)-S_{2}(y, a(y), h)\right| & =O\left(a(Y) \ln ^{2}(1 / a(Y)) \sum_{0<\gamma<T(h)}\left|\frac{\sin \gamma y}{\gamma} \frac{\sin \gamma h}{\gamma h}\right|\right) \\
& =O\left(a(Y) \ln ^{2}(a(Y)) \sum_{0<\gamma<T(h)} \gamma^{-1}\right) \\
& =O\left(a(Y) \ln ^{4}(a(Y))\right)=o(1) \quad(y \geqslant Y \rightarrow+\infty) .
\end{aligned}
$$

Тем самым из (20.5) и (22.5) следуют неравенства (21.5) при $Y>x_{5}$.

Теперь покажем, что $S_{2}(y, a(y), h)$ "мало отличается" от среднего для функции $S(t, a(t))$ на отрезке $[y-h, y+h]$. Имеем:

$$
\begin{aligned}
\frac{1}{2 h} \int_{y-h}^{y+h} S(t, a(t)) d t= & \frac{1}{2 h} \sum_{\gamma>0} \int_{y-h}^{y+h} \frac{\sin \gamma t}{\gamma} \frac{\sin \gamma a(t)}{\gamma a(t)} d t \\
= & \frac{1}{2 h} \sum_{\gamma>0} \frac{\sin \gamma a(y)}{\gamma a(y)} \int_{y-h}^{y+h} \frac{\sin \gamma t}{\gamma} d t \\
& +\frac{1}{2 h} \sum_{\gamma>0} \int_{y-h}^{y+h} \frac{\sin \gamma t}{\gamma}\left(\frac{\sin \gamma a(t)}{\gamma a(t)}-\frac{\sin \gamma a(y)}{\gamma a(y)}\right) d t \\
= & S_{3}(y, a(y), h)+O\left(S_{4}(y, a(y), h)\right)
\end{aligned}
$$

где

$$
\begin{aligned}
& S_{3}(y, a(y), h)=\sum_{\gamma>0} \frac{\sin \gamma y}{\gamma} \frac{\sin \gamma h}{\gamma h} \frac{\sin \gamma a(y)}{\gamma a(y)} \\
& S_{4}(y, a(y), h)=h^{-1} \sum_{\gamma>0} \gamma^{-1} \int_{y-h}^{y+h}\left|\frac{\sin \gamma a(t)}{\gamma a(t)}-\frac{\sin \gamma a(y)}{\gamma a(y)}\right| d t .
\end{aligned}
$$

Рассуждая так же, как и в доказательстве леммы 9, получаем соотношение

$$
S_{2}(y, a(y), h)-S_{3}(y, a(y), h)=O(1)
$$

Оценим сумму $S_{4}$. С этой целью разобьем ее на две части:

$$
S_{4}=S_{5}+S_{6}, \quad \text { где } S_{5}=\sum_{\gamma \leqslant a^{-2}(y)}, S_{6}=\sum_{\gamma>a^{-2}(y)} .
$$

В сумме $S_{6}$ оценим интеграл тривиально:

$$
\begin{aligned}
\frac{1}{2 h} \int_{y-h}^{y+h}\left|\frac{\sin \gamma t}{\gamma}-\frac{\sin \gamma a(y)}{\gamma a(y)}\right| d t & \leqslant \max _{t \in[y-h, y+h]}\left|\frac{\sin \gamma a(t)}{\gamma a(t)}-\frac{\sin \gamma a(y)}{\gamma a(y)}\right| \\
& \leqslant \frac{2}{\gamma a(y+h)}<\frac{2}{\gamma a(y+1)} .
\end{aligned}
$$


Поэтому $^{12}$

$$
\begin{aligned}
S_{6} & \leqslant \frac{4}{a(y+1)} \sum_{\gamma>a^{-2}(y)} \gamma^{-2} \\
& =O\left(\frac{a^{2}(y) \ln (1 / a(y))}{a(y+1)}\right)=o\left(\frac{a(y)}{a(y+1)}\right)=o(1) \quad(y \rightarrow+\infty) .
\end{aligned}
$$

В сумме $S_{5}$ для $f(t)=f_{\gamma}(t)=\frac{\sin \gamma a(t)}{\gamma a(t)}$ оценим интеграл следуюшим образом:

$$
\begin{aligned}
h^{-1} \int_{y-h}^{y+h}|f(t)-f(y)| d t & \leqslant h^{-1} \int_{y-h}^{y+h}\left|(t-y) f^{\prime}(\xi(t, y))\right| d t \\
& \leqslant \max _{u \in[y-h, y+h]}\left|f^{\prime}(u)\right| \cdot h^{-1} \int_{y-h}^{y+h}|t-y| d t \\
& =h \cdot \max _{u \in[y-h, y+h]}\left|f^{\prime}(u)\right| .
\end{aligned}
$$

Поэтому

$$
S_{5} \leqslant h \sum_{0<\gamma<a^{-2}(y)} \gamma^{-1} \max _{u \in[y-h, y+h]} \frac{d}{d t}\left(\frac{\sin \gamma a(t)}{\gamma a(t)}\right) .
$$

Так как

$$
\frac{d}{d \alpha}\left(\frac{\sin \alpha}{\alpha}\right) \leqslant \min (\alpha, 2 / \alpha) \quad \forall \alpha \in \mathbb{R}
$$

и, как уже отмечалось, справедливы соотношения

$$
a(u) \sim a(y), \quad u \in[y-h, y+h], \quad a^{\prime}(u)=O(1 / u),
$$

то из (26.5) с учетом (18.5) и (36.2) получаем оценки

$$
\begin{aligned}
S_{5} & =O\left(h y^{-1} \sum_{0<\gamma<a^{-2}(y)} \min (\gamma a(y), 1 /(\gamma a(y)))\right) \\
& =O\left(h y^{-1} \frac{\ln (1 / a(y))}{a(y)}\right)=o\left(y^{-1} \ln y \ln \ln y\right)=o(1) \quad(y \rightarrow+\infty) .
\end{aligned}
$$

Из (23.5)-(25.5) и (27.5) находим

$$
S_{2}(y, a(y), h)=\frac{1}{2 h} \int_{y-h}^{y+h} S(t, a(t)) d t+O(1), \quad y \geqslant Y .
$$

Соотношения (22.5) и (28.5) позволяют заключить, что

$$
\begin{gathered}
S(t, a(t))=\Omega_{ \pm} \ln (a(Y)), \\
t \in\left[Y-h(Y), h(Y)+Y \exp \left(h^{-1}(Y)\right) \ln ^{2.5}\left(h^{-1}(Y)\right)\right] .
\end{gathered}
$$

Но, исходя из выбора $h(Y)$ и неравенства $a(Y) \geqslant 1 / 4 \ln Y$, нетрудно проверить, что правьй конец промежутка (29.5) не превосходит $Y^{2}$, а ввиду (19.5) и монотонности $a(t)$ при $t \in\left[Y, Y^{2}\right]$ имеем $\ln (a(t)) \asymp \ln (a(Y))$. Поэтому

$$
S_{2}(t, a(t))=\Omega_{ \pm} \ln (a(t))
$$

а это доказьвает теорему 17.

\footnotetext{
${ }^{12}$ Из (17.5)-(18.5) легко следует, что $\lim _{y \rightarrow+\infty}(a(y) / a(y+1))=1$.
} 
ЛЕмма 10. Обозначим $A(x)=\int_{x}^{2 x} R(u) d u$. Тогда

$$
\begin{aligned}
& A(x)=\Omega_{ \pm}\left(x^{1+\Theta-\varepsilon}\right) \quad \forall \varepsilon>0 \\
& A(x)=\Omega_{ \pm}\left(x^{1+\Theta}\right), \quad \text { если } \Theta \text { достижимо. }
\end{aligned}
$$

ДокАЗАТЕЛЬСТво. Хорошо известно [16, с. 50] интегральное представление

$$
-\frac{\zeta^{\prime}(s)}{s \zeta(s)}-\frac{1}{s-1}=\int_{1}^{+\infty} R(u) u^{-s-1} d u, \quad \operatorname{Re} s>\Theta .
$$

Произведя в правой части (30.5) интегрирование по частям, приходим к соотношению $\left(R_{1}(u)=\int_{1}^{u} R(t) d t\right)$

$$
\int_{1}^{+\infty} R_{1}(u) u^{-s-2} d u=-\frac{1}{s+1}\left(\frac{1}{s-1}+\frac{\zeta^{\prime}(s)}{s \zeta(s)}\right), \quad \operatorname{Re} s>\Theta .
$$

Поскольку $A(x)=R_{1}(2 x)-R(x)$, из (32.2) получаем аналитическое выражение для преобразования Меллина функции $A(u)$ :

$$
\begin{aligned}
f(s) & =\int_{1}^{+\infty} A(u) u^{-s-2} d u \\
& =\int_{1 / 2}^{+\infty} R_{1}(2 u) u^{-s-2} d u-\int_{1}^{+\infty} R_{1}(u) u^{-s-2} d u-\int_{1 / 2}^{1} R_{1}(2 u) u^{-s-2} d u \\
& =-\frac{2^{s+1}-1}{s+1}\left(\frac{1}{s-1}+\frac{\zeta^{\prime}(s)}{s \zeta(s)}\right)+\text { целая функция. }
\end{aligned}
$$

Таким образом, $f(s)$ - преобразование Меллина функции $A(u)$ - регулярно на луче $(0,+\infty)$ и все особые точки $f(s)$ в полуплоскости $\operatorname{Re} s>0$ - изолированные, совпадают с нулями $\zeta(s)$ и являются полюсами. Воспользуемся теперь одной известной тауберовой теоремой.

Пусть $g(x)$ - всюду конечная измеримая действительнозначная функция на $[1,+\infty)$, $g(x)=O\left(x^{\lambda}\right)(x \geqslant 1)$, где $\lambda$ - некоторая постоянная. Пусть затем преобразование Меллина функции $g$

$$
G(s)=\int_{1}^{+\infty} g(x) x^{-s-1} d x
$$

имеет в $\mathbb{C}$ полюс в точке $s=\beta+i \gamma, \gamma \neq 0$, и регулярно на луче $[\beta,+\infty)$. Тогда

$$
g(x)=\Omega_{ \pm}\left(x^{\beta}\right) \quad(x \rightarrow+\infty) .
$$

Из этой теоремы и представления (32.5) без труда получаем утверждение леммы 10. 
ДОКАЗАТЕЛЬСТВО ТЕОРЕМЫ 16.

I. $\Theta=1 / 2$. Идея доказательства очень проста. Предположив, что либо

$$
\limsup _{x \rightarrow+\infty} \frac{R_{1}(x, H(x))}{\sqrt{x} \ln \ln \ln x} \leqslant 0,
$$

либо

$$
\liminf _{x \rightarrow+\infty} \frac{R_{1}(x, H(x))}{\sqrt{x} \ln \ln \ln x} \geqslant 0,
$$

мы для функции $H_{0}(x)=x / \sqrt{\ln \ln x}$ из (33.5) получим

$$
\limsup _{x \rightarrow+\infty} \frac{R_{1}\left(x, H_{0}(x)\right)}{\sqrt{x} \ln \ln \ln x} \leqslant 0,
$$

а из (34.5) находим, что

$$
\liminf _{x \rightarrow+\infty} \frac{R_{1}\left(x, H_{0}(x)\right)}{\sqrt{x} \ln \ln \ln x} \geqslant 0
$$

Но, применив к $R_{1}\left(x, H_{0}(x)\right)$ теорему 17 (функция $H_{0}$ удовлетворяет всем условиям этой теоремы), убеждаемся в том, что оба соотношения (35.5) и (36.5) неверны. Зна-

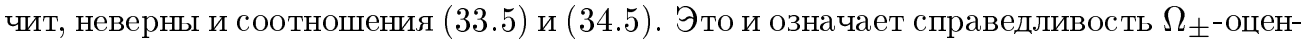
ки (29.2).

Установим справедливость импликации $(33.5) \Rightarrow(35.5)$. С каждым $x>x_{0}$ свяжем последовательность чисел $a_{n}(x)$ :

$$
a_{0}(x)=x, \quad a_{n}(x)=a_{n-1}(x)+H\left(a_{n-1}(x)\right), \quad n \in \mathbb{N} .
$$

Пусть $N=N(x)$ - номер, для которого выполнено неравенство

$$
a_{N}(x) \leqslant x+H_{0}(x)<a_{N+1}(x) .
$$

Ввиду (33.5) найдется монотонно стремящаяся к нулю положительная функция $\varepsilon(z)$ такая, что

$$
\int_{z}^{z+H(z)} R(u) d u<\varepsilon(z) \sqrt{z} H(z) \ln \ln \ln z .
$$

Применяя $(37.5)$ к $z=a_{k}(x), k=0,1, \ldots, N-1$, находим

$$
\begin{aligned}
\int_{a_{k}(x)}^{a_{k+1}(x)} R(u) d u & <\varepsilon\left(a_{k}(x)\right) \sqrt{a_{k}(x)} H\left(a_{k}(x)\right) \ln \ln \ln \left(a_{k}(x)\right) \\
& =\left(a_{k+1}(x)-a_{k}(x)\right) O(\varepsilon(x) \sqrt{x} \ln \ln \ln x) .
\end{aligned}
$$

Складьвая эти неравенства, получим

$$
\int_{x}^{a_{N}(x)} R(u) d u=o\left(\sqrt{x} H_{0}(x) \ln \ln \ln x\right) \quad(x \rightarrow+\infty) .
$$


Осталось оценить интеграл $R(u)$ по "короткому" отрезку $\left[a_{N}(x), x+H_{0}(x)\right]$. Его длина $l(x)$ меньше, чем $H\left(a_{N}(x)\right) \leqslant H(2 x)$. По теореме 18

$$
\int_{a_{N}(x)}^{x+H_{0}(x)}|R(u)| d u=O\left(x^{3 / 2} \varphi(x / l(x))\right),
$$

где $\varphi(t)=t^{-1} \ln ^{2} t$. Так как $\varphi(t)$ убьвает при $t \geqslant e^{2}$, то вместо $x / l(x)$ при больших $x$ в (39.5) можно поставить оценку снизу этой величины: $x / l(x) \geqslant x / H(2 x) \geqslant$ $c_{58} \ln \ln (2 x)$. Поэтому

$$
\begin{aligned}
\int_{a_{N}(x)}^{x+H_{0}(x)}|R(u)| d u & =O\left(x^{3 / 2}(\ln \ln (2 x))^{-1}(\ln \ln \ln (2 x))^{2}\right) \\
& =o\left(\sqrt{x} H_{0}(x) \ln \ln \ln x\right) .
\end{aligned}
$$

Из (38.5) и (40.5) получаем (35.5). Импликация (34.5) $\Rightarrow(36.5)$ доказывается совершенно аналогично.

II. $\Theta>1 / 2$ достижимо. Как и вьшше, положим

$$
a_{0}(x)=x, \quad a_{n}(x)=a_{n-1}(x)+H\left(a_{n-1}(x)\right), \quad n \in \mathbb{N} .
$$

Но здесь через $N=N(x)$ обозначим номер, для которого вьполнено неравенство $a_{N}(x) \leqslant 2 x<a_{N+1}(x)$. Из теоремы 2 находим

$$
\begin{aligned}
\int_{a_{N}(x)}^{2 x}|R(u)| d u & =O\left(x^{\Theta}\left(2 x-a_{N}(x)\right)\right)=O\left(x^{\Theta} H\left(a_{N}(x)\right)\right) \\
& =o\left(x^{\Theta} a_{N}(x)\right)=o\left(x^{1+\Theta}\right) \quad(x \rightarrow+\infty) .
\end{aligned}
$$

Предположив, что $\lim \sup _{x \rightarrow+\infty} x^{-\Theta} R_{1}(x, H(x)) \leqslant 0$, получим, что существует монотонно стремящаяся к нулю положительная функция $\varepsilon(x)$, для которой справедливо неравенство

$$
\int_{x}^{x+H(x)} R(u) d u \leqslant \varepsilon(x) x^{\Theta} H(x), \quad x>x_{0} .
$$

Поэтому

$$
\begin{aligned}
\int_{a_{k}(x)}^{a_{k+1}(x)} R(u) d u & \leqslant\left(a_{k+1}(x)-a_{k}(x)\right) \varepsilon(x)\left(a_{k}(x)\right)^{\Theta} \\
& \leqslant\left(a_{k+1}(x)-a_{k}(x)\right) \varepsilon(x)(2 x)^{\Theta} .
\end{aligned}
$$

Складьвая неравенства (43.5), находим

$$
\begin{aligned}
\int_{x}^{a_{N}(x)} R(u) d u & \leqslant\left(a_{N}(x)-a_{0}(x)\right) \varepsilon(x)(2 x)^{\Theta} \\
& \leqslant 2 x^{1+\Theta} \varepsilon(x)=o\left(x^{1+\Theta}\right) \quad(x \rightarrow+\infty) .
\end{aligned}
$$


Из (42.5) и (44.5) получаем импликацию

$$
\limsup _{x \rightarrow+\infty} x^{-\Theta} R_{1}(x, H(x)) \leqslant 0 \Longrightarrow \limsup _{x \rightarrow+\infty} x^{-1-\Theta} A(x) \leqslant 0 .
$$

Аналогично доказывается, что

$$
\liminf _{x \rightarrow+\infty} x^{-\Theta} R_{1}(x, H(x)) \geqslant 0 \Longrightarrow \liminf _{x \rightarrow+\infty} x^{-1-\Theta} A(x) \geqslant 0 .
$$

Но согласно лемме 10 правые части этих импликаций неверны. Следовательно, неверны посылки. Это и означает, что $R_{1}(x, H(x))=\Omega_{ \pm}\left(x^{\Theta}\right)$. Теорема 16 полностью доказана.

Из теоремы 16 заключаем, что теорему 15 осталось доказать только в случае, когда $\Theta$ недостижимо. Снова, так же как и в (41.5), определим $a_{k}(x), 1 \leqslant k \leqslant N+1$. Предположив, что

$$
\int_{x}^{x+H(x)} R(u) d u \leqslant \varepsilon(x) \sqrt{x} H(x) \ln \ln \ln x \quad\left(x>x_{0}\right),
$$

где $\lim _{x \rightarrow+\infty} \varepsilon(x)=0, \varepsilon(x)>0$ (и, следовательно, можно без ограничения общности считать, что $\varepsilon(x) \downarrow 0)$, получаем тем же техническим приемом, что и ранее,

$$
\int_{x}^{a_{N}(x)} R(u) d u=o\left(x^{3 / 2} \ln \ln \ln x\right) \quad(x \rightarrow+\infty) .
$$

Пользуясь абсолютной оценкой $R(u)$ из теоремы 2 , находим

$$
\int_{a_{N}(x)}^{2 x}|R(u)| d u=o\left(x^{\Theta} H\left(a_{N}(x)\right)\right)=o\left(x^{1+\Theta-\delta}\right) .
$$

Возьмем теперь $\varepsilon=\min (\delta / 2, \Theta / 2-1 / 4)$. Ясно, что из (45.5) и (46.5) следуют импликации

$$
\begin{aligned}
& \limsup _{x \rightarrow+\infty} x^{-1 / 2-\varepsilon} R_{1}(x, H(x)) \leqslant 0 \Longrightarrow \limsup _{x \rightarrow+\infty} x^{\varepsilon-1-\Theta} A(x) \leqslant 0, \\
& \liminf _{x \rightarrow+\infty} x^{-1 / 2-\varepsilon} R_{1}(x, H(x)) \geqslant 0 \Longrightarrow \liminf _{x \rightarrow+\infty} x^{\varepsilon-1-\Theta} A(x) \geqslant 0 .
\end{aligned}
$$

Согласно лемме 10 правые части (47.5) и (48.5) неверны и, следовательно, неверны посылки этих импликаций. Поэтому

$$
R_{1}(x, H(x))=\Omega_{ \pm}\left(x^{1 / 2+\varepsilon}\right)=\Omega_{ \pm}(\sqrt{x} \ln \ln \ln x) .
$$

А. Ю. Попов, писавший окончательньй вариант этой работы после смерти профессора С.Б. Стечкина, приносит благодарность доктору физико-математических наук С. В. Конягину за прочтение рукописи и ценные замечания, которые помогли значительно улучшить изложение. 


\section{СПИСОК ЛИТЕРАТУРЫ}

[1] Hadamar J. Sur la distribution des zeros de la fonction $\zeta(s)$ et des consequences arithmetiques // Bull. Soc. Math. France. 1896. V. 24. P. 199-220.

[2] de la Vallée Poussin Ch. J. Recherches analytiques sur la théorie des nombres. Première parte: la fonction $\zeta(s)$ de Riemann et les nombres premièrs en générale // Ann. Sci. Bruxells. 1896. V. 20. P. 183-256.

[3] Дэвенпорт Г. Мултипликативная теория чисел. М.: Наука, 1971.

[4] de la Vallée Poussin Ch. J. Sur la fonction $\zeta(s)$ de Riemann et les nombres des nombres premièrs inferieurs à une limite donee // Memories couronnes de 1' Acad. Roy. des. de Belgique. 1899-1900. V. 59. № 1.

[5] Виноградов И. М. Новая оценка $\zeta(1+i t) / /$ Изв. АН СССР. Сер. матем. 1958. Т. 22. № 1. С. 161-164.

[6] Коробов Н. М. О нулях функции $\zeta(s)$ // ДАН СССР. 1958. Т. 118. С. 431-432.

[7] Коробов Н. М. Оценки тригонометрическихх сумм и их приложения // УМН. 1958. T. 13. № 4. C. $185-192$.

[8] Walfisz A. Weylsche Exponentialsummen in der neuren Zahlentheorie. Berlin: Verlag der Wissenschaften, 1963.

[9] Arkhipov G., Buriev K. Refinement of estimates for the Riemann zeta-function in a neighbourhood of the line Re $s=1$ // Integral Transformation and Special Functions. 1993. V. 1. P. 1-7.

[10] Воронин С. М., Карацуба А. А. Дзета-функция Римана. М.: Физматлит, 1994.

[11] Карацуба А. А. Распределение простых чисел // УМН. 1990. Т. 45. № 5. С. 81-140.

[12] Schmidt E. Über die Anzahl der Primzahlen unter gegebener Grenze // Math. Ann. 1903. V. 57. P. 195-204.

[13] Littlewood J. E. Sur la distribution des nombres premiers // Contes Rendus. 1914. V. 158. P. 1869-1872.

[14] Лаврик А.Ф. Распределение простых чисел // Математическая энциклопедия. Т. 4. М.: Советская энциклопедия, 1984. С. 876-883.

[15] Landau E. Vorlesungen Über Zahlentheorie. V. 2. Leipzig: Verlag von S. Hirzelin, 1927.

[16] Ингам А. Е. Распределение простых чисел. М.-Л.: ОНТИ, 1936.

[17] Cramer H. Some theorems concerning prime numbers // Arkiv för Matematik 1920. V. 15. № 5 .

[18] Cramer H. Ein Mittelwertsatz in der Primzahltheorie // Math. Zeitschrift. 1922. V. 12. P. $147-153$

[19] von Koch H. Sur la distribution de nombres premiers // Acta Math. 1901. V. 24. P. $159-182$

[20] Kaczorowski J. On sign-changes in the remainder-term of the prime-number formula // Acta Arith. 1984. V. 54. №4. P. 365-377.

[21] Титчмарш Е. К. Теория дзета-функции Римана. М.: ИЛ, 1953.

[22] Hardy G. H., Littlewood J. E. Some problems of partitio numerorum III. On the expression of a number as a sum of primes // Acta Math. 1923. V. 44. P. 1-70.

[23] Попов А. Ю, Стечкин С. Б. Асимптотическое распределение простых чисел в среднем // II-я международная конференция "Алгебраические, вероятностные, геометрические, комбинаторные и функциональные методы в теории чисел”. Тезисы докладов. Воронеж, 1995. C. 128.

[24] Popov A. Yu., Stechkin S. B. Almost periodic functions and the asymptotic distribution of prime numbers // East J. Approx. 1996. V. 2. № 2. P. 143-150.

[25] Попов А. Ю, Стечкин С. Б. О поведении разности $\psi(x)-x$ // III Международная конференция "Современные проблемы теории чисел и ее приложения". Тезисы докладов. Тула, 1996. С. 116.

[26] Шилов Г. Е., Гуревич Б. Л. Интеграл, мера и производная. М.: Наука, 1967.

[27] Лаврик А.Ф. Дзета-функция. // Математическая энциклопедия. Т. 2. М.: Советская энциклопедия, 1984. С. 112-119.

[28] Бари Н. К. Тригонометрические ряды. М.: ГТФМЛ, 1961. 
[29] Бредихина Е. А. Почти периодическая функция // Математическая энциклопедия. Т. 4. М.: Советская энциклопедия, 1984. С. 543-545.

[30] Bezicovitch A.S. Almost periodic functions. Cambridge: Cambridge Univ. Press, 1932.

[31] Arno S. The imaginary quadratic fields of the class number 4 // Acta. Arith. 1992. V. 60. № 4. P. 321-334.

[32] Зигмунд А. Тригонометрические ряды. Т. 1. М.: Мир, 1965.

[33] Евграфоов М. А. Асимптотические оценки и целые функции. М.: Наука, 1979.

[34] Прудников А. Ф., Брычков Ю. А., Маричев О. И. Интегралы и ряды. М.: Наука, 1981.

Математический институт

Поступила в редакцию им. В. А. Стеклова РАН; 12.09.1996

Московский государственный университет им. М. В. Ломоносова 